University of Louisville

ThinkIR: The University of Louisville's Institutional Repository

Electronic Theses and Dissertations

8-2008

\title{
Acculturation and school adapation of Somali Bantu refugee children.
}

Manbeena Sekhon

University of Louisville

Follow this and additional works at: https://ir.library.louisville.edu/etd

\section{Recommended Citation}

Sekhon, Manbeena, "Acculturation and school adapation of Somali Bantu refugee children." (2008). Electronic Theses and Dissertations. Paper 1296.

https://doi.org/10.18297/etd/1296

This Doctoral Dissertation is brought to you for free and open access by ThinkIR: The University of Louisville's Institutional Repository. It has been accepted for inclusion in Electronic Theses and Dissertations by an authorized administrator of ThinkIR: The University of Louisville's Institutional Repository. This title appears here courtesy of the author, who has retained all other copyrights. For more information, please contact thinkir@louisville.edu. 


\title{
ACCULTURATION AND SCHOOL ADAPTATION OF SOMALI BANTU REFUGEE CHILDREN
}

By

\section{Manbeena Sekhon}

B.A., Fergusson College, 2000

M.A., University of Dayton, 2003

\author{
A Dissertation \\ Submitted to the Faculty of the \\ Graduate School of the University of Louisville \\ In Partial Fulfillment of the Requirements \\ for the Degree of \\ Doctor of Philosophy \\ Department of Educational and Counseling Psychology \\ University of Louisville \\ Louisville, Kentucky
}

August 2008 
ACCULTURATION AND SCHOOL ADAPTATION OF SOMALI BANTU

REFUGEE CHILDREN

By

Manbeena Sekhon

B.A., Fergusson College, 2000

M.A., University of Dayton, 2003

A Dissertation Approved on

July 16, 2008

By the following Dissertation Committee:

Samuel C. Stringfield, Ph.D., Dissertation Co-Chair

Thomas G. Reio, Jr., Ph.D., Dissertation Co-Chair

Kathleen M. Kirby, Ed.D., Ph.D.

Kathleen Moritz Rudasill, Ph.D.

Daya Singh Sandhu, Ed.D., NCCC, LPCC 
(C) Copyright 2008 by Manbeena Sekhon

All rights reserved 


\section{ACKNOWLEDGEMENTS}

I believe I wouldn't be writing this note, had it not been for Dr. Samuel Stringfield's incredible support through these past two years of my graduate study. From being lost and worn out having to work on a topic of little interest to then researching an area now of great passion, he supported and guided me through the end and I remain in awe of his hard work and commitment to the students of our program. Not to mention, it was Dr. Stringfield who steered me toward the mentorship and guidance of yet another amazing faculty, Dr. Thomas Reio, whose enthusiasm and support for my research topic has been extremely motivating. I owe Dr. Reio, my co-chair, much gratitude for his limitless patience, incredible sense of humor, willingness to involve himself with the Somali Bantu community and to share his wisdom to help me through this research process. Mostly, I appreciate his willingness to co-chair my dissertation despite his relocation to Florida.

I knew I had much to learn from Dr. Rudasill just having worked for her one summer two years ago. Her guidance and availability at the times I much needed has been key in alleviating my anxiety as well as helping me move forward with my work when I was feeling stuck. I owe Dr. Kathleen Kirby gratitude not just for her support for my dissertation, but for my entire time at the University of Louisville. Her passion for students and her sensitivity and willingness to advocate for their needs sets her apart from many others. I remain 
very grateful I had her support and mentorship these past five years. Last, but not the least, Dr. Daya Singh Sandhu's extensive knowledge and scholarship in the area of multicultural issues was indispensable.

A few other persons deserve acknowledgement. My friend Danielle Oatley and Marti Kinny from JCPS ESL department have been incredibly helpful and I cannot thank them enough. Their assistance in helping me gather important qualitative and quantitative data as well as all the wisdom and knowledge they imparted in the area of refugee children has been extremely helpful. Also, I owe special thanks to Dr. Geetanjali Gulati, a dear friend and a mentor, for always helping me stay focused and seeing the light at the end of the tunnel and Dr. Lee Look for being an incredibly patient and supportive listener who has always had more faith in me and my abilities than I seem to have in myself. Lastly, I am grateful for my internship site, SCS, Miami University, for allowing me the time off from work and at work to write my dissertation. I owe Dr. Pamela Wilkins and Dr. Maria Carrubba-Whetstine a big thank you.

This acknowledgement cannot be complete without my thanking Waheguru for his blessings and mostly thanking my amazing family. I owe all my success and learning to my parents as they have done everything and more to get me to where I am today. Lastly, having an incredibly supportive and caring brother and sister-in-law has made life away from home tenfold easier. I feel blessed 


\section{ABSTRACT \\ ACCULTURATION AND SCHOOL ADAPTATION OF SOMALI BANTU \\ REFUGEE CHILDREN \\ Manbeena Sekhon \\ July 16,2008}

The number of refugees being uprooted from their homes to seek refuge and resettlement in countries like the United States continues to grow, with large numbers being children. While each refugee group has its own set of challenges when adjusting to a new country, a better understanding of their needs may help facilitate their transition more quickly with lesser challenges. This study investigated the adjustment of one such group - Somali Bantus in a Midwestern U.S. city. Specifically, acculturation and school adaptation of Somali Bantu refugee children was explored using the Differentiated Multidimensional Model of Acculturation.

Somali Bantu refugee children in 5 elementary schools were asked to complete a questionnaire that assessed their level of acculturation to American culture and level of retention of Somali Bantu culture, at three levels i.e. language, identity, and behavior. The 5 ESL teachers were asked to complete a questionnaire that assessed total difficulties in behaviors for each of their Somali Bantu student. Correlations, MANOVAs, and hierarchical regression analyses were used to analyze the data. 
The findings of this study documented the significant role of school context in the acculturation and school adaptation of these Somali Bantu refugee children. The findings demonstrated that age was statistically, significantly, and negatively related to overall American Acculturation (AAI) and statistically, significantly, and positively related to Proficiency Level Composite scores. These students' AAI scores were statistically, significantly, and positively related to their Somali Acculturation Index (SAI) scores. Furthermore, SAl scores were statistically, significantly, and positively related to grades on Social Behavior and grades on Social Behavior and Work Study Habits were statistically, significantly, and positively related. Also, age and length of time in the country were statistically, significantly, and positively related.

The hierarchical regression analyses for $\mathrm{AAl}$ and $\mathrm{ABA}$ suggested that the demographic variables age, length of time in the US and gender did not contribute to any significant variance and school contributed a moderate variance to the overall $A A I$ and $A B A$ scores. School contributed substantially more incremental variance to ALA and AIA than did the other variables (age, length of time in the US and gender). Overall, the theoretical model including age, length of time in the US, gender and school explained 18,25,17 and 13 percent of the variance in the AAI, ALA, AIA and ABA respectively.

The hierarchical regression analyses for SAI, SLA, SIA and SBA suggested that the demographic variables age, length of time in the US and gender contributed marginally significant variance. School contributed substantially more incremental variance to SAI, SLA and SBA and a small 
incremental variance to SIA than did the other demographic variables. Overall, the theoretical model including age, length of time in the US, gender and school explained $36,20,15$, and 41 percent of the variance in the SAI, SLA, SIA, and SBA respectively.

The hierarchical regression analyses for the school outcomes suggested that the demographic variables age, length of time in the US and gender contributed marginally significant variance in Total Difficulties and Proficiency Level Composite scores but none for Grades on Social behavior and Work Study Habits. School contributed slightly more incremental variance to Total Difficulties and Proficiency Level Composite scores a. Acculturation did not contribute to any significant variance in any of the school outcomes. Overall, the theoretical model including age, length of time in the US, gender, school, and American acculturation explained $26,18,16$, and 38 percent of the variance and age, length of time in the US, gender, school, and Somali acculturation explained 26, 22,16 , and 41 percent of the variance in the Total Difficulties, Grades on Social Behavior, Grades on Work Study Habits, and Proficiency Level Composite scores, respectively.

The potential implications for theory and practice related to acculturation and school adaptation of Somali Bantu refugee children and teachers and school administrators working with them are provided. 


\section{TABLE OF CONTENTS}

PAGE

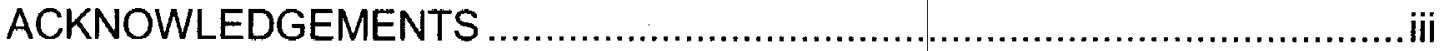

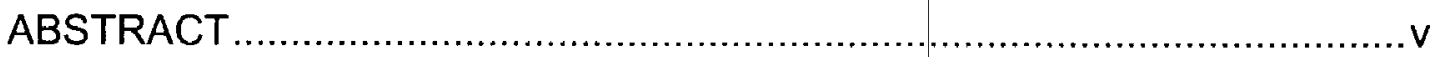

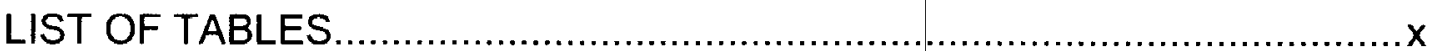

\section{CHAPTER}

I. INTRODUCTION

1

A. Importance of the Study in General

B. Role of School and Education in Refugee Children's Adjustment

C. Barriers to Adjustment and Success in Schools

D. Who are Refugees?

E. Types of Refugees

$F$. Refugees versus Immigrants

G. Refugee Children

H. Refugee Children's Needs for Success in the U.S.

I. Role of School/ Education in Refugee Children's Wellbeing

J. The Somali Bantus

K. Problem Statement

L. Purpose of the Study

M. Research questions

N. Operational Definitions

O. Significance of the Study

II. REVIEW OF THE LITERATURE

A. Acculturation

i. Theories of Acculturation

ii. The Differentiated Multidimensional Model of Acculturation

iii. Acculturation Studies

B. Refugee and Immigrant School Outcomes

i. Academic Achievement

ii. Refugee Youth in Resettlement

iii. Factors Related to Refugee/ Immigrant Children's School Outcomes 
iv. The School Experiences of Somali Refugee Youth in the U.S.

III. METHODOLOGY
A. Statement of Purpose
B. Selection of Participants and Procedure
C. Instrumentation
D. Data Collection
E. Research Design and Data Analyses

IV. RESULTS
A. Description of the Measures
B. Demographic Variables
C. Inter-correlations among Demographic Variables
D. Comparison of Sample Demographics
E. Contributors to Acculturation
F. Contributors to School Outcomes
G. Qualitative Analyses
$H$. Summary

V. DISCUSSION AND CONCLUSIONS

A. Discussion of the Main Results

i. Contributors to American Acculturation

ii. Contributors to Somali Acculturation

iii. Contributors to School Outcomes

B. Implications for Practice and Future Research

C. Limitations

D. Conclusion

REFERNCES

APPENDICES

A. Human Studies Approval

B. Language Identity Behavioral Acculturation Scale (LIB)

C. Strengths and Difficulties Questionnaire (SDQ) 


\section{LIST OF TABLES}

TABLE

PAGE

1. Demographic Information

2. Means and Standard Deviations for Acculturation and School

Outcome Variables

3. Inter-Correlations of Demographic, Acculturation, and School

Outcome Variables

4. Hierarchical Regression Analyses of Demographic Variables on

Composite American Acculturation (AAl)

5. Hierarchical Regression Analyses of Demographic Variables on

Composite Somali Acculturation (SAI).... 108

6. Hierarchical Regression Analyses to Predict School Outcomes Using Language, Identity, and Behavioral Dimensions of American Acculturation

7. Hierarchical Regression Analyses to Predict School Outcomes Using Language, Identity, and Behavioral Dimensions of Somali

Acculturation 


\section{CHAPTER I \\ INTRODUCTION}

The goal of the present study is to examine the relationship between acculturation and the different indicators of school adaptation among Somali Bantu elementary school-aged children. It seeks to assess the acculturation process of these Somali Bantu refugee children by looking at the different aspects of both their adaptation to American culture as well as the retention of their Somali Bantu culture. The study borrows Birman \& Trickett's (2001) multidimensional framework to understand acculturation to include three distinct domains - language competence, behavioral acculturation, and cultural identity (Birman, 1994; Birman \& Trickett, 2001; Birman \& Tyler, 1994; Trickett \& Birman, 2005).

The research seeks to answer the following questions: (a) To what extent do Somali Bantu children experience American acculturation at the three levels of acculturation, i.e., language, identity, and behavior, when considering demographic variables like age, years in the United States, gender, and school? (b) To what extent do the Somali Bantu children retain their Somali culture at the three levels of acculturation (language, identity and behavior) when considering demographic variables like age, years in the United States, gender, and school? and (c) To what extent does the contribution of American acculturation to school 
adaptation differ from contribution of retaining Somali culture to school adaptation (school grades on social behavior and work study habits, total difficulties on the Strengths and Difficulties Questionnaire, and Proficiency Level Composite score for English Language Proficiency on the ACCESS for ELLs) based on various demographic variables like age, years in the United States, gender, and school?

Chapter 1 focuses on the rationale for the study. It begins with a discussion on refugees in general including prevalence rates and the significance of research in the area of refugee children. This follows with a discussion on Somali Bantus and the importance in studying this particular group. Finally, Chapter I concludes with a summary of the significance for research with this refugee group.

In a 2006 report by the United Nations High Commissioner for Refugees (UNHCR) on refugee trends, refugee statistics in 95 countries worldwide total 6.2 million. The United States continues to act as a global leader in offering resettlement to refugees in urgent need of durable solutions. As per the 2005 Statistics Issue on the 2005-2006 U.S. Refugee program in the recent Refugee Reports, in the fiscal year 2005, the U.S. Refugee Program resettled about 53,800 refugees. The diversity of the refugees admitted to the U.S. in 2005 increased to 55 nationalities. O'Hara (2006) notes this is a significant shift from previous decades when refugees came primarily from Vietnam, the former Soviet Union, and the former Yugoslavia. This shift has presented resettlement agencies and communities welcoming refugees with a changing and increasingly complex client group requiring diverse culturally and linguistically appropriate 
services. Further, refugee arrivals from Africa represented the largest number of regional arrivals in the United States in 2005. They were 20,700 refugees of the total 21,000 proposed ceiling as set by the U.S, government for that year. Of these 20,700 refugees from Africa alone, about half were Somali refugees (O'Hara, 2006).

Given the large influx of refugees to the United States, increased attention is being focused on the adaptation of these populations to life in a new country (Trickett \& Birman, 2005). In looking at refugee children's adjustment, studies have noted the importance of school as the primary setting where refugee children first confront the "majority American culture" including language acquisition and cultural learning through experiences such as peer relations, classroom expectations, extracurricular activities and school norms (Boyd \& Shouse, 1997; Sarason, 1972; Trickett \& Birman, 2005). A review of the literature on immigrant and refugee school adaptation suggests that language, behavior, and identity are critical and independent acculturative domains in the school lives of these children (Trickett \& Birman, 2005). A group of refugee children in need of such research are the Somali Bantus. Despite the recent surge in resettlement of Somali Bantus into the United States, research on the acculturation and school adjustment of these children has been limited.

Importance of the Study in General

The research literature has linked refugee children's experiences related to stress and trauma associated with migration, displacement and acculturation in the resettled country with alarmingly high rates of Post Traumatic Stress 
Disorder (PTSD) symptoms they exhibit (Allwood, Bell-Dolan, \& Husain 2002; Birman, Trickett, \& Vinokurov, 2002). A 1998 study by Mollica, Pham, Fawzi, Murphy \& Lin found that $90 \%$ of the 51 refugees in their study met the criteria for PTSD. Lavik, Hauff, Skrondall and Solberg (1996) found that $48 \%$ of a refugee population met the criterion for PTSD. Similarly, Weine, Vojvoda, Becker, McGlashan, Hodzic, Laub and colleagues (1998) reported an incidence rate of $74 \%$ for PTSD in a Bosnian refugee population. However, as noted in the recent Review of Child and Adolescent Refugee Mental Health (2003) by the National Center for Child Traumatic Stress, very few of these children receive psychological services because of the stigma associated with seeking mental health care and a lack of understanding of the U.S. mental health system. The review further states that because of the complexities involved in addressing issues of trauma, culture, and ongoing stress within a developmental context, existing models of the etiology and treatment of a disorder like PTSD are often insufficient to meet the needs of these children. The report emphasizes the significant need for a culturally relevant, developmental and theoretical foundation to understanding the experience of refugee youth in the context of flight and resettlement. The latter is especially relevant in light of today's migration patterns having shifted in ways that bring new challenges to the field of refugee mental health. Lastly, it also notes that the new refugee arrivals are extremely diverse, with the largest place of origin today being Africa (Lustig, KiaKeating, Grant-Knight, Geitman, Ellis, Birman, Kinzie, Keane \& Saxe, 2003). Role of School and Education in Refugee Children's Adjustment 
Sinclair (2001) conducted a survey of education designed for emergency situations centering on refugee children before their resettlement to the United States (and other countries). She argued that education should be viewed as an essential element of humanitarian response to crisis. Sinclair adds that although funders often view education as a luxury in comparison to the essential needs of water, food, and shelter, when she reviewed reports of psychosocial trauma in young children she concluded that early educational responses support emotional and social healing as they help in restoring a sense of normalcy and hope. An evaluation of the Rapid Reponse Education Program that was developed to help children from Freetown, Sierra Leone deal with the aftermath of the 1999 humanitarian crisis, indicated evidence of improvement in just two weeks after program implementation. Sinclair found that recurrent pictures of traumatic events were reduced by $8 \%$, sleep difficulties declined by $49 \%$, and more than half of the children interviewed reported a sense of relief when they drew pictures, wrote, or talked about their war experiences. Thus, McBrien (2005) notes, that even though Sinclair's research was centered on education prior to the refugee children's resettlement in the United States, it revealed that by meeting the psychological and social needs of stressed and traumatized children through education, greater success in overall adjustment may be achieved. As noted previously, the school represents the primary setting where refugee children first confront the majority American culture, (Boyd \& Shouse, 1997; Sarason, 1972; Waters \& Leblanc, 2005). Further, as Delgado-Gaitan (1994), Trickett and Birman (2005), and Vinukurov, Trickett and Birman (2002) 
note, schools serve as the broad context for acculturation not only for academic development and language acquisition but also for cultural learning. They also note that schools are the locus of cultural conflict and acculturative stress hypothesized to be linked to maladaptive behaviors. Barriers to Adjustment and Success in Schools

Sinclair (2001) states that the trauma experienced by refugee children can impede their ability to learn. McBrien (2005) notes that toddlers, relocated in their rapid developmental period of rapid language acquisition and cultural socialization, are prone to language-related learning problems and social confusion. Further, trauma experienced during flight, in refugee camps, and during resettlement cause many refugees to become distrustful of people in authority (Hynes, 2003), and for children this group may could include teachers (Igoa, 1995).

Unfortunately, many researchers have found that when teachers have not been sufficiently trained to understand the difficulties and experiences of refugee children, they often misinterpret the students and their families' culturally inappropriate attempts to succeed in their new environment (Hones, 2002; Lee, 2002; Trueba, Jacobs, \& Kurton, 1990). McBrien (2005) notes that at present many teachers are frequently unaware that they have refugees in their classrooms, and that they do not realize the kinds of experiences that these students have survived before their arrival in the United States. Lastly, Hones (2002) found that teachers became more compassionate and willing to work with refugee and immigrant students when they became knowledgeable about their 
backgrounds. Thus, the school context along with teacher training and awareness of refugee children's traumatic past play a significant role in the teachers' experience of these children and their interactions with them, a lack of which could pose a serious threat to their adjustment. Who are Refugees?

Statistics/Prevalence Rates: Who are refugees? Since 1975, the United States has permanently resettled more than 2 million refugees (Cutts, 2000). McBrien (2005) notes that although admissions to the United States dropped sharply after September 11, 2001 they have been slowly increasing, with just under 53,000 refugees admitted in Fiscal Year 2004 (US Department of State, 2004). In the 1980 s, $85 \%$ of the refugees came from Latin America, the Caribbean, and Asia, with the Latin Americans constituting the largest number (Jensen \& Chitose, 1994). Since the 1990s, the refugees to the United States have fled war-torn Third World countries such as Afghanistan, Bosnia, Iraq, Burundi, Sudan, and Somalia, (U.S. Committee for Refugees and Immigrants, 2004). The statistics in The Yearbook of Immigration Statistics (Office of Immigration Statistics, 2004), suggest that some of the most approved refugee applications in Fiscal Year 2002 came from Bosnia-Herzegovina $(3,874)$, Cuba $(2,534)$, Iran (2,000), Afghanistan $(1,635)$, Ukraine $(1,618)$, and Sudan $(1,054)$. It also notes that the majority of applicants for refugee status for 2002 were from Somalia $(24,458)$, Ethiopia $(14,585)$, and Liberia $(13,283)$.

As per the United Nations definition used by the 1951 Geneva Convention relating to the Status of Refugees and its 1967 Protocol, a refugee is a person 
"who, owing to well-founded fear of being persecuted for reasons of race, religion, nationality, membership in a particular social group or political opinion, is outside the country of his nationality and is unable or, owing to such fear, is unwilling to avail himself of the protection of that country; or who, not having a nationality and being outside the country of his former habitual residence, is unable or, owing to such fear, is unwilling to return to it' (Convention and Protocol, 1951/1996).

People who flee their countries out of fear of persecution can apply for "Convention refuge" status, although whenever possible, the UNHCR's strongly prefers voluntary repatriation. However, political and civil turmoil in many countries may endure indefinitely and in these cases, the UNHCR seeks to settle refugees in their country of first asylum, i.e., the country to which they fled. The last solution chosen is permanent resettlement in a third country. Refugees may live in camps for years before they receive notice that a country is willing to resettle them (Hones \& Cha, 1999; McBrien, 2005; Tollefson, 1989). Further, other than in case of special circumstances, refugees are not able to choose the new country for resettlement (Cowart \& Cowart, 2002). Many refugees are poor and have few possessions to bring to their new lives (Caplan, Choy, \& Whitmore, 1994; Zhou, 2001). Finally, as McBrien (2005) and Nicassio (1983) note, those refugees with deficient language skills in their new country often suffer from high levels of alienation.

Types of Refugees 
The literature suggests that refugee groups differ from each other in various ways and it is important to understand these differences given the implications in how these different groups do when in a host country. To better understand the different types of refugees, work has been done in studying the various refugee movements over the decade. This work has led to distinguish between two types of refugee movements - anticipatory refugee movements and acute refugee movements.

McBrien (2005) and Kunz (1973) distinguish between anticipatory refugee movements, in which refugees foresee upcoming strife in their homeland, and acute refugee movements, in which danger is immediate. Anticipatory refugees have more time to plan their departure and may be able to learn some of the language of the country to which they will flee. Like voluntary immigrants, refugees in this group tend to be educated and financially solvent (Rumbaut \& Portes, 2001). The first wave of Vietnamese refugees, who arrived in the mid1970s immediately after the fall of Saigon, is an example of the anticipatory refugee movement. Most of them were well-educated professionals whose children excelled academically (McBrien, 2005). Their success contributed to the stereotype of Asians being the model minority group, i.e., the Asian model minority stereotype (Caplan, Whitmore \& Choy, 1989). Aside from their phenotype, this first group from Vietnam resembled what has been described as traditional refugees. Historically, traditional refugees have been represented by Eastern Europeans fleeing persecution during the Cold war. They tended to be more culturally and ethnically similar to those in the host countries, and many 
could locate kin who had migrated previously. They were also well educated and had skills that were valued by their host country. A similar group was the first wave of Cuban refugees who arrived in the early 1960s (McBrien, 2005; Perez, 2001, Stein, 1981).

Later refugees from Vietnam have been categorized as part of an acute refugee movement (Kunz, 1973). They arrived in greater numbers and were lacking education, job skills, and finances (Zhou, 2001). This second wave of refugees most of whom arrived from 1978 to the mid 1980s, became known as the "boat people." Those who did not die at sea arrived in Thailand, Malaysia, and Hong Kong, where they awaited resettlement. In addition, hundreds of thousands of Khmer fled the Pot Pot regime in Cambodia, and thousands of Laotians and Hmong escaped from the Pathet Lao. These refugees went to Thailand, and more than 300,000 were resettled in the United States (McBrien, 2005). Thus, depending upon the pre-migration circumstances and whether the refugees fall under the acute or the anticipatory movements, their adjustment outcomes differ significantly.

Refugees versus Immigrants:

In an attempt to better understand refugees and their experiences and adjustment in the country of resettlement, it is important to differentiate refugees from immigrants, given that many researchers tend to group refugees with immigrants. To gain an understanding of the unique needs of refugees, their obstacles to success and the interventions that are promising for them to overcome the barriers they face upon being resettled in the United States, it is 
critical to distinguish between refugees and immigrants as it is the differences between refugees and other immigrants that make this group the most vulnerable for failure (McBrien, 2005). But before getting into the differences, a brief discussion of similarities is presented as its these similarities that have led to the grouping of the two together.

Much research has grouped refugees together with immigrants (see Cheng, 1998; Cowart \& Cowart, 2002; Delgado-Gaitan, 1994; Hones \& Cha, 1999; Pryor, 2001; Rong \& Preissle, 1998; Portes \& Rumbaut, 2001). Researchers like Bozorgmehr and Sabagh (1991) suggest that refugees and immigrants have similar motivations and characteristics. Both groups have to deal with the disruption of migrating to a new country and adjusting to a different culture and lifestyle. Other researchers have noted that for students, this may include struggling in school while trying to learn the language of instruction. In addition, because of race, ethnicity, religion, or cultural differences, many newcomers, refugees or immigrants, encounter discrimination and racism (Asali, 2003; Rumbaut \& Portes, 2001; Suarez-Orozco \& Suarez-Orozco, 2001).

Further, both immigrant and refugee teens are faced with the additional crisis of identity as they try to meet the cultural demands of their parents and of their new peers (Delgado-Gaitan, 1994; Portes \& Rumbaut, 2001; Zhou \& Bankston, 2000). These similarities make it easy to have same associations for the two groups together.

Despite the similarities stated above, McBrien (2005) notes that given the United Nations Convention definition there are more important distinctions 
between refugees and voluntary immigrants. She notes that it is primarily the circumstances that bring refugees to a new country that create unique needs and problems that are not prevalent among voluntary immigrants. Voluntary immigrants choose to reside in a new country, and many are highly educated (Rong \& Preissle, 1998). They usually have time to think about their choice, and they may have visited the country of choice on past occasions (Cowart \& Cowart, 1993, 2002). Further, as Degado-Gaitan (1994) notes, many immigrants have sufficient financial means and are aware of family members, friends, or other people from their native country with whom they can settle in a community.

But, unlike most immigrants, refugees do not leave their homes by choice (UNHCR, 2000). Refugees are forced out of their native countries, often in violent circumstances such as civil war, and many homeless refugees must take up residence in temporary refugee camps (Cowart \& Cowart, 1993; Huyck \& Fields, 1981; McBrien, 2005). Living conditions in the camps are frequently poor, with inadequate food, shelter, and medical care (Westmeyer \& Wahmanholm, 1996). The living conditions contribute to significant, often chronic medical/ physical ailments, such as tuberculosis, hepatitis, malaria, kidney damage, and liver damage (Trueba et al., 1990). In addition, many refugees suffer from PTSD after enduring rape or torture and witnessing killings, often including the brutal murders of family members. Thus, it is the forced displacement and the circumstances leading to their resettlement that distinguishes refugees from immigrants (Hones \& Cha, 1999; McBrien, 2005). This clear yet critical distinction 
is helpful in identifying and understanding the particular barriers for more recent refugees, a discussion of which follows below.

Since the 1990s, most refugees from violence in Iraq, the Balkans, subSaharan Africa, and Afghanistan have belonged to the acute refugee category. People from these countries are unlikely to have high levels of education or vocational skills. McBrien (2005) notes that many of the recent refugees have two additional barriers they have to overcome. First, many are Black Africans with significant cultural differences from African Americans who are often perceived to be in the same cultural group by native-born White Americans. Secondly, many refugees from Africa and the Middle East are Islamic, a religious tradition many Americans have come to fear and despise and associate it with violence and terrorism (Asali, 2003; Carter, 1999; McBrien, 2005; McMurtrie, Bollag, Brender, del Castillo, Cheng, \& Overland, 2001, Wingfield \& Karaman, 2001). Thus, refugees from the recent acute movements have several challenges in the way of their post-migration adjustment. Further, given that this study is focused on Somali Bantu refugee children in particular, a discussion focusing on refugee children is essential and is presented below. Refugee Children

In 1994 the United Nations High Commissioner for Refugees (UNHCR) estimated that more than half of any refugee population is children (UNHCR, 1994). A more recent report stated that approximately $47 \%$ of the world's 19.2 million uprooted persons are youth under the age of eighteen (UNHCR, 2002a, 2005; Westmeyer, 1991). Further, the ratio of child to adult refugees varies 
depending on region as for example the majority of refugees in Central Africa are children (57\%) while only one-fifth of Central and Eastern European refugees are under eighteen (UNHCR, 2002a). Research suggests that surviving the war and its related trauma can have devastating social and psychological consequences for these youth (Wilkinson, 2002). During the 1990s, "four to five million (children and youth) were disabled, twelve million were left homeless and one million were orphaned or separated from their parents" (Summerfield, 1999, p. 111). Thus, given that a large number of refugees are children, it is essential to understand their developmental/ age specific needs, since adults and children differ in regard to their needs and stresses.

Children experience numerous traumatic situations when their lives are disrupted by the refugee experience (McBrien, 2005). They may have been exposed to many stressors related to war violence ranging from separation from caregivers to severe deprivation of basic needs, and from witnessing violence or death to experiencing torture (Boothby, 1994; Harrell-Bond, 2000; Kia-Keating, 2006; Pynoos, Kinzie, \& Gordon, 2001). Some refugee children suffer from family separation as they flee persecution alone or become separated from their families during flight (Boyden, deBerry, Feeny, \& Hart, 2002). Refugee children are at high risk for rape, abduction, and trafficking. Some children are forced to become child soldiers. Many girls become child brides. Refugee children become heads of households when parents or other adult caretakers are killed or die because of illness or malnutrition. As a result of these experiences/ 
circumstances, these children lose social stability and access to education (Boyden, Berry, Feeny, \& Hart, 2002; Tollefson, 1989).

The consequences of such severe, multiple and prolonged experiences of war adversities can profoundly impact refugee youths' mental health, leading to symptoms of PTSD, anxiety, and/ or depression (Allwood, Bell-Dolan \& Hussain, 2002; Kia-Keating, 2006; Kinzie, Boehnlein, \& Sack, 1998; Macksoud \& Aber, 1996; Sack, Him, \& Dickason, 1999). Further, Sack et al., (1999) state these symptoms can persist years after resettlement. Therefore, the refugee child's pre-migration experience often dictates their post-migration experiences in the country of resettlement. With this in mind, a more specific review of refugee children's needs for success in the country of resettlement is presented. Refugee Children's Needs for Success in the US

In her review of the needs of refugee students, McBrien (2005) found two predominant themes. The first is psychosocial wellbeing that includes a sense of safety, sense of self, and an adjustment to the cultural expectations of a new country while maintaining a connection to their heritage. Language acquisition is the second theme. As previously noted school is the primary agent for acculturation for refugee children and has been identified as key in helping refugee children develop psycho-social wellbeing and language acquisition. This makes the role of school and education in refugee children's success a critical area of investigation.

Role of School/ Education in Refugee Children's Wellbeing 
Given the continuing social upheaval in countries like Iran, Iraq, Afghanistan, Sudan, Somalia, and Sierra Leone, the demand for resettlement in host countries remains high (McBrien, 2005). Because over fifty percent of the refugee population is children, and that the Article 22 of the Geneva Convention requires states to provide equal schooling opportunities to refugee children, education must rank high on the list of provisions required for the successful relocation of these children. The same is required as per the Article 13 of the International Covenant on Economic, Social, and Cultural Rights, to which the United States is a signatory. Similarly, the UNHCR states that education is not only a fundamental human right but also an essential component of refugee children's rehabilitation (UNHCR, 2000). Research has indicated that education is crucial for restoring social and emotional healing (Sinclair, 2001) and that education and the school environment are key factors in facilitating socialization and acculturation of refugee and immigrant children (Hones \& Cha, 1999; Trueba, Jacobs, \& Kirton, 1990).

As previously noted, refugee children's post migration adjustment may vary depending upon various factors like the group/ nationality they belong to. Given these differences, a further discussion on the Somali Bantus and their history and background is essential in understanding their specific adversities that may define their post migration experiences.

\section{The Somali Bantus}

Who are the Somali Bantus? Since 1974, about 2.4 million refugees have been permanently resettled in the United States. While a large number of the 
early refugees were from Southeast Asia, current resettlement trends reveal a predominance of Middle Eastern and African refugees, many of whom are Muslim (McBrien, 2003).

Most African countries south of the Sahara are largely populated with Bantu-speaking people. The Bantus include over 300 distinct groups, each of which has its own distinct language or dialect. The Kikuyu of Kenya, the Swahili, whose language is spoken throughout eastern Africa and the Zulu of South Africa, are among the most frequently recognized Bantu groups (Lynch, 2005). As noted by Chanoff (2002), Somali Bantus, many of whom have sought refuge for years in the small town of Dadaab situated 60 miles from Somalia in the corner of Kenya's north-eastern province, are living reminders of the once vast Indian Ocean slave trade. She notes that their history as a distinct group began around the turn of the $18^{\text {th }}$ century when their ancestors from Malawi, Tanzania, and Mozambique were captured by the Sultan of Zanzibar and other slave lords and sold into Somalia, becoming victims of the millennia of human trafficking in East Africa. Known to be hereditary farmers, these people eventually settled into Somalia's arable regions along the Juba River.

Of a population of approximately nine million, pre-civil war Somalia constituted roughly 900,000 Somali Bantus. Most of these Bantus were integrated into society, with some arriving thousands of years ago as migratory farmers who saw themselves as original Somalis. Other Bantu people were brought in by Italian and British colonial administrations who also added to the work force. In her report on the resettlement of Somali Bantus, Chanoff (2002) 
notes that all these Bantus were part of the fabric of Somali society and contributed to their country in political, religious, and artistic ways during and after Siad Barre's post independence regime of 1969 to 1991.

The Somali Bantus who have been resettled in the United States are different, in that they are the descendants of slaves and originate from one of six main tribes: Majino, Makua, Manyasa, Yao, Zalama, and Zigua and are collectively known as Mushungulis (a word with multiple implied meanings including worker, foreigner, and slave). Thus, their slave origin and their ethnic and cultural differences from native Somalis, always kept them as a marginalized minority that never integrated with other Bantu people before the Civil War. Very few of them found opportunity beyond subsistence farming, and discrimination and poverty prevented them from accessing schools, land ownership, and everyday rights.

The 1991 civil war in Somalia caused these people to suffer widespread massacre and rape, as a result of which thousands fled to Kenya alongside other Bantu and ethnic Somali refugees. While the non-Mushunguli Bantus started returning to Somalia as early as 1993 , the Mushungulis could not as the warring militias had possessed their farms along the Juba River in addition to their awareness of the inequity, menace, and death that awaited them in the land they had sojourned for two centuries. Thus, for over a decade, Dadaab, Kenya had been their home where too they found themselves among a Somali majority and again were subjected to discrimination and danger. In 1997, the UNHCR attempted to resettle the group to their ancestral homelands in collaboration with 
the Mozambique government, but owing to a change in government and a lack of resources the process was cancelled last minute. Finally, in 1999, the United States government formerly recognized the plight of this twice displaced group and pledged to take them in. The resettlement verification process took a few years following which many Somali Bantus have been resettled in the United States since 2001 (Bradbury, 1997; Chanoff, 2002; Lynch, 2005).

The UNHCR officials document various important facts about the Somali Bantus,that in many ways differentiate them from most other prior refugee groups. A summary of some of those facts are presented in this section. As was noted by the Dadaab resettlement interviewers in Chanoff's (2002) report on the U.S. resettlement of Somali Bantu refugees, the Somali Bantus were not familiar with the use of dates and time. This was highlighted in their responses during their resettlement interviews with the UNHCR officials. Many women did not know their age, the age of their children, the time when they arrived in Dadaab or the town names along their route of flight from Somalia to Kenya. Prior to coming to the US, most Bantus had never had amenities like a refrigerator, stove, running water or kitchen cabinets. They had never lived with electricity, used a flush toilet, or been exposed to any other aspect of modern living. Age 16 is the typical marriage age for Bantu girls and around this time these girls could start bearing their own children.

Charnoff's (2002) report also discusses some gender role differences in that the boys more often than girls have been to school in Dadaab; they have mostly attended primary or secondary education. The girls rarely have time for 
school given the chores they have around the household. While some of the adults have finished high school, the majority of them have never attended school and as Charnoff (2002) notes that prior to coming to the US holding a pen between their fingers was an unknown awkward sensation. Lastly, a majority of these adults have never read or written in their own language. Many of the adults state that the lack of education is what kept the Mushungulis in subsistence, poverty-stricken lives as they never had a chance for education in Somalia.

Of the total number of Somali Bantus that were resettled in the U.S., over $60 \%$ were 17 years old or younger. One of every four Somali Bantu women gave birth in 2002. The average family has about four to six children. These Somali Bantus have lived for ten years on World Food Program rations of maize, beans, lentils, oil, flour and salt, leading to the concern that they would not recognize 99 $\%$ of the food in American supermarkets upon their initial arrival into the US. Approximately $40 \%$ of this group has never lived anywhere other than the refugee camp in Dadaab. While Somali Bantus made up only about ten percent of about 130,000 refugees in Dadaab, they still held over $90 \%$ of the heavy labor, construction, cooking, cleaning, and other manual labor jobs. Although most Somali Bantus are Muslim, a small minority converted to Christianity in Dadaab. While there are six main Somali Bantu tribes (Magindo, Makua, Manyasa, Yao, Zalamo, and Zigua), each tribe has many clans and sub-clans. Prior to coming to the U.S., less than $5 \%$ of Somali Bantus spoke English. Between 50 and $70 \%$ of Somali Bantus speak the Bantu version of the southern Somali dialect Maimai (also spelled Mamay, or Af Maay). Although 30 to $50 \%$ of Somali Bantu speak 
Somali, others understand Somali but prefer to speak in Maimai (Charnoff, 2002).

Lynch (2005) also discussed what Charnoff (2002) previously noted regarding literacy and the experiences of the Somali Bantus with regard to educational access and marginalization. They note that being denied access to education and having been prevented from learning to read and write their own language contributed to the low social and economic status of most Somali Bantu and that "this denial of access to education represents one of the most egregious and detrimental examples of Somali institutional discrimination against the Bantu" (Van Lehman \& Eno, 2003, p. 22). Given that far fewer schools were established in Bantu areas than in areas where dominant tribes lived, less than $5 \%$ of Somali Bantu have been formally educated (Charnoff, 2002; Lynch, 2005; Van Lehman \& Eno, 2003). Further, even if they did attend school, they were faced with instruction in an unfamiliar language. The Somali language has two distinct dialects that are similar in written form but not when spoken. As previously noted, Af Maay is most frequently spoken in southern Somalia where the Bantu live, while Af Maxaa is spoken throughout the rest of the country and in 1972, the government made Af Maxaa the official written language of the country, further keeping the Somali Bantu from participating in mainstream Somali society (Lynch, 2005).

Therefore, it is clear that the Somali Bantus bring with them a unique history of oppression that has shaped who they are as a result of the opportunities they had or a lack of, and which finally led to their being recognized 
as refugees by the United Nations. In shifting the focus from the Somali Bantus' pre-settlement experiences, a recent needs assessment study in one U.S. community in Maryland (Birman, Trickett, \& Bacchus, 2001) highlights some key aspects of their resettlement experience. The researchers found that the Somali community is very concentrated, with most of them living in one apartment complex; the population in the surrounding communities as well as the public schools is predominantly low income African American. As per the assessment (Birman, Trickett, \& Bacchus, 2001, p. 5), "the Somali children and youth reported feeling unsafe in their neighborhood," "particularly frightened by crime" as some of them have had dangerous confrontations. Further, "some described feeling worried that something may have happened to their friends when one of them is late and is walking home in the neighborhood." These youth "report seeing drug dealers in the neighborhood" and as the authors note, "these experiences rekindle the fear and uncertainty of their refugee experiences and make them feel like they are not yet safe."

With regard to the student experience, Birman et al. (2002) note that while many of the Somali students have formed relationships with American students over time, there are many difficulties linked to cultural differences around appropriate behavior particularly for adolescents. They find that Somalis who are friends with other Americans are restricted in many things that their American peers do given the restrictions on the part of their Somali culture. Such cultural differences affect the school experiences of these students. An example cited was that Somalis going out in mixed-sex groups is deemed inappropriate, 
thereby eliminating them from many of the more common American activities.

Thus, in order "to avoid getting into trouble, Somalis prefer to go out with other Somalis or with their families to the movies or to go shopping" (Birman et al., 2002, p. 7).

An important note with regard to Birman et al.'s (2002) needs assessment is that it does not distinguish whether the study was based on the experiences of all the Somalis inclusive of the Somali Bantus, or just the ethnic Somalis, or just the Somali Bantus. Given the added historical experiences of oppression the Somali Bantus refugees compared to the rest of the ethnic Somali refugees, such information if valid for the Bantu community only further adds to the plight of their situation in their attempt to adjust to a new society/culture.

Charnoff (2002) notes that while refugees who share a similar lack of U.S. knowledge, literacy, and cultural understanding have resettled in the United States previously and do so fairly regularly, the Bantus are an exception given their en masse resettlement. Some of the struggles that the Somali Bantus brought coming into the U.S. were their low literacy and English levels, large families, no U.S. support system and a lack of exposure to technology and urban living. These families share many stories of horror associated with their process of seeking refuge including children witnessing the death and rape of family members, executions in one's houses, and girls being kept captive for years and routinely abused by soldiers (Birman et al., 2002). Despite these known struggles and the central issue of trauma that they bring to their lives in the United States, the Somali Bantus are also recognized as a highly resilient community given their 
survival skills and tenacity to endure the refugee experience of rape, murder and flight, ten years in Dadaab, and a background of slavery and oppression. As Charnoff (2002) emphasizes in the United States Committee for Refugees (para. 59 ) "these refugees are rural farmers who yearn for a safe and quiet life. The dichotomy of their innocence and experience sharpens the importance of helping them. They finally have a chance to find their niche and redefine themselves," Thus, while a number of Somali Bantus have left behind a life of overt oppression in Africa, they continue to face a new set of challenges as they create a life for themselves in America (Lynch, 2005).

\section{Problem Statement}

Given the continuous influx of refugees and immigrants to the United States, the adaptation of these populations to life in a new country has emerged as an important focus of immigrant and refugee mental health (Trickett \& Birman, 2005). This wave of immigration in the past quarter century has been accompanied with a dramatic increase in the study of the acculturative process for immigrants and refugees. This process has been commonly defined as cultural change resulting from intercultural contact between two or more cultural groups (Berry, Trimble, \& Olmedo, 1986; Phinney, 1990). An important and specific aspect of inquiry of this literature is the question of how differing patterns of acculturation relate to successful adaptation in the new country (Birman, Trickett, \& Buchanan, 2005; Gordon, 1964; Holtzman \& Bornemann, 1990; Portes \& Rumbaut, 1990). 
The current study seeks to explore the acculturation and school adaptation of Somali Bantu refugee children resettled in the United States. It adapts Birman and Trickett's (2001) multidimensional model of acculturation from a life domain perspective in defining the acculturation process. Birman et al., (2005) drew on the "orthogonal" model of acculturation emphasizing the independent assessment of acculturation of the individual to the new as well as the the retention of the old culture. This perspective underlies much of their work with the Soviet Jewish refugee population (Birman et al., 2002; Birman \& Trickett, 2001; Birman \& Tyler, 1994) and is used by other acculturation researchers with different groups (e.g. Cortes, Rogler, \& Malgady, 1994; Nguyen, Messe, \& Stollak, 1999; Oetting \& Beauvais, 1990). By assuming that this process occurs both with respect to the new culture and the culture of origin allows the researcher to examine the nature of the relationship between acculturation to the two cultures (Birman, et al., 2005).

Thus, Birman and Trickett's (2001) multidimensional model of acculturation views the process of acculturation as multidimensional, involving three distinct processes: language competence, identification, and behavioral participation (Birman \& Trickett, 2001). Language competence assesses an individual's capacity to communicate in the languages of the two different cultures (home or host culture). Identity refers to the extent to which individuals embrace membership in either of the two cultures (Phinney, 1990), while behavioral acculturation, or engaging in behaviors characteristic of one culture or another is seen as resulting less from the individual's capacities and preferences, 
such as language competence, than from the transaction of the individual with the environment since some communities provide more opportunities to participate in one or both cultures than others. Hence ,Birman and Trickett (2001) describe the overall acculturation process in terms of the above three discrete acculturative processes that may unfold in different combinations (e.g. Birman, 1994) across different populations and settings (e.g. Birman \& Trickett, 2001).

Birman et al. (2005) refer to adaptation as the process of coping with the varied demands of the social contexts of importance to individuals. They adopted a life domain perspective in their study of the adaptation of a group of refugee adolescents from the former Soviet Union. As per this perspective, lives are comprised of a range of varied settings or microsystems which must be negotiated (Swindle \& Moos, 1992). For adolescents the important domains include psychological well-being as well as families, schools, and peer groups comprised of both immigrants and non-immigrants (Birman et al., 2002). Further they note that each of the domains may reflect different kinds of adaptive requirements. As an example, they explain that the development of successful supportive relationships may be viewed as an adaptive outcome among peers or family where as at school, academic achievement may be regarded as one of the indicators of successful coping (Birman et al., 2005). Thus, in studying refugee and immigrant adaptation, it is important to identify and address various life domains and how they are impacting the acculturation process.

Of the empirical evidence that does exist in the area of immigrant and refugee mental health, very little of it examines the acculturation and adaptation 
of Somali refugees, especially in the context of elementary school aged children and their school adaptation. Further, nothing is known about the adaptation and adjustment of the Somali Bantu refugee children to life in the United States. Given that these children are an integral part of the communities in the United States and their early and successful acculturation and adaptation within the schools and other microsystems like families and peers impacts and predicts future psychological well being, academic and employment success, it is extremely important to investigate their acculturation and adaptation processes.

Thus far, only a limited number of studies have investigated the psychological well-being of Somali (Bantu) refugees. As Kia-Keating (2006) notes, these few studies have focused on unaccompanied minors (Rousseau et. al., 1998; Sourander, 1998), youth adults aged 18-25 years (Halcon, Roberston, Savik, Johnson, Spring, Butcher, Westermeyer, \& Jaranson, 2004; Whittaker, Hardy, Lewis, \& Buchan, 2005), and some on Somali adult refugees living in London (Bhui, Abdi, Abdi, Pereira, Dualeh, \& Colleagues, 2003; McCrone, Bhuui, Craig, \& Mohamud, 2005), Australia (McMichael, 2002), the United States (Stutters \& Ligon, 2001), and Ethiopia (Bhui et. al., 2003; McMichael, 2002; Stutters \& Ligon, 2001; Zarowsky, 2000). These investigations identified the hardships that Somali refugees often experienced during pre-migration, including shortage of food, poor health, separation from family members, enforced isolation, feeling close to death, and being in combat situation (Kia-Keating, 2006). Additionally, there has been only one study noted that focused specifically 
on Somali adolescent refugees who had resettled with their families (Kia-Keating, 2006).

Because a review of the literature suggest a varied picture of the acculturation process and its relationship to adaptation across the different immigrant and refugee groups and contexts with multiple explanations for the differences (Birman et al., 2005) and because there have been relatively few studies that have examined these in the context of Somalis, let alone the Somali Bantu refugees, an empirical study of this nature is warranted. Thus, the focus on refugee children who represent an understudied population presents a distinctive opportunity to extend the range of knowledge about the acculturation process and its relationship to adaptation, offering an additive framework for enlarging our understanding of cultural diversity. The present study is intended to contribute to this empirical literature by examining the relationship between acculturation and the different indicators of school adaptation among a group of elementary school aged Somali Bantu refugee children.

Purpose of the Study

The purpose of this study is to extend the research on acculturation and adaptation processes of refugees and immigrants by focusing on one highly specific refugee group - the Somali Bantu refugees in the United States. The study is designed to operationalize acculturation in a highly differentiated manner by adopting the "orthogonal" (i.e., statistically independent) multidimensional model of acculturation. The present study intends to test the relationship between acculturative styles and school adaptation among a group of Somali Bantu 
refugee children, using a multidimensional model of acculturation that includes language, identity and behavior. As per this model, acculturation for each child is measured independently with respect to both Somali Bantu and American cultures. As Birman and Trickett (2001) note, the three aspects of acculturation are relatively distinct from each other but as noted previously are also differentially related to various school outcomes.

Research Questions

The following research questions are used to explore the problem for this study on the relationship between the acculturation and school adaptation of Somali Bantu refugee children.

1. To what extent do Somali Bantu children experience American acculturation at the three levels of acculturation, i.e., language, identity, and behavior, when considering demographic variables like age, years in the United States, gender, and school?

2. To what extent do the Somali Bantu children retain their Somali culture at the three levels of acculturation (language, identity and behavior) when considering demographic variables like age, years in the United States, gender, and school?

3. To what extent does the contribution of American acculturation to school adaptation differ from contribution of retaining Somali culture to school adaptation (school grades on social behavior and work study habits, total difficulties on the Strengths and Difficulties Questionnaire, and Proficiency Level Composite score for English Language Proficiency on 
the ACCESS for ELLs) based on various demographic variables like age, years in the United States, gender, and school?

Operational Definitions

The following definitions are given to clarify terms used in this study to avoid misinterpretation.

Refugee - A refugee is a person who, owing to well-founded fear of being persecuted for reasons of race, religion, nationality, membership in a particular social group or political opinion, is outside the country of his nationality and is unable or, owing to such fear, is unwilling to avail himself of the protection of that country; or who, not having a nationality and being outside the country of his former habitual residence, is unable or, owing to such fear, is unwilling to return to it (United Nations Convention and Protocol, 1951/1996).

Acculturation - It is the process of cultural change that occurs as a result of contact between members of two or more cultural groups (Berry, 1980; Persky \& Birman, 2005).

Adaptation - It is the process of coping with the varied demands of the social contexts of importance to individuals (Birman et al., 2005). Multidimensional Model of Acculturation: It is a model that views the process of acculturation as multidimensional, involving three distinct processes: language competence, identification, and behavioral participation (Birman \& Trickett, 2001). Language competence assesses an individual's capacity to communicate in the languages of the two 
different cultures. Identity refers to the extent to which individual's individuals embrace membership in either of the two cultures (Phinney, 1990), while behavioral acculturation, or engaging in behaviors characteristic of one culture or another is seen as resulting less from the individual's capacities and preferences, such as language competence, than from the transaction of the individual with the environment since some communities provide more opportunities to participate in one or both cultures than others. Thus the overall acculturation process is described in terms of three discrete acculturative processes that may unfold in different combinations (e.g. Birman, 1994) across different populations and settings (e.g. Birman \& Trickett, 2001).

\section{Significance of the Study}

Recent research emphasizes the need for and benefit from further empirical research that aims at understanding the acculturation and adaptation of new immigrant and refugee populations within the context of different microsystems such as schools. Such an inquiry will enrich an appreciation of the complexities of the adaptation process of the immigrant and refugee populations by providing an increasingly sophisticated framework for those concerned about how and where to intervene in the life domains of importance to immigrant and refugee children (Birman et al., , 2005).

In her extensive review on the educational needs and barriers for refugee students in the United States, McBrien (2005) emphasizes the importance of research in understanding refugee children's acculturation in the school context 
and their school outcomes. She regards this knowledge as critical in providing background information necessary for teachers and counselors in the field. This knowledge is also significant for those in universities preparing new teachers to provide educational opportunities in a caring way to students. This research becomes even more important given very little is known about these refugee students especially since they are the ones who may require the greatest patience and teaching expertise.

Thus, the results of this study in examining the acculturation and school adaptation of a very specific and unexamined population, i.e., Somali Bantu refugee children may add significantly to the refugee mental health literature and increase our understanding of these Somali Bantu refugee students' needs and experiences in particular. With a better understanding of how these children are acculturating to their lives in the United States and adapting to their schools, teachers, counselors, and other community agencles that are involved in serving them, may help develop alternative ways to better meet their needs and assist them in being more successful academically as well as psychologically. A study of this nature will add to the empirical literature in immigrant and refugee mental health as well as facilitate the development of policies impacting this population and the refugee population in general. A study of this nature also benefits the educators training future teachers, social workers and mental health professionals, especially those educating and/or working with refugees and immigrants. Lastly, this research also hopes to further the field of cross cultural 
psychology through a deeper understanding of acculturation and its various aspects, i.e., language, behavior and identity in the context of Somali Bantus. 


\section{CHAPTER II}

\section{REVIEW OF LITERATURE}

Chapter Two provides a review of the salient literature relating to the acculturation and school adaptation of Somali Bantu refugee children resettled in the United States. As was discussed in chapter one, the empirical and theoretical literature on the acculturation and school adaptation of the Somali Bantu children is limited. Thus, chapter two draws from literature in the area of acculturation and school adaptation of the general immigrant and refugee population to extrapolate to the acculturation process of the Somali Bantu refugee children's post migration adjustment.

This review will begin with a general discussion on acculturation and the various associated theories with a focus on the Differentiated Multidimensional Model of Acculturation with an expanded discussion of behavioral, language, and identity acculturation. The discussion on acculturation will then shift to a review of the literature on immigrant and refugee children's school outcomes including academic achievement and psychological well-being and the various factors impacting these school outcomes. A brief discussion on protective factors of refugee youth in resettlement will follow. The chapter will conclude with a review of the only needs assessment study done on Somali youth in a US community. Acculturation 
As noted by Trickett and Birman (2005) and defined by Berry (1994) and Phinney (1990), the general set of processes involved in an immigrant or refugee's adaptation to a new country is called acculturation, "or the cultural change resulting from intercultural contact between two or more cultural groups" (p. 426) that leads to change in all parties especially in the acculturating group. McBrien (2005) defines acculturation as the change in an individual or a culturally similar group that results from contact with a different culture.

Berry, Poortinga, Segall, and Dasen (2002) point out that there are differences between psychological and sociological acculturation. At the individual level, changes can occur in one's sense of identity, values, and beliefs. People may experience acculturative stress, such as anxiety or depression, as they try to adapt to a new culture. At the group level, change affects social structure, economic factors, and political activity. As an example, McBrien (2005) notes that the Cubans were able to maintain their social structure and standard of living because of the reception they received when they began arriving in the United States in the late 1950 s and early 1960s. In contrast, many Nicaraguans, although highly educated, lost their ability to maintain professional jobs or to create an enclave because the U.S. government rejected their claim for asylum. Stein (1979) studied World War II refugees and Hungarian, Cuban and Vietnamese refugees. He noted that occupational and economic adjustment is crucial to adult refugees' acculturation in a new country and educational success is essential for refugee children's acculturation and that these group processes affect one's individual sense of identity and well-being. 
As noted above, the acculturation process of refugees and immigrants differ for different populations depending upon key factors including the individual, social, and political circumstances leading to the migration. On another note, the Review of Child and Adolescent Refugee Mental Health (2003) notes that the development of theoretical frameworks on refugee children's mental health frequently identify the chronological stages of a refugees' experiences, i.e., preflight, flight and resettlement, to organize the research and clinical work (Berman, 2001; Fox et al., 1994; Papadopoulos, 2001). Thus, different perspectives and factors have led to the development of various theories conceptualizing acculturation and adaptation of immigrants and refugees. The following section presents a summary of some of the key theories on acculturation.

Theories of Acculturation

Ogbu (1982) explains that adapting to a new culture is affected by whether one is a voluntary or an involuntary immigrant (such as a slave). He places refugees in a middle category, describing them as semi-voluntary immigrants. $\mathrm{He}$ notes that voluntary immigrants view learning the language and ways of the dominant culture as desirable avenues to success, whereas people with an oppositional cultural frame of reference such as colonized or enslaved people view conformity as a "symbol of disaffiliation" with their own culture. Thus, members of the oppositional cultures are more likely to reject the host culture, viewing separation and segregation as desirable goals. Voluntary immigrants, by contrast, tend to choose an additive acculturation strategy in which they acquire 
new cultural tools without rejecting their native knowledge and skills (Gibson, 1998). This strategy is termed accommodation and acculturation without assimilation, in which the individual can draw from more than one culture to accommodate a given social context (Gibson, 1998).

The acculturation literature includes many theories, some similar and other contrasting about the process of acculturation. Proponents of assimilation believe that acculturation is best when it involves assimilation, i.e., the process in which individuals give up their old culture, exchanging it for the culture of their new society (McBrien, 2005). But then there are advocates of cultural pluralism who believe that newcomers acculturate best if they are able to retain cherished values of their homeland while adding the language and some customs of their new home. Some other related terms in the literature include biculturalism, transculturalism, and additive assimilation, where the individual is able to move between cultures at will, depending on the situation (McBrien, 2005).

In his extensive review of acculturation theories, Rudmin (2003) pointed out that since 1918, some researchers have hypothesized that a bicultural identity is psychologically beneficial where as others argue such an identity is impossible or unhealthy. Rudmin criticized the lack of empirical evidence for these theories. His criticism included Berry's (1974) widely respected acculturation taxonomy, which includes assimilation (in which one takes on the majority culture and rejects the minority culture), separation (in which one clings to minority culture and rejects the majority culture), marginalization (in which 
neither culture is embraced) and integration (in which components of both cultures are embraced).

Portes and Zhou (1993) on the other hand, proposed a theory of segmented assimilation that argues that there are three possible patterns for immigrant adaptation into the new society, and that these patterns are dependent on social, political and economic factors. The first is described as the straight-line theory of upward mobility, in which newcomers assimilate into the White middleclass majority (Rumbaut, 1994). Irish immigrants arriving at the turn of the $20^{\text {th }}$ century and the post-World War II Eastern European refugees are examples (McBrien, 2005). The second pattern is that of upward mobility and ethnic solidarity, found in successful ethnic enclaves that have established themselves through supportive governmental and social policies. Some Vietnamese and Cuban communities in the United States fall into this category. This pattern is comparable to what other researchers call additive assimilation. The third pattern of acculturation is described as more of an unsuccessful downward spiral process resulting in assimilation into poverty with cultural dissonance. In this process the children tend to acquire the language and skills of their new culture more quickly than their parents do, resulting in family conflicts. Gibson (2001) notes that for these children, the acculturation process is subtractive, i.e., to identify with being American; the children join oppositional subcultures of marginalized peers and reject the culture of their parents. McBrien (2005) notes that this tends to be the fate of many families that the United States refuses to recognize as legitimate immigrants, and who often end up settling in depressed 
urban areas with high rates of crime and unemployment. Portes and Zhou (1993) explain that many refugees arriving since the late 1970 s are less likely to blend into White society than their predecessors, because of their racial and ethnic origins. Thus, without significant social support, recent refugee children are especially vulnerable to such a negative pattern. This theory appears to be in line with the previously discussed literature that emphasizes the role of pre-migration factors on acculturation.

Portes and Rumbaut (2001) elaborated on segmented assimilation theory with their analysis of the Children of Immigrants Longitudinal Study, a 10-year project in which the researchers gathered data from more than 5,200 youth enrolled in 49 schools in Fort Lauderdale/ Miami and San Diego, whose parents came from 77 countries. The purpose of the study was to examine the adaptation processes of second generation immigrant youth which included voluntary immigrants and refugees. Portes and Rumbaut explained that segmented assimilation patterns depended on four factors: the situation of the first generation that immigrates; the pace at which parents and children acculturate; cultural and economic barriers confronted by immigrant youth; and resources (family and community) available to manage the barriers. They note that some recent refugees cannot melt into the dominant culture because of the stigma of their religious garb or their phenotype. Both Portes and Rumbaut (2001) and McBrien (2005) argue that governmental and societal policies that encourage or discourage welcoming refugees from various countries play a prominent role in their success in the host country and all of these factors and patterns ultimately 
have a significant effect on immigrant and refugee children's success to education and opportunity.

Nguyen, Messe, and Stollak (1999) limited the use of the term acculturation to cultural pluralism, or the additive model (i.e., when all groups within a larger society maintain their unique cultural identities) in their quantitative study of 182 junior and senior high school Vietnamese students. The researchers found a positive correlation between US cultural involvement and self-esteem, low depression, family relationships, and grade point average. They also found that Vietnamese cultural involvement corresponded positively with family involvement, not at all with school GPA, and negatively with personal adjustment. The findings were in contrast to the researchers' hypothesis that adaptation to American culture would negatively affect family relationships. The findings also conflicted with the idea that bicultural identification produced positive results, because involvement with Vietnamese culture was not positively correlated with personal adjustment.

McBrien (2005) draws from the segmented assimilation theory to explain the unexpected findings in Nguyen et al.'s (1999) study, suggesting that the adults and children in the study could have experienced congruence in their adaptation to a US lifestyle. Thus, their US cultural involvement would not affect their family relationships. She further extrapolates that that perhaps some youth held tightly to Vietnamese culture as a result of discrimination from US peers. Given the results of this study, both McBrien (2005) and Nguyen et al. (1999), conclude that acculturation a complex multidimensional process. 
In the current study I seek to understand the acculturation of the Somali Bantu refugee children based on the above conceptualization that acculturation is a multidimensional process. Building upon Trickett \& Birman's (2005) study using a differentiated multidimensional model of acculturation to assess the relationship between the acculturation and school adaptation of a group of refugee adolescents from the former Soviet Union, is also aimed at assessing the relationship of acculturative styles to school adaptation, but among a refugee group of elementary school children from Somalia. Before moving into the discussion of the literature on Birman and Trickett's differentiated multidimensional model of acculturation, readers should keep in mind that the studies in the following discussion involve diverse populations, thereby limiting the generalization that may be drawn from the findings.

The Differentiated Multidimensional Model of Acculturation

In their study on a group of 110 refugee adolescents from former Soviet Union, Birman and Trickett (2005) assessed the acculturation of those adolescents with respect to both American and Russian cultures and, within each culture, looked at language competence, behavior and identity. Thus, they examined how much Russian culture these young adolescents were retaining and how much American culture they were adopting with regard to language competence, behavior and identity.

Related to immigrant and refugee children's school adaptation, many researchers have noted that the school represents the primary setting where refugee children first confront the majority American culture, and that the school 
serves as a broad context for acculturation for academic development, language acquisition, and for cultural learning through experiences such as peer relations, classroom expectations, course offerings, extracurricular activities, and school norms around parental involvement (Boyd \& Shouse, 1997; Delgado-Gaitman, 1994; Sarason, 1972; Trickett \& Birman, 2005; Vinokurov, Trickett, \& Birman, 2002). Trickett and Birman (2005) also note that while the majority of the immigrant and refugee school adaptation literature does not include specific measures of acculturation, it does suggest that language, behavior, and identity are critical and differentiated acculturative domains in the school lives of these children. Thus, Birman, Trickett and their colleagues have employed the differentiated multidimensional model of acculturation in their several of their studies with Soviet Jewish refugees in the US, assessing acculturation in these refugees with respect to both the new and old cultures $(2006 ; 2005 ; 2002,2001$; 1998; 1994). The model is multidimensional given it addresses three distinct aspects of the acculturative process - language competence, cultural identification, and behavioral participation.

The differentiated multidimensional model of acculturation views behavioral acculturation as a process that is based on the interaction of an individual's competencies and preferences with opportunities to engage in cultural activities in the surrounding community. Birman (2006) elaborates this with an example that whether someone uses English when speaking to friends depends on whether the friends are able to speak English, whether the speaker wishes to interact with Americans, and whether there are opportunities to 
become friends with Americans in the community, concluding that behavioral acculturation reflects both individual attributes and contextual factors.

In this differentiated multidimensional model, language competence describes an individual's capacities and that although these capacities are constrained by context, they do not in and of themselves reflect such transactions (Birman, 2006; Zea, Asner-Se, Birman \& Buki, 2003). Birman (2006) notes that while other studies assess language acculturation through measures of language use or preference the multidimensional model conceptualizes language use as behavioral acculturation that relates not to competence but to situational demands. Since both language use and preference can only be achieved with language competence, the model assesses self-reports of language competence or the ability to speak and understand the language.

The third dimension of the differentiated multidimensional model of acculturation is cultural identity and is based on Phinney's (1990) work on cultural identity. He describes it as the extent to which individuals embrace membership in either of the two cultures and consists of two components - selfdesignation as a member of a group and positive affect toward this identity as a group member.

\section{Acculturation Studies}

Birman, Trickett and their colleagues have applied the differentiated multidimensional framework of acculturation to several of their studies on the acculturative process of Jewish adolescents from the former Soviet Union. The sections that follow discuss and summarize some of their seminal work and key 
findings on various acculturation and various outcomes using the multidimensional model.

In one of their studies, Birman \& Trickett (2001) looked at the extent to which life stage differences at the time of immigration affect the acculturation process of these adolescents and their parents. The authors found that overall acculturation for these Jewish Russian adolescents appeared to occur in a linear pattern over time for most dimensions of acculturation, with acculturation to American culture increasing and acculturation to Russian culture decreasing with time.

In another study of the same population, Trickett and Birman (2005) examined the relationship of acculturative styles to school adaptation among a group of 110 refugee adolescents from the former Soviet Union and their parents. School adaptation was measured in terms of academic (GPA), behavioral (disciplinary infractions), and attitudinal (sense of school belonging) components. As per the results, differing patterns of overall American and Russian acculturation were associated with differing school outcomes, as were language competence, behavior, and identity with respect to the different cultures. The authors noted that the level of American acculturation proved to be a generally positive predictor of school adaptation, while the overall level of Russian acculturation was unrelated to school outcomes. The results confirmed Trickett and Birman's (2005) prediction of the adaptive value of American acculturation in the school, as higher levels of American acculturation were 
related to each of the school outcomes in a positive way (better grades, fewer absences, and a greater sense of school belonging).

Trickett and Birman (2005) found that unlike much of the previous research that has found retention of the culture of origin to be a positive factor in school performance, in the present study, overall retention of Russian culture did not serve this function while overall American acculturation did. Some of the explanations presented by the authors were that there is a positive association in the Soviet Jewish community of American acculturation with academic and subsequent economic success; that for Soviet Jews, immigration to the United States allows them to join the White majority where as in the former Soviet Union they had been members of a minority group. This, they noted, is in a sense a reverse process from the experiences of Asian or Latin American immigrants, for whom migration to the United States means becoming ethnic/ racial minorities unlike their previous membership to the majority culture in their countries of origin. With regard to these differences in findings across populations, the authors emphasized that a complex and contextualized understanding of acculturation and ethnic identity could be reached only through studies of multiple racial and ethnic/ immigrant refugee groups living in varied kinds of school and community contexts. The authors concluded that in general, higher levels of American acculturation predicted school adaptation whereas aspects of Russian acculturation were differentially related to school adaptation for different subgroups for the Jewish Russian refugee adolescents. 
In exploring yet another aspect of acculturation employing their differentiated multidimensional model of acculturation, Birman, Trickett and Vinokurov (2002) sought to examine if and how acculturation is related to adaptation across different life spheres for 162 Soviet Jewish refugee adolescents in a suburban community in Maryland. The authors proposed that because the different contexts of refugee adolescents' lives vary in acculturative demands, different patterns of acculturation should be related to adaptation in different life spheres. Their study looked at acculturation to both American and Russian cultures in relation to their psychological adaptation, peer relations, and school and family outcomes. Again, the findings indicated that acculturation to different cultures is differentially related to adaptation across life domains. Acculturation to American culture predicted better grades and perceived support from Russian peers. Both American acculturation and retention of Russian culture predicted reduced loneliness and perceived support from parents. Hence, the findings of this study suggest that acculturation is a complex process that not only depends upon the context, but that it also plays out differently for different life domains like psychological adaptation, peer relations and school and family outcomes.

Birman et al. (2005) replicated the above study with the same Soviet Jewish refugee population in a contrasting community within the same state (Maryland) to explore the potential role of community differences in the acculturation and adaptation processes of 269 refugee and immigrant adolescents from the former Soviet Union who resettled in two different 
communities, concentrated (represented a geographically small area where former Soviet Union émigrés were concentrated in a few neighborhoods and schools just outside the city line) and dispersed (throughout an entire county where former Soviet Union émigrés were dispersed throughout the county's multicultural neighborhoods and schools). Adaptation was assessed across different life domains, including peer relationships, family relationships, school adaptation, and psychological adaptation. The length of residence by community interactions was found significant suggesting that the community context impacts how rapidly the adolescents acquired all three dimensions of American culture assessed in the study. As per the results, American acculturation seemed to occur more rapidly in the dispersed community with respect to language, identity and behavior compared to the concentrated community. But in contrast, the community context was less of an influence on the rate of loss of identification with and attachment to Russian culture. Thus as previously documented, the findings of these studies indicate that not only does acculturation differ based on different life domains, but that it also varies within the context of the different communities in which the participants reside.

Language Competence. In studying the acculturation process of Jewish adolescents and their parents and the extent to which life stage differences at immigration affect the acculturation process, Birman and Trickett (2001) found that over time the adolescents' ability to speak and understand English improved whereas their ability to speak and understand Russian declined. The authors noted that the ability to speak and understand English for the adolescents 
surpassed their ability to speak in Russian after living in the country for approximately 4 to 5 years. Further, the results indicated that the level of American language acculturation was significantly higher than the level of Russian language competence. On the other hand, they found that Russian language competence for the parents did not diminish with length of residence in the country although their English did improve. In fact the parents' Russian language competence was significantly higher than their English language competence. This highlighted the adolescent-adult differences in the acculturation process in the context of English language acquisition.

The authors concluded that for adolescents in their sample, English language acquisition represented a transformative experience because English language competence appeared to replace competence in Russian over time and the age of arrival assumed considerable importance in language acquisition and loss. On the other hand, it was found that for the parents, Russian language competence remained unchanged with length of residence, where as English language competence improved. The authors concluded that their data was suggestive of an adhesive or additive process of acculturation for the parents. Interestingly, Trickett and Birman's (2005) previously discussed study examining the relationship of acculturative styles to school adaptation among a group of 110 refugee adolescents from the former Soviet Union and their parents, the authors had predicted that English language competence would be predictor of school outcomes. The authors found that even though overall levels of American acculturation proved to be a generally positive predictor of school 
adaptation, expectation that English language competence would similarly predict school outcomes in these adolescents was not supported. This was an interesting finding given the common wisdom and prior research suggesting that English language skills are most important in helping immigrant students succeed in U.S. schools. Trickett and Birman explained that the adolescents in their study had graduated from ESL before coming to the school where the data was collected and thus their level of English competency may have crossed the "good enough" threshold such that language was not a critical factor in their academic achievement as may be the case for less English-proficient students. In conclusion, the findings suggest that English language proficiency may not be as much of a relevant variable in predicting school outcomes once student proficiency in English language has been attained.

In their study on how acculturation is related to adaptation across different life domains for a Jewish refugee population, Birman et al. (2002) concluded that the three dimensions of acculturation were found to be differentially related to the adolescents' adaptation. As was the case in the previous study, the authors had expected English language competence to be the most important predictor of school grades for these Jewish Russian adolescents. Again, contrary to what they predicted, Russian language competence and not English language competence was found to predict GPA, and the relationship was in the positive direction. The authors suggest that perhaps Russian language proficiency after several years of being in the United States is a marker for other achievementrelated attitudes or capabilities, and thus the finding related to greater Russian 
language competence predicting better GPA in these adolescents. The authors also discuss the possibility that students who are high achievers are likely to rate themselves better in many areas of functioning, and in this context, their Russian language competency. Another key finding in this study was the contribution of English language proficiency to reduced symptoms on the Hopkins Symptoms Checklist that for psychological adaptation. The authors concluded that perhaps English language competence is the cornerstone of positive personal adjustment for those adolescents as it allowed them the greatest flexibility and options in terms of involvement with school and the culture more broadly.

In their follow up study looking at the potential role community differences in the acculturation and adaptation process of Soviet Jewish refugees, Birman et al., (2005) found that in contrast to prior findings on adults living in different types of communities, language competence did not differ between residents of the concentrated and dispersed communities with respect to either English or Russian. The authors discussed that even in the concentrated community their sample was a language minority within a surrounding local community. Further, the ethnic enclave was not large enough leading to a considerable need to interact with the English speakers which may also be reflective of the developmental ease with which adolescents acquired English skills compared to adults. Lastly, the adolescents in the study came from relatively well-educated families where learning English was likely to be encouraged. The authors concluded that all these factors jointly contributed to the relatively rapid process of acculturation whereby the adolescents were losing their Russian language 
skills and the new language was becoming more dominant over time. Therefore, opportunities for language use and interaction within their immediate and community surroundings can change the nature of the acculturation process for individuals.

The literature discussed so far has focused on examining how various aspects like life domains and community contexts impact the language competence domain of the differentiated multidimensional model of acculturation. The following discussion reviews some of general the literature on language proficiency and various aspects of adaptation.

Bhattacharya (2000) explored risk and protective factors within contexts of family traditions, familial values, and social norms of south Asians to examine the school adjustment of 75 South Asian children (aged 6-17 years) who had immigrated to the US with their parents, and who had been referred for evaluation due to below-average or failing grades. Bhattacharya found that low English language proficiency was a critical factor in the low achievement and school failure of these children. Similarly, in his study examining the factors influencing immigrant students' school achievement on 4,288 eight - ninth graders belonging to nine different ethnic categories (Southeast Asian, other Asian, Jamaican, Haitian, Mexican, Cuban-public school, Cuban private-school, other Latin American, and Filipino), Portes (1999) found that amongt other factors, lowest achievers were from groups that encountered language problems in school; thus, also suggesting that school adaptation may be driven by language proficiency. In addition, in their study examining the effects of ethnicity, 
generational status, duration in the US, language usage, and social capital on high school completion that compared recent immigrants, long term immigrants, native born persons with at least one foreign born parent and native born persons of native parentage, White and Kaufman (1997) found that those who were bilingual had a $17 \%$ less likelihood than those who spoke only English to be high school dropouts. Thus again, as previously noted, language proficiency appears to play a significant role in school outcomes and adaptation of immigrant and refugee children.

In reviewing literature on language proficiency specifically related to refugee children, Wilkinson's (2002) study on the factors influencing the academic success of refugee youth in Canada found that the influence of limited knowledge of English on arrival to Canada has devastating consequences for the educational experiences of refugee children and youth. A study in Calgary by Watt and Roessingh (1994) found that $74 \%$ of ESL students did not complete high school, a finding that disproportionately affects refugees over other students. Derwing, DeCorby, Ichikawa, \& Jamieson, (1999) in their study of ESL high school students over a 5-year period found that only $40 \%$ received a diploma on completing high school, $46 \%$ did not complete high school at all, and $14 \%$ completed with insufficient credits to graduate from high school. Recent research suggests that lack of English language proficiency is a predominant influence on academic problems of refugees and immigrants (Huang, 2000; Watt \& Roessingh, 1994; West et al., 2000; Wilkinson, 2002). Wilkinson (2002) stated 
that children without language problems are more likely to successfully integrate and also do better at school and in subsequent employment.

In his articles on empirical and theoretical underpinnings of bilingual education, Cummins $(1981,1992)$ explains that basic interpersonal communicative skills (BICS) and cognitive/ academic language proficiency (CALP) are qualitatively different skills. He explains that BICS include skills such as pronunciation, basic vocabulary, and grammar that are required in everyday communications which most immigrant students develop rapidly resulting in the teachers prematurely assuming that minority children have attained sufficient English language proficiency to exit to an English-only program. He explains that CALP is a cognitively demanding process that is not embedded in a meaningful interpersonal context. Finally, his review of numerous research studies point to the interdependence of native and second language learning in advancing CALP skills and indicated that second language CALP takes five years or more to develop. Thus it would make sense that with increasing length of time in the United States, children may acquire increased language proficiency if given the learning opportunities.

Olsen (2000) discussed the psychosocial consequences of the push by both U.S. schools and immigrant parents for non-native children to learn English, explaining that for these children English acquisition is a symbol of belonging to their new culture. However, she also notes that often families discover that becoming fluent in English is accompanied by a loss of home language use, fluency, and development. She adds that teasing by U.S. peers causes many 
students learning English to feel shame and to become silent to avoid humiliation. Further, ESL classes are often separated from mainstream classes leading to fewer opportunities for newcomers to learn the slang used by their peers, and consequently they find they are unable to communicate with U.S. students. Accordingly, Olsen concludes that immigrant and refugee students may find themselves struggling to communicate well in their native language and in English.

Most of the literature on language acquisition does not specifically address refugees; rather, it is mostly applied to all students coming from countries in which the host country's language was not the student's first language (McBrien, 2005). These studies suggest that immigrant students with good English language skills were better adjusted to their U.S. school environments. For example, in studying alienation in Vietnamese, Laotian, Hmong, and Cambodian refugees, Nicassio (1983) found a strong correlation between alienation and insufficient English skills. The Laotian and Vietnamese participants who demonstrated better English language proficiency, tested lower on the alienation measures than Hmong and Cambodian participants. Similarly, Pryor's (2001) study of Bosnian, Albanian, and other refugee and immigrant students in a Michigan city found that the children viewed English acquisition to be important to their future success in their new country, more than half of the children said that English acquisition was difficult for them, and more than $80 \%$ said they helped their parents with English skills. Moreover, the parents reported ridicule, harassment, and embarrassment because they were not fluent in English. 
Portes and Rumbaut (2001) explained language acquisition in the context of psychosocial adjustment and identity. They discussed how children's language acquisition related not only to academic achievement but also to their success with acculturation and a sense of continuity with their parents and others from their native country. They noted that Bilingual children had the highest test scores, lowest levels of depression, highest self-esteem, and highest education and career goals. They also had the fewest conflicts with their parents. Further, they criticized the tendency of U.S. school policies toward English immersion as it increases cultural dissonance and can cause children not only to lose their native language, but also to fall short of acquiring full proficiency in English.

Lastly, McBrien (2005) summarizes that refugee children's psychosocial adjustment and language acquisition are affected by experiences of trauma and the availability of parental and social support. She notes that recent researchers consider school settings that do not require rapid language and cultural acquisition to be the best settings for refugee children. She adds that the general consensus in the literature is that using cultural elements from the students' native countries facilitates language acquisition. She reports that language is a major barrier to learning until children become competent in speaking, reading, and writing English. Finally, she states that in the case of language acquisition, segmented assimilation depends on community resources as well as cognitive ability.

Cultural Identity. In going back to the Birman and Trickett's (2001) differentiated multidimensional model of acculturation, cultural identity is the 
second distinct aspect of the acculturation process. Thus, all the previously discussed studies on acculturation employing the differentiated multidimensional model of acculturation have also looked at cultural identity and a summary of the findings and the literature in general is presented below.

In their study of the acculturation process of Jewish adolescents and their parents, Birman and Trickett (2001) found an unexpected acculturative gap that was observed between parents and children with respect to Russian identity, with adolescents being more identified with the Russian culture than did their parents. For adolescents, there appeared to be a gradual increase in identification with American culture with length of residence, but their identification with the Russian culture was not significantly related to length of residence. The authors also noted that the overall level of Russian identification was substantially higher than American indicating that the adolescents appeared to continue to identify more strongly with the Russian culture than with the American culture, even after having lived in the country for a long time. For the parents on the other hand, there was a trend suggesting an increase in American identity with length of residence and a significant decline in Russian identity with length of residence.

Birman and Trickett (2001) speculate that the importance of identity in adolescence in general or the fact that the adolescents in the study immigrated when very young and did not have a choice about immigrating to a new country may explain their tendency to maintain strong identification with the Russian culture. Moreover, given that the parents freely chose to immigrate to the United States, they may be more eager to give up their Russian identity to embrace an 
American identity. Thus, Birman and Trickett reinforce the notion that the processes of acculturation, and in this context, cultural identity, differ for adolescents and adults.

In their study with the same Jewish refugee population in looking at the potential role of community differences in the acculturation and adaptation processes, Birman et al., (2005) found that Russian identity for the adolescents did not differ by community. This was similar to the results Vinokurov et al. (2002) found in their study that looked at the psychological and acculturation correlates of work status among Soviet Jewish refugees in the United States. The authors explain that the lack of community differences in Russian identity may be attributed to cultural identity being an aspect of the acculturation process that is relatively stable over time. However, the authors also report an important community difference with respect to the adolescents' American identity. American identity was substantially higher in the dispersed community than in the concentrated community even though American identity was in general higher for Jewish participants, a smaller percentage of whom resided in the dispersed community. Overall, the authors conclude that for these adolescents, the community rather than the ethnicity played a role in their cultural identity development.

Another key finding in the above study was that higher American identity for these adolescents predicted higher grades and fewer school absences in the concentrated community, but not in the dispersed community. Birman and Trickett (2005) suggest that the students who succeeded academically in the 
concentrated community may have either succumbed to or embraced an assimilationist pressure (i.e., encouraging American acculturation while discouraging attachment to Russian culture). Further, they discuss that for the adolescents in the dispersed communities, being more American may have been less important, given the community had a wide range of cultural identities that appeared to be very visible and accepted.

In contrast, the community context was less of an influence on the rate of loss with respect to identification with and attachment to Russian culture. Further, the authors found a community difference emerge with respect to the relationship between American and Russian cultural identity, i.e., the two identities were significantly and substantially negatively related in the concentrated community, but unrelated in the dispersed community. The authors comment that the pattern in the concentrated community suggests that as American identity is acquired, it displaces Russian identity reflecting an assimilation pattern. The authors note that such a pattern may indicate cultural conflict where identifying with one culture is in conflict with identifying with the other and so some adolescents within some community contexts such as a concentrated community may experience an environmental press to choose their allegiance (Birman, Trickett, \& Buchanan, 2005). Consequently, through this study's findings, the authors bring to attention the idea of context presenting a press for the individuals to acculturate in a certain direction.

On a slightly different note, Trickett and Birman (2005) comment on the considerable attention that has been given to the question of whether children 
adapt better to school when they retain their ethnic identity. Several studies have reported a positive relationship between ethnic identification and academic success; these include Sung (1987) and Ogbu (1991) for Chinese American adolescents in New York and Oakland, CA; Gibson (1988) for Punjabi children in northern California; and Matute-Bianchi (1986) for Mexican American adolescents in California (Bankston \& Zhou, 1997; A. Portes \& Zhou, 1993). However, Portes and Zady (1996) noted no effect of maintenance of one's cultural identity on academic achievement in a sample of second-generation eighth and ninth graders from eight cultural categories (e.g., Southeast Asian, other Asian, Jamaican, Haitian). Wood and Clay (1996) found a negative relationship between American Indian identity and grades. While looking at the role of American identity in school achievement, Portes and Zady (1996) found that across several immigrant groups, a higher level of American identity had a negative effect on academic achievement. Wood and Clay (1996) on the contrary found that socialization into society (American acculturation) was positively related to grades and school achievement in their American Indian sample. Thus, it appears that the findings regarding cultural identity and school adjustment and performance are inconsistent across different samples and studies.

Persky and Birman (2005) explored ethnic identity as an aspect of acculturation among 351 Russian-speaking Jewish refugees from the former Soviet Union in the United States. In addition to the usual bidirectional model that considers the two identities, i.e., ethnic identification with the culture of origin (Russian) and identification as a member of one's new society (American), 
Persky and Birman broadened the study's examination of identity to include Jewish identity as a third component. The findings indicated that it was their Jewish identity as opposed to Russian and Israeli identities that was most salient to these adolescents' self concept and feeling comfortable in the larger U.S. society. The authors note that the findings relating stronger sense of Jewish identity to less alienation were consistent with the existing notion that selfidentification with pride in one's ethnicity carry over to feelings of belonging and contentment. Further, their findings suggested that the other two dimensions of identity, Russian and American, also played a role in feelings of alienation from the new society, where in greater sense of Russian identity was predictive of greater alienation from the new society; on the other hand, greater American identity was predictive of less alienation. Thus again, as Persky and Birman (2005) emphasized in their study, the process of acculturation encompasses not only the behavioral and linguistic domains but also the attitudinal/ identity realm.

Behavioral Acculturation. Trickett and Birman (2005) report that behavioral acculturation, or participation in activities associated with one culture or the other, have been found to affect school adaptation. They state that integration into American peer networks represents one inroad to participation in American culture for these children. The actual findings in this area have been somewhat inconsistent. For instance, Bankston and Zhou (1997) in their study examining the social adjustment of Vietnamese American adolescents found that association with either American or Americanized Vietnamese friends had a negative effect on school adjustment, which included a measure of academic 
performance. Gibson reported positive academic consequences of not socially integrating American peer group in her study of Punjabi Sikh immigrant adolescents in California. Eisikovits (1995) however reported finding that a lack of social integration with Israeli adolescents in school did not affect academic performance on a sample of Soviet Jewish adolescents in Israel. Thus, suggesting that behavioral acculturation interacts in complex ways with peer contexts to yield differing outcomes in differing contexts (Trickett \& Birman, 2005).

Birman and Trickett (2001) found that with respect to behavioral acculturation, for their Russian adolescent participants, American behavioral acculturation increases and Russian behavioral acculturation gradually declines with length of residence. The authors find that the shift from being more Russian oriented to becoming more American oriented behaviorally occurs after approximately 4-5 years in the country. The level of American behavioral acculturation was found to be higher than Russian behavioral acculturation. The results also indicate that for parents, Russian behavioral acculturation declines and American behavioral acculturation increases with length of residence. Although the findings seem to suggest that after about 7 years in the country, behavioral acculturation to both cultures becomes somewhat balanced, the level of Russian behavioral acculturation is higher than American.

In their study of the role of community differences in acculturation, Birman et al. (2005) found community differences with respect to behavioral acculturation, with the level of Russian behavioral acculturation being higher in 
the concentrated community and the level of American behavioral acculturation being higher in the dispersed community. These results replicated the prior work by Vinokurov et al., (2000) and the authors believe the results are most likely due to greater opportunities for involvement with Russian culture and Russianspeaking settings in the concentrated communities in contrast to the dispersed community that may have provided greater opportunities and perhaps even necessitated participation in American cultural behaviors (Birman et al., 2005).

In summary, the researcher has reviewed the theories of acculturation and discussed the differentiated multidimensional model of acculturation, summarizing seminal work addressing language, behavioral and identity acculturation. In conclusion, given that acculturation is a complex, multidimensional process, the literature on the topic is vast and the findings varying and the discussion so far has been focused primarily on the acculturation of refugee and immigrant youth. Because the current study seeks to explore the relationship between acculturation and school adaptation of Somali Bantu refugee children, a specific aspect of refugee and immigrant adaptation needing further elaboration is their school performance. Given that an important goal of the studies on children's acculturation and school adjustment is to predict better future outcomes, a brief summary of the literature on academic achievement in general and a more detailed summary relating to refugee and immigrant school performance and outcomes is presented in the remainder of the chapter.

\section{Refugee \& Immigrant School Outcomes}

Academic Achievement 
There is consensus that children's successful school transition during the early grades is particularly important because children's grades in the initial years of school substantially affect subsequent achievement trajectories (Alexander \& Entwistle, 1988; Alexander, Entwistle, \& Danber, 1993; Ensminger \& Slusarick, 1992; Normandeau \& Guay, 1998; Reynolds \& Bezrucko, 1993; Willms \& Jacobsen, 1990).

Researchers have established links between children's academic adjustment and achievement in the early educational classroom and a variety of factors including: IQ (Kaplan, 1993), SES (Stipek \& Ryan, 1997) and the home environment (Kellaghan, Sloane, Alvarez, \& Bloom, 1993; Martini, 1995). As Feldman and Wentzel (1990) and Tremblay, Masse, Perron, LeBlanc, Schwartzman, and Ledingham (1992) note, poor school achievement may also constitute a risk factor for mental health difficulties in later childhood or adolescence. Normandeau and Guay (1998) emphasize too the particular attention in the research literature given to studying the relationship between family processes (e.g. the affective climate, the cognitive simulation, the duality of the parent child interaction, and parental involvement) and characteristics (e.g. socioeconomic level) on school achievement (Crane, 1996; Gorges \& Elliott, 1995; Grolnick \& Ryan, 1989; Pianta \& Harbers, 1996).

Refugee Youth in Resettlement

In her study on the protective factors of refugee youth in resettlement post exposure to war, Kia-Keating (2006) finds social support as an important variable that moderates the relationship between exposure to adversities and adverse 
mental health sequel, as well as positive functioning after resettlement. KiaKeating (2006) notes several social support-related variables, including impact of parental well-being and family separations and mothers' symptoms and abilities to cope as being correlated with children's symptoms and stress levels. Further, whether refugee youth live with family members or not has also been found to be associated with psychiatric symptomology, with increased symptoms occurring when a refugee youth lives with a foster family or alone (Kia-Keatinig, 2006; Kinzie, Sack, Angell, Manson, \& Rath,1986). Tousignant, Habimana, Biron, Malo, \& Sidoli-LeBlanc, (1999) found that in their study, resettled refugee boys living with their biological parents were significantly less symptomatic (at a rate of five times lower) than boys living in any other arrangement. In their study on Mayan refugee children living in camps or peer neighborhoods in Mexico, Melville and Lykes (1992) found that these children identified parents and relatives as their primary supports during difficult times, followed by friends or caregivers. These findings all further point to the importance of the availability of parental social support for the child (Kia-Keating, 2006).

In addition to support from parents, peer relationships also appear to mitigate the relationship between war exposure and mental health (Almqvist \& Broberg, 1999; Birman, Trickett, \& Vinokurov, 2002). Kia-Keating (2006) states that for refugee and immigrant youth, who often view school as their 'second family' and the most important factor contributing to their future success, education takes on a prominent role in preserving hope (Duncan, 2000; SuarezOrozeo \& Suarez-Orozeo, 2001). 
Factors Related to Refugee/ Immigrant Children's School Outcomes

Psychological Well-being. In an initial five year follow up study examining the physical, mental and psychosocial health of 643 Vietnamese refugee children in the United States, Sokoloff, Carlin and Pham (1984) concluded that those children were doing better than the researchers had anticipated emotionally as well as with regard to their health. The authors noted that the social and school problems were minimal for most of the children, especially those who were adopted and that advanced preparation and guidance given by the involved agencies (school, peers, parents, community) appeared to be significant in achieving these results. A closer examination of the subjects explained the unanticipated results.

McBrien (2005) noted that more than $90 \%$ of the 643 children included in the study arrived in 1974 or 1975 , i.e., those that belonged to the first wave of exodus from Southeast Asia who were generally coming from the educated and financially well off classes. Additionally, $72 \%$ were adopted by American parents. Only $8 \%$ were with their own refugee parents ( $20 \%$ were foster children); and the mean age was 5 years and 5 months. Thus, the majority of the children were welcomed into US families as infants and toddlers. This explains Sokoloff and his colleagues' findings that most of the adjustment difficulties were experienced and overcome in the first year after immigration, concluding that the children and their families were thriving physically, developmentally, emotionally, and socially.

In contrast, Mosselson's (2002) study of 15 adolescent Muslim refugee girls from Bosnia actually sheds light on the complexity of the adjustment 
process. The study found that the girls worked hard to attain high grades and that they were cognizant that their high achievement status moved them from being viewed as alien to an identity that could blend into the general culture. Many of the girls stated that they worked hard to avoid the spotlight that academic deficits would create. Mosselson notes that a problem with the anonymity was that the girls' depression was overlooked. He adds that many of the girls' experiences in U.S. schools were negative and that school success did not fit with the traditional psychosocial model of poor adjustment or depression; thus, the students' needs were ignored. He concluded that the students were succeeding academically, but their psychosocial adjustment was poor.

Similarly, Bankston and Zhou (2002) analyzed data from the 1995 National Longitudinal Study of Adolescent Health for relationships between selfesteem, psychological health, and academic achievement of White, Black, Asian and Latino students. They found that Asian students had the highest academic success, but also exhibited the highest levels of depression and lowest levels of self esteem. Like Mosselson (2002), Bankston and Zhou (2002) also concluded that doing well is not the same as being well and they reported that immigrant status affected adolescents' sense of self-worth.

Parental Factors. In addition to all the literature on language, identity and behavior and the acculturation process discussed in the early part of this chapter, it is important to note that the psychological well-being of these refugee children is greatly impacted by their traumatic pasts that are often complicated by differing experiences of parents and children. For instance, Ascher (1985) quotes a study 
by Stein (1980) that indicated that even after five years, more than $80 \%$ of the participants still had serious concerns about their separation from missing family members; nearly $70 \%$ retained stressful memories of the war and their flight from their home country; and nearly $60 \%$ were still homesick and worried about their communication difficulties with friends and families still in Asia. Fernandez-Kelly and Curran (2001) note, that once resettled, adults often took lower-skilled jobs with less status than those they had previously held in their home countries. Given the Southeast Asian "fear of losing face," many were deterred from asking for help or expressing their frustration. Asher (1985) also notes the shame of rape and culturally different gender expectations added new stresses on families. Because adults were anxious about these concerns, they were often ill-equipped to provide their children with the emotional support and positive models that they needed to succeed socially and academically.

McBrien (2005) reported that parents and parental involvement in their children's education are frequently cited factors in student success. In their data from the Children of Immigrants Longitudinal Study, Portes and Rumbaut (2001) indicated that refugee and immigrant students are positively affected by parental support and interest in their children's education even though that support is not necessarily manifested in typical U.S. ways, such as parental involvement in the schools. In a study of 402 high school students, Zhou and Bankston (2000) found that Vietnamese parents tend to pressure their children to enter highly skilled professions such as medicine or law, and that Asian students achieved higher grades in school and on standardized achievement tests (Bankston \& Zhou, 
2002). They also found that Vietnamese parental involvement in ethnic communities correlated negatively with youth involvement in underprivileged or deviant subcultures (Bankston \& Zhou, 1997).

McBrien (2005) notes that there are also some parental factors that could act as obstacles to refugee children's school success. Ascher (1985) found that because refugee parents are frequently victims of trauma, they are not always able to provide emotional support. Further, when adults do not acquire the new language as rapidly as children, parents are less able to help their children with homework. Finally, because of different cultural beliefs, many parents may understand the concept of parent involvement differently from the way the U.S. parents do. All these conditions, McBrien (2005) notes increase cultural dissonance and thereby negatively influence school success of refugee children.

In summary, as McBrien (2005) reports, parental factors of misunderstanding, conflicting cultural beliefs, and language difficulties amount to dissonant acculturation as termed by Portes and Rumbaut (2001), in which parents lag behind their children's acquisition of the language and culture of their new country. Parents may feel a loss of control and may experience identity problems when their children are forced to take on adult roles for them. Family conflicts increase, leading the children to feel a sense of loss of safety and security. Students may feel like they do not belong anywhere, as they become alienated from their parents, but are not truly accepted by their peers.

Discriminatory practices on the part of teachers and peers increase the refugee students' isolation. Researchers found that discrimination often stemmed from a 
lack of accurate information and from cultural misunderstandings (McBrien, 2005).

Culture Brokers. As noted above once refugee children are resettled, they tend to acquire conversational ability in the language of their new country faster than their parents. As a result, they often must translate at school meetings, doctor appointments, and service organizations for their adult caretakers. Many refugee children write checks for mortgage and other payments. Such role reversals between children and parents create identity confusion and conflict between the generations (McBrien, 2005; Zhou, 2001; Zhou \& Bankston, 2000).

As part of a larger research project (Birman \& Trickett, 2001; Birman, Trickett, \& Vinokurov, 2002; Trickett \& Birman, 2005; Vinokurov, Trickett, \& Birman, 2002) Jones and Trickett (2005) studied the acculturation and adaptation of refugee adolescents and their families. In their study, the authors examined 'culture brokering' (i.e., 'in immigrant families culture brokering occurs when children mediate the new culture for their family') in 226 Russian immigrant adolescent-mother dyads to determine the types and amounts of culture brokering Russian adolescents performed, why they assumed this role, and how the role affected adolescent and familial functioning.

The authors approached culture brokering from a life domains perspective and assessed acculturation as a multidimensional process with respect to both culture of origin and culture of resettlement, there by being able to assess how culture brokering is related not only to the adoption of American culture but also to the retention of the culture of origin. The results indicated that most 
adolescents (89\%) reported culture brokering for their families, and that children of families that had arrived in the US more recently, with mothers who were less American-language acculturated, culture brokered more. They found that higher levels of culture brokering related to higher adolescent stress and reports of problems at home and with friends and lower feelings of school membership.

Role of School. In his review of the literature on mental health and social adjustment of refugee children, Eisenbruch (1988) found that not only personal bereavement but also cultural bereavement, i.e., 'the experience of the uprooted person or group resulting from loss of social structures, cultural values and selfidentity' (Eisenbruch, 1991, p. 674) is an important factor in a refugee child's adjustment. Based on Erikson's (1968) theory, Eisenbruch notes the significance of "uprooting" with regard to disruption of a person's concept of self. For example he stated that adolescents may have difficulty in balancing loyalty to family with the American ideal of individual progress. Eisenbruch argued that rapid acculturation can negatively affect children's ability to complete their grieving process and claim their cultural identity. He stated that effective school programs respect the native cultures of refugee children and allow them ample time to adjust and learn the language of their host country. He concluded that schools can be centers for acculturation that, with effective teachers and programs, can reduce environmental barriers and increase child's sense of competence.

School Drop-Outs. Shanahan, Mortimer and Kruger (2002) report the significance of academic achievement and skills to career success and satisfaction. Rong and Preissle (1998) report that, according to the 1990 U.S. 
Census figures, $18 \%$ of 17 -year-old and $26 \%$ of 18 -year-old immigrants were dropouts. Based on the data from the Children of Immigrants Longitudinal Study, Portes and Rumbaut (2001) found an average of $3 \%-10 \%$ school attrition rate, depending on nationality, and $14 \%-25 \%$ "inactive" rate (this rate includes students who simply stopped going to school, although they may not have officially dropped out and also includes those who may have moved to another district without officially transferring).

As previously discussed, French and Conrad (2001) and House (2001) explain that school dropout of immigrant and refugee students result from a complex mixture of factors, including self-perceptions of their academic ability, antisocial behavior and rejection by peers and lack of psychological and academic preparation before entering U.S. schools (Rong \& Preissle, 1998). Portes and Rumbaut (2001) also reported that in spite of high educational expectations across diverse immigrant groups, some students drop out because of unsafe school conditions, dissonant acculturation when children acquire the language and cultural norms of their new country faster than their parents, poverty, and hostile social environments.

Wilkinson (2002) states that students' grade placement upon arrival and their perceptions of appropriateness of their grade placement would be an important indicator of their educational success. In her study examining the education experiences of 91 refugee youth and 123 of their parents in Canada, Wilkinson (2002) found appropriate grade placement upon arrival as correlated with academic performance. Results suggested that the respondents who felt 
their grade placement was appropriate for their age and previous educational experience when they began school in Canada are more likely to be 'on-track.' Those respondents who felt that their grade placement was too high are more likely to experience problems in school, and will tend fall behind or drop out of school.

Cultural Misunderstandings: Discrimination \& Muslim Student Experience. Cultural misunderstanding can result in prejudice and discrimination, with the result that students, already struggling with an unfamiliar language and confusing cultural changes, must also work to overcome the impact of negative attitudes (Fisher, Wallace, \& Fenton, 2000; McBrien, 2005; Ogbu, 1982; Olsen, 2000; Wingfield \& Karaman, 2001). Portes and Rumbaut (2001) found that discrimination was the greatest barrier to adaptation for immigrant and refugee students. Victims of discrimination, experience lasting effects on their selfperceptions, social interactions, motivation, and achievement (Portes \& Rumbaut, 2001; Suarez-Orozco \& Suarez-Orozco, 2001). For refugees, discrimination also affects the process of acculturation (McBrien, 2005). An area of discrimination and cultural misunderstandings lies in the Muslim students' experiences in the United States.

McBrien (2005) notes that most of the research literature on Muslim students in the United States has appeared since the terrorist attacks on September 11,2001. Media reports have documented hate crimes directed at Muslim students and other Muslim Americans (Asali, 2003; McMurtie et al., 2001; Wingfield \& Karaman, 2001). Carter (1999) reports that many U.S. born citizens 
negatively stereotype Muslims as fanatics and that U.S. born school children often tease foreign-born peers about their Arab names. He states that U.S. born students often bully Muslim students for displaying outward signs of their religion, such as wearing hijab and fasting during Ramadan. This matter is particularly relevant to the current study given the Somali Bantus are predominantly a Muslim community of Islamic faith.

The School Experiences of Somali Refugee Youth in the U.S.

In a needs assessment study of Somali youth in one Maryland community (Birman, Trickett, \& Bacchus, 2001), the researchers interviewed 14 Somali middle school and high school students, 4 parents, and 20 school staff, that also included 15 ESL teachers from three high schools, two middle schools, and two elementary schools. The researchers reported that the Somali students stated they were unprepared for the taunts and stereotypes of their U.S. born peers. They stated as an example that the U.S. students would tease the girls about their head coverings, and they would ask the Somalis if they went naked in Africa or lived in trees. Some Somali students reported negative treatment from students as a result of doing well in their classes. The students reported experiences of discrimination as a result of their religious practices, for example, their clothing, daily prayer, and refusal to date. The Somali girls related an incident in which school personnel expected them to remove their scarves for school ID photos, causing them to feel disrespected.

The students spoke with the researchers about dropping out of school, and all of them knew other Somali students who had left school before receiving 
high school diplomas (Birman et. al., 2001). Some students also cited the difficulties of understanding school subjects because of earlier gaps in their education created by their refugee experience. Other students mentioned the inability to make friends in school and the unavailability of resources for help. The authors state that the teachers who were interviewed reported being afraid of saying anything that might raise traumatic feelings in the refugee students, and they expressed instances of cultural misunderstandings. Further the teachers reported being unhappy about the lack of involvement from Somali parents, and they saw that ESL students did not mingle with American students.

The needs assessment of the Somali youth and their school experiences is the only study on Somali refugee youth's school adaptation in the United States. The current study hopes to add to this scarce acculturation and school adaptation literature on Somali Bantu refugee children in the United States. 


\section{CHAPTER III \\ METHODOLOGY}

The study's methodology is organized according to the following topics:

(a) statement of purpose; (b) selection of participants; (c) instrumentation (d) data collection and (e) research design and data analysis.

\section{Statement of Purpose}

Given the ongoing influx of refugees worldwide, refugee mental health issues have been a key area of recent psychological research. Understanding the acculturation and adaptation processes of refugee children, adolescents and adults has been characterized as an important aspect of their mental health; however, little existing research describes it in the context of Somali refugees and none exists in the context of Somali Bantu refugees. The current study intends to offer a better understanding of the adjustment of Somali Bantu refugee students in one mid-western U.S. community that has recently become home to a large number of resettled Somali Bantu refugees. Local agencies and organizations like the public school systems, community mental health organizations and medical organizations that work and interact with the Somali Bantus on a daily basis continue to want more information and knowledge on the Somali Bantus. 
Given that not much is known about this community to those that are involved in serving them directly and indirectly in various capacities, a study of this nature was essential in assessing the needs of and providing recommendations for improving the adaptation of these Somali Bantu children. The purpose of this study was to examine the acculturation and school adaptation of Somali Bantu Refugee children in a mid-western U.S. city.

This study was guided by the following research questions:

1. To what extent do Somali Bantu children experience American Acculturation at the three levels of acculturation, i.e., language, identity, and behavior, when considering demographic variables like age, gender, and years in the United States?

2. To what extent do the Somali children retain their Somali culture at the three levels of acculturation (language, identity and behavior) when considering demographic variables like age, gender, and number of years in the United States?

3. To what extent does the contribution of American acculturation to school adaptation differ from contribution of retaining Somali culture to school adaptation (school grades, ACCESS scores, and SDQ scores) based on various demographic variables like age, gender, and years in the US? Selection of Participants \& Procedure Based on prior research in the area of acculturation and school adaptation (e.g. Birman \& Trickett, 2005; Birman et al., 2005) the sample for this study included 175 Somali Bantu refugee children enrolled in five elementary schools 
in a mid-western city in the United States. For the purpose of this study, the five schools will be identified as School A, B, C, D, \& E. Various officials from the city's public school system as well as their ESL department (ESL Teachers and ESL Academic Consultant) and the Director of a local Community Center that is residence to a large number of Somali Bantus, served as resources in providing recommendations and contacts for the five elementary schools with a Somali Bantu refugee enrollment.

To minimize the number of factors that could influence the validity of the study, certain criteria were employed in the selection of the participants. Given that the Somali Bantu refugees share social, cultural and historical experiences unlike other ethnic Somali refugee children, the study was limited to Somali Bantu refugee children only. Further, to avoid additional effects of age, the study restricted its sample to elementary-aged school children only.

Prior to conducting the research activities, approval was obtained from the Human Subjects Committee at the University of Louisville as well as from the Jefferson County Public School System explaining the intent, the process, and the value of a Somali Bantu refugee child's participation in the research. The research may possibly influence future training and teaching of ESL teachers and mental health professionals teaching or counseling Somali Bantu refugee children and policy development related to these children's acculturation and school adaptation.

Initial contact was made with the school principals as well as the ESL teachers of the respective schools to present the proposed study and to obtain 
their approval and support to collect data. Informed consents were sent to the parents of the Somali Bantu children in each of the five schools for parental approval for their child's participation in the study. In addition, all the children over age seven were asked to sign an assent form as is required by the Internal Review Board. Based on Birman et al.'s (2001) research on the Somali youth in Maryland, the Somalis are very community oriented people. With this in mind, ongoing contact with the community leaders to seek their approval and support for the study was an essential step in the process. The community leaders and members were consulted to build rapport and trust as well as to learn about their perspectives on the needs and adjustment of their children in the United States as was done by Birman et al. (2001). The community leaders showed eager interest in the study and were willing to assist in any ways they could. Further, the community leaders, director of the community center, as well as the ESL staff together helped in identifying a certified Somali Bantu interpreter and translator for the study.

The acculturation measure (i.e., The Language, Identity and Behavioral Acculturation Scale; LIB) was completed by the children and was administered in Mai-Mai with the assistance of a Somali Bantu interpreter fluent in English and Mai-Mai during class periods. Based on the recommendations of various school personnel at the elementary schools including the ESL and mainstream teachers, counselors, and other relevant staff who were interviewed, the interpreter orally administered the measure to a group of about 5 children at a time (Birman, Trickett, \& Bacchus, 2001). Prior to data collection and in keeping with the 
recommendations in doing cross-cultural research, the scale was translated from English to Mai-Mai, and back translated to English by two different certified individuals to ensure cross-cultural validity of the measure (Brislin, 1970, 2000; Cha, Kim, \& Erlen, 2007).

As noted by Rice and Broome (2004), providing potential research participants with incentives maximizes participation and minimizes attrition over the life of the study. Thus, as suggested by the ESL teachers and other involved ESL professionals, each participant was provided a piece of candy as an incentive to participate in the study.

The ESL teachers were requested to complete the Strengths and Difficulties Questionnaire in English on each of their participating students. Student demographics were obtained from school records. Additionally, student grade cards for data on their grades on Social Behavior and Work Study Habits were obtained for each child from the school administration. Student records were obtained from the ESL Office for each student's scores on the ACCESS for ELLs (Assessing Comprehension and Communication in English State to State for English Language Learners). All names, student and teacher responses and other obtained information were kept strictly confidential and the data were stored in a locked filing cabinet in Dr. Thomas Reio's office.

The current study followed a mixed methods design. As Greene, Caracelli, \& Graham, (1989) note, mixed methods designs allow for triangulation, the opportunity to examine overlapping and different facets of a phenomenon, and discovering different paradoxes, contradictions or fresh perspectives. Brewer and 
Hunter (1989) note that there is a need for a more integrated methodological approach to research emphasizing that individual researchers combine methods in their investigations rather than using mono-method investigations. Empirical evidence documents that using both quantitative and qualitative research methodologies can lead to convergent evidence concerning the importance of the psychological construct being measured (Tashakkori \& Teddlie, 1998). In their discussion of the threats to validity of research results, Cook and Campbell (1979) point out that one of the shortcoming to using mono-methods study is that it poses a threat to construct validity of "putative causes and effects" (p. 66) i.e. that measuring a construct using only one method makes it difficult to differentiate the construct from its particular monomethod operational definition. Thus, Campbell and Fiske (1959) emphasized the importance of having multiple quantitative methods for assessing psychological traits. Lastly, Denzin (1978) applied the term "triangulation" to the process that involves combining data sources to study the same social phenomenon.

Thus as part of a mixed methods design, the current study employed semi-formal interviews with open ended questions as part of data collection to enhance the understanding of the quantitative findings. The individuals that were interviewed included the various ESL teachers, a few class teachers, school principals, ESL Academic Advisor for the local public school system in the district, various individuals in the community including Somali Bantu leaders, mental health school counselors, director of a community center who at the time was intimately involved with the Bantu population as well as parents/ family 
members of the students. Important qualitative findings are reported in chapter 4 and discussed in chapter 5 .

Instrumentation/ Measures

Demographics

Demographic information was obtained from each child's school records, including age, gender, length of time in the U.S., and which of the five schools the child was enrolled in.

Acculturation

Acculturation was measured using a modified version of The Language, Identity, and Behavioral acculturation Scale (LIB; Birman \& Trickett, 2001). The LIB has been previously used in several studies with a Soviet Russian Jewish adolescent refugee population to assess their acculturation to the Russian and American cultures independently (Birman et al., 2002; Vinokurov et al., 2002). The scale consists of fifty items measuring acculturation to Russian and American cultures independently, resulting in an overall American Acculturation Index (AAI) and an overall Russian Acculturation Index (RAI). In the Trickett \& Birman (2005) study, the reliabilities reported for the overall AAI and RAI were .90 and .94 respectively. For the current study, the Cronbach's alpha reliability coefficients were .89 for the entire scale, .86 for the overall American Acculturation Index (AAI) and .86 for the overall Somali Acculturation Index (SAI).

The Language Acculturation subscales consist of nine parallel items asking respondents to rate their ability to speak and understand Russian and 
English. Ratings are made on a 4-point Likert scale ranging from 1 (not at all) to 4 (very much). Birman and Tricket's (2005) study reported Cronbach's alpha reliability coefficients of .90 for the English and .95 for the Russian language subscale. Cronbach's alpha reliability coefficients for the Somali Bantu children in this sample were .78 for the English and .71 for the Somali Bantu (Mai Mai) Language subscale.

The Identity Acculturation subscales consist of seven parallel statements regarding the degree of identification with each culture (e.g., "I consider myself American/ I consider myself Russian") and the extent to which respondents regard this identification positively (e.g., "I am proud to be American/I am proud to be Russian"). Ratings are made on a 4-point Likert scale ranging from 1 (not at all) to 4 (very much). Trickett $\&$ Birman (2005) report alpha reliability coefficients of .92 for Americans and .93 for Russian identity. Consistent with Trickett and Birman's findings, (2001) Cronbach's alpha reliability coefficients for the Somali Bantu children in this sample were .97 for American cultural identity and .89 for the Somali Bantu cultural identity subscale.

Behavioral acculturation asks participants to rate the extent to which they engage in behaviors associated with each culture (e.g., language use, media, music, entertainment, food). The scale consists of nine items regarding American culture and nine parallel items concerning Russian culture. Again, the ratings are made on a 4-point Likert scale ranging from 1 (not at all) to 4 (very much). In the Trickett \& Birman (2005) study, the reliabilities reported were .76 for the American subscale and .86 for the Russian subscale. Cronbach's alpha reliability 
coefficients for the Somali Bantu children in this sample were .70 for the American subscale and .75 for the Somali Bantu subscale.

The instrument was developed with particular consideration for Russian immigrants. Currently, no established norms for the scores on the LIB are available (Miller, Sorokin, Wang, Feetham, Choi, \& Wilbur, 2006).

In the proposed study, a Somali Bantu translator translated the items into Mai-Mai, replacing Russian with Somali Bantu. For example, instead of the original statement asking the participant to rate their ability to speak Russian "overall," "with family," "with Russian friends," "on the phone," and "with strangers," the participants were asked to rate their ability to speak Mai-Mai "overall," "with family," "with Somali Bantu friends," "on the phone," and "with strangers." Thus, the scales resulted in an overall American Acculturation Index (AAI) and an overall Somali Bantu Acculturation Index (SAI) score, respectively. Given the difficulty in having elementary school aged children attend to a task for a long time, the shortened 8 item version of the identity sub-scale was used. Further, based on Cha et al.'s (2007) discussion and recommendations on translation of scales in cross-cultural research and the involved issues and techniques and for the ease in understanding and responding to the items, a 3point Likert scale was used ranging from 1 (a lot), 2 (sort of) and 3 (no), rather than the 4-point Likert scale used in the original instrument. 
School adaptation was assessed by looking at the Total Difficulties score on the SDQ, School Grades for Social Behavior and Work Study Habits, and the Proficiency Level Composite scores for 2006-07 on the ACCESS for ELLs.

Strengths and Difficulties Questionnaire. Each of the five ESL teachers at the participating Elementary Schools were requested to complete the Strengths and Difficulties Questionnaire (SDQ) on each of their Somali Bantu students. The SDQ is a brief behavioral screening measure that asks about twenty five attributes, some positive and others negative. The twenty five items are divided between five scales of five items each, generalizing scores for conduct problems, hyperactivity, emotional symptoms, peer problems, and pro-social behavior; all but the last are summed to generate a total difficulties score (Goodman, 1997, 2001). Three versions of the instrument exist: the self-report for ages 11-17, the parent or teacher form for ages 4-10, and the parent or teacher form for ages 1117. For the purpose of this study, the Teacher and Parent Form for ages $4-10$ was employed.

The instrument was normed on a sample population of 403 parents of children attending one of two London child psychiatric clinics or the children's department of a London-based mental hospital on a Caucasian population. The range of test-retest value was reported as being $.70-.85$; and the range of internal consistency reported is $.51-.76$. Further, criterion validity was assessed and found to be acceptable (Goodman, 1997; 2001). Internal consistency for each of the five subscales has been shown to be good with a mean Cronbach's alpha of 0.73 (Goodman, 2001). In the current study, the mean Cronbach's alpha 
was .82. Further the Cronbach's alpha reliability coefficient for the Somali Bantu children in this sample was .90 for the Total Difficulties scale.

Work Habits and Social Behavior. A second and third school outcome for this study included school grades on Work Habits and Social Behavior. Given that language tasks are more difficult when they are context reduced and cognitively demanding, academic language tends to be more challenging in general for English as Second Language (ESL) students (Garnett \& Ungerleider, 2008). Further, because research documents that it takes learners about five to seven years to achieve grade level equivalency in academic language with native speakers (Collier, 1987; Cummins, 2000; Garnett \& Ungerleider, 2008; Klesmer, 1994), academic grades are not truly representative of ESL students' academic performance. Accordingly, the academic grades were not considered for the study, rather the grades for conduct, work and study habits were used as additional indicators of school adjustment.

The Social Behaviors and Work Study Habits grades were obtained from the student's school report card for the academic year 2006-07. The student's class teacher, and not their ESL teacher, reported their grades on the two subjects, allowing for independence of raters. The grades ranged from 1 through 6, with 1 for Outstanding progress and an observed strength; 2 for Above Average progress and exceeding expected skill level; 3 for Satisfactory progress, meeting expected skill level and usually an observed strength; 4 for Below Average/ Little Progress; 5 for Needs Improvement, making progress toward meeting the expected skill level, but needs extra time and practice in the learning 
area; and 6, Unsatisfactory, performance not meeting expected skill level, progress below age-appropriate.

English Language Proficiency. Given that the literature reports English language proficiency as a key predictor of academic success (Garnett \& Ungerleider, 2008; Rumberger \& Larson, 1998) in ESL students, the students' Proficiency Level Composite scores for 2006-07 on the ACCESS for ELLs was included as the fourth measure of school adaptation. ACCESS for ELLS stands for Assessing Comprehension and Communication in English State-to-State for English Language Learners and is a large-scale English language proficiency test anchored in the WIDA English Language Proficiency Standards for English Language Learners in Kindergarten through Grade 12 (Gottlieb, Cranley, \& Oliver, 2007). An individual student's results on the ACCESS for ELLS are reported in three ways: Raw Scores, Scale scores and English Language Proficiency Levels. Proficiency level scores are interpretive scores and thus are what were used for this study. Although proficiency levels are reported for the four language domains (Listening, Speaking, Reading and Writing), as well as four combinations of language domains (Oral Language, Literacy, Comprehension, and Overall or Composite Score), only the overall Proficiency Level Composite scores were used for the current study due to a small sample size.

The Proficiency Level Composite scores ranged between 1 though 6 , with scores over 1 and below 2 falling under the "Entering level"; scores over 2 and under 3 falling under the Beginning level; scores over 3 and under 4 falling under 
the Developing Level; scores over 4 and under 5 falling under the Expanding Level; scores over 5 and under 6 falling under the Bridging level; and a score of 6 indicating the student is at the Reaching Level, and is an indicator that the student is no longer needing ESL services due to meeting English language proficiency.

\section{Data Collection}

Parents completed the informed consent forms approving their child's participation in the study. The consent forms were made available to the parents with the aid of the ESL teachers. Teachers were provided candy to give to the children as incentive for remembering to bring back signed consent forms. Many parents were also contacted in person at their homes and at the community center to discuss the study and seek approval for their child's participation. Given the language barrier, a certified interpreter was hired to facilitate this discussion as well as answer any questions or concerns the parents had.

With the assistance of the interpreter who was fluent in English, Somali, and Mai-Mai, the LIB was verbally administered in groups of about 5 children at a time. The administrator explained to the children how to respond to the instrument, and was assisted by the interpreter and the ESL teachers in responding to the items.

The ESL teachers were requested to complete the Strengths and Difficulties Questionnaire on each of their Somali Bantu student participating in the study. Prior to completing the measure on each student, the teachers also 
completed a consent form to acknowledge their willingness to participate in the study.

Student Records were used to access information on the student's grades and scores on the ACCESS for ELLs for the academic year 2006-07.

Similar to previous exploratory research in the social sciences (e.g., Reio \& Lasky, 2007), qualitative data was obtained through semi-structured interviews with various stakeholders to further enrich the researcher's understanding of the quantitative findings. These included community leaders, the Director of the community center, various school administrators including principals, school counselors, mental health counselors, ESL teachers, as well as others from the ESL office.

Challenges in Data Collection

Given this is a unique, under-studied population, data collection had its own set of challenges. First, due to language diffiqulties the researcher had to seek the assistance of a local Somali Bantu interpreter to help get parental consent for their children to participate in the study, most parents agreed to their children's participation. Still, some parents were very hesitant to sign the consent forms as they had been trained, prior to their entry into the United States, that they should be very careful about signing documents $n$ the United States. This posed a challenge and some wanted to speak with the child's ESL teacher before signing. The families have much faith in the ESL teachers as well as their community leaders. Further, many of these families live in poor neighborhoods, mostly in subsidized housing. Thus, the researcher spent dozens of hours in the 
local housing areas to gather parental consent. The poor residential neighborhoods the children live in allow for further understanding of the context of their day to day lives that include gang activities, drug and alcohol related activities, fighting, and limited resources in the communities in which they live.

Lastly, of the five schools where data were collected, schools C, D, and $E$ are located in the south end of the town generally known as the more poor neighborhoods in town; School A is a magnet school and Schools B, C, D and E are public schools.

\section{Research Design and Data Analysis}

The research design and data analysis for the present study was modeled on Birman and Trickett's (2001) study on Soviet Jewish Russian refugee adolescents. The study utilized a basic correlational design (Gall, Borg, \& Gall, 1996). The principal advantage of the correlational method is that it permits the analysis of relationships among a large number of variables in a single study. The correlational method also provides information concerning the strengths and the degree of the relationship between the variables being studied (Pagano, 1998). Correlations were run on the various demographic variables and student scores on the four school outcome variables (1. Total difficulties score 2; Grade on Social Behavior 3; Grade on Work Study Habits; and 4-Proficiency Level Composite scores to determine relationships among variables.

Next, a series of multivariate analyses of variances were performed in order to examine group differences in acculturation and school outcomes with respect to the demographic variables. Finally, a series of hierarchical regression 
analyses were run first to determine the unique variances and effects explained by the four demographic variables (age, time since first entry into the United States, gender and school) and secondly, to predict overall American and Somali acculturation as well as acculturation at the more differentiated levels of language, identity and behavior (Cohen, 1988).

The results from the quantitative analyses were supplemented with a discussion of the key findings from the qualitative data. 


\section{CHAPTER IV}

\section{RESULTS}

The purpose of this study was to assess the relationship between acculturation and various indicators of school adaptation among a group of Somali Bantu elementary school refugee children. In this chapter I present analyses of data relevant to Somali Bantu children's acculturation with respect to both American and Somali Bantu cultures, and adopted a multidimensional framework of acculturation that included language competence, behavioral acculturation and cultural identity (Birman, 1994; Birman \& Trickett, 2001; Birman \& Tyler, 1994; and Trickett \& Birman, 2005).

To determine the relationships among variables, I will present correlations among the various demographic variables and students' scores on the four school outcome variables (1. Grades on Social Behavior; 2. Grades on Work Habits; 3. Total Difficulties Score on the Strength and Difficulties Questionnaire; and 4. Proficiency Level Composite scores for 2006-07 on the ACCESS for ELLs - Assessing Comprehension and Communication in English State to State for English Language Learners). In addition, I present correlations among the relationships among students overall American Acculturation Index and Somali Bantu Acculturation Index scores and the four school outcome variables. 
Next, the results form a series of multivariate analyses of variances are presented to report any significant group differences in acculturation and school outcomes with respect to the demographic variables.

A series of hierarchical regression analyses are presented to determine the unique variance explained by the four demographic variables, i.e., age, time since first entry into the United States, gender, and school in predicting overall American and Somali acculturation as well as acculturation at the more differentiated levels, i.e., language, identity and behavior.

To assess the extent to which selected variables predicted school outcomes, four hierarchical regressions were run to predict grades on social behavior and work study habits, total difficulties score on the Strengths and Difficulties questionnaire, and proficiency level composite scores for 20062007 on the ACCESS for ELLs. Demographic variables of age, years in the United States, and gender were entered in Block 1, schools (dummy coded) were entered in Block 2 and acculturation was entered in Block 3.

The study was designed to answer the following three research questions:

1. To what extent do Somali Bantu children experience American Acculturation at the three levels of acculturation, i.e., language, identity, and behavior, when considering demographic variables like age, gender, and years in the United States?

2. To what extent do the Somali children retain their Somali culture at the three levels of acculturation (language, identity and behavior) when 
considering demographic variables like age, gender, and number of years in the United States?

3. To what extent does the contribution of American acculturation to school adaptation differ from contribution of retaining Somali culture to school adaptation (school grades, ACCESS scores, and SDQ scores) based on various demographic variables like age, gender, and years in the US? In this chapter, the demographics of the participants are provided along with appropriate data presentation related to each of the research questions. A discussion of the results will be presented in Chapter 5 .

The Statistical Package for the Social Sciences $\left(15^{\text {th }}\right.$ edition) was used for all the analyses. The researcher's field notes and teachers' comments were explored to help inform and support the findings of the quantitative data. Demographic Variables

Demographic information is provided for a description of the participants in terms of their age, time since first entry into the United States, gender, and school of attendance. Data were obtained on a total of 175 Somali Bantu children from 5 elementary schools (School A - E) in a Midwestern city.

Of the 175 students, 21 attended School A (12.0\%), 25 attended School B (14.3\%), 44 School C (25.1\%), 37 School D (21.1\%), and 48 School E (27.4\%). The sample's distribution in terms of gender was - males $50.9 \%(n=89)$ and females $48 \%(n=84)$ of the sample, with gender information missing on two subjects. 
With regard to the grade level distribution of the sample, $32(18.3 \%)$ of the students were in kindergarten, $33(18.9 \%)$ were in first grade, $32(18.3 \%)$ were in second grade, 35 (20.0\%) were in third grade, 21 (12.0\%) were in fourth grade, and $19(10.9 \%)$ were in $5^{\text {th }}$ grade. Grade level information was missing on 3 students.

On examining the distribution of the Proficiency Level Composite scores on the ACCESS for ELLs (Assessing Comprehension and Communication in English State to State for English Language Learners), 32 students (18.3\%) fell in the Entering Level, 40 (22.9\%) fell in the Beginning Level, 33 students (18.9\%) fell in the "Developing" Level, 35 students (20.0\%) fell in the "Expanding" Level, and 6 students (3.4\%) fell in the "Bridging" Level.

The mean age in months of the sample $(N=174)$ was 9 years 2 months $(S D=2$ years 1 month). The sample age ranged between 5 years 7 months and 14 years 5 months. The average length of time the students have been in the United States was about 2 years 8 months for the sample ( $S D=9$ months), with 6 months being the shortest length of stay and 7 years 2 months being the longest length of stay. Further, for the purpose of data analyses, age and length of time in the United States were also broken down into three and two approximately equal age ranges, respectively. The age categories included the following:

1. 5 years 1 month to 8 years 0 months;

2. 8 years 1 month to 10 years 6 months; and

3. 10 years 7 months to 14 years 5 months. 
The length of time in the United States categories included -1.6 months to 3 years 9 months; and 2. 4 years 0 months to 7 years 2 months. A summary of the demographic information is presented in Table 1.

Table 1

Demographic Information

\begin{tabular}{|c|c|c|c|c|}
\hline Demographic Categories & $\begin{array}{l}\text { Frequency } \\
\text { Missing }(N)\end{array}$ & $\%$ of Total & $N(175)$ & \\
\hline Age & & & 174 & 1 \\
\hline $67-96$ Months & 59 & 33.7 & & \\
\hline 97 - 126 Months & 71 & 40.6 & & \\
\hline $127-173$ Months & 44 & 25.1 & & \\
\hline Gender & & & 173 & 2 \\
\hline Male & 89 & 50.9 & & \\
\hline Female & 84 & 48.0 & & \\
\hline School & & & 175 & 0 \\
\hline School A & 21 & 12.0 & & \\
\hline School B & 25 & 14.3 & & \\
\hline School C & 44 & 25.1 & & \\
\hline School D & 37 & 21.1 & & \\
\hline School E & 48 & 27.4 & & \\
\hline Grade & & & 172 & 3 \\
\hline Kindergarten & 32 & 18.3 & & \\
\hline First Grade & 33 & 18.9 & & \\
\hline Second Grade & 32 & 18.3 & & \\
\hline Third Grade & 35 & 20.0 & & \\
\hline Fourth Grade & 21 & 12.0 & & \\
\hline Fifth Grade & 19 & 10.9 & & \\
\hline Time in the United States & & & 170 & 5 \\
\hline $6-43$ Months & 87 & 49.7 & & \\
\hline $44-86$ Months & 83 & 47.4 & & \\
\hline Proficiency Level Composite & & & 146 & 29 \\
\hline Entering & 32 & 18.3 & & \\
\hline Beginning & 40 & 22.9 & & \\
\hline Developing & 33 & 19.9 & & \\
\hline
\end{tabular}


Expanding Bridging

Table 2 presents the means and standard deviations for variables used in the study. Similar to the Russian study by Birman and Trickett (2001), paired $t$ tests were run to compare students' acculturation scores for Somali and American acculturation. Paired $t$-tests revealed that the students' overall level of Somali acculturation (SAI), Somali Language competence (SLA) and Somali identity (SIA) were significantly higher than their American counterparts, $t=9.87$, $p<.001 ; t=7.65, p<.001 ; t=10.34, p<.001$, respectively. No differences in American and Somali behavioral acculturation (ABA and SBA) were observed, suggesting that as a group the students were relatively balanced with respect to their level of American and Somali behavior acculturation. The significant difference in the overall level of American and Somali acculturation (AAI and SAI) suggests that as a group, the students were not similar with respect to their levels of American and Somali acculturation.

Table 2

Means and Standard Deviations for Acculturation and School Outcome Variables

\begin{tabular}{llll}
\hline Study Variables & Mean $(M)$ & SD & $\mathrm{N}$ \\
\hline American Acculturation & & & \\
$\quad$ Language & 1.42 & 0.37 & 102 \\
$\quad$ Identity & 2.12 & 0.86 & 102 \\
$\quad$ Behavior & 1.52 & 0.31 & 97 \\
$\quad$ American Acculturation Index & 1.67 & 0.39 & 96 \\
Somali Acculturation & & & \\
$\quad$ Language & 1.16 & 0.24 & 102 \\
$\quad$ Identity & 1.15 & 0.38 & 102 \\
$\quad$ Behavior & 1.46 & 0.34 & 97
\end{tabular}


Somali Acculturation Index School Outcomes

Grades - Social Behavior

Grades - Work Study Habits

SDQ - Total Difficulties

Proficiency Level Composite 06-07
1.25

3.26

3.55

8.68
0.26

1.18

1.22

6.93

0.75
96

97

92

168

143

Intercorrelations among Demographic Variables, Acculturation and School

Outcomes

Similar to the Birman and Trickett's study (2001) study exploring the relationship between acculturation and school adaptation among a group of immigrant adolescents from the former Soviet Union that presented correlations of demographic variables with the outcome variables, the correlations of demographic variables with the acculturation and outcome variables in this study are presented in Table 3. Age, time since first entry into the United States and. gender are related to students' reports of acculturation and school outcomes.

Table 3 also shows a moderate (.45) correlation (Cohen, 1988, 1992) between overall American and overall Somali acculturation (AAI and SAI), suggesting these students tend to be bicultural. AAI was found to be negatively correlated with age, suggesting that older students appeared to have higher levels of overall American acculturation. AAI was also found to be negatively correlated with SDQ Total Difficulties scores, suggesting that students with higher levels of overall American acculturation were rated by their ESL teachers as having greater behavior problems/ difficulties. SAI was positively correlated with time since first entry into the United States, indicating that the longer the students had been in the US, the less likely they were of retaining their Somali 
culture. Also, a large positive correlation was found between students' level of SAI and their grades on Social Behavior (.62), indicating that students with higher levels of overall Somali acculturation were described by their ESL teachers as having better grades on Social Behavior.

Female participants were found to have better grades on social behavior and male participants were found to have more behavior problems/ difficulties based on their scores on the SDQ Total difficulties score. A large correlation (Cohen, 1988, 1992) between social behavior and work study habits grades $(.70)$ suggests that better social behavior grades are related to better work study habits grades for these students. Table 3 also shows moderate correlations (Cohen, 1988, 1992) between total difficulties score and grades on work study habits (.46) and social behavior (.49), suggesting that students with more behavioral problems/ difficulties had poor work study habits and more social behavior problems. Students' proficiency level composite scores differed based on the school they attended. The table also shows moderate correlations (.38 and .34) correlations between proficiency level composite scores and age and time since first entry into the United States (Cohen, 1988, 1992), suggesting that older students and students with increasing time in the United States, tended to have higher proficiency level composites scores for 2006-2007.

Table 3

Intercorrelations of Demographic, Acculturation, and School Outcomes Variables 


\begin{tabular}{|c|c|c|c|c|c|c|c|c|c|}
\hline Variable & 1 & 2 & 3 & 4 & 5 & 6 & 7 & 8 & 9 \\
\hline 1. Age & - & & & & & & & & \\
\hline 2. T-US & .02 & - & & & & & & & \\
\hline 3. Gender & $-.16^{*}$ & -.03 & - & & & & & & \\
\hline 4. $\mathrm{AAl}$ & $-.27^{\star \star}$ & -.04 & .04 & - & & & & & \\
\hline 5. SAI & -.11 & $.24^{\star}$ & -.05 & $.45^{\star \star}$ & - & & & & \\
\hline 6. SDQ-TD & .08 & .08 & $-.27^{* *}$ & $-.20^{*}$ & -.04 & - & & & \\
\hline 7. G-SB & -.05 & .02 & $-.22^{*}$ & .05 & $.62^{\star \star}$ & $.49^{\star \star}$ & - & & \\
\hline 8. G-WSH & -.07 & -.15 & -.11 & .12 & -.05 & $.46^{\star \star}$ & $.70^{\star \star}$ & - & \\
\hline 9. PLC 06-07 & $.38^{\star *}$ & $.34^{\star \star}$ & .09 & -.18 & .09 & -.10 & -.12 & -.16 & - \\
\hline $\mathrm{N}$ & 174 & 170 & 173 & 103 & 103 & 168 & 97 & 92 & 143 \\
\hline
\end{tabular}

Note. T-US $=$ Time since first entry in to the United States; AAI = American Acculturation Index; SAI = Somali acculturation Index; SDQ-TD = Total Difficulties score on the Strengths and Difficulties Questionnaire; G-SB = Grade on Social Behavior; G-WSH = Grade on Work Study Habits: PLC 06-07 = Proficiency Level Composite score for 06-07; $N=$ sample size; ${ }^{*} p<.05$, ${ }^{* *} p<$ .01

Comparison of Sample Demographics:

Across the 3 components of American Acculturation: A four-way betweengroups multivariate analysis of variance was performed to investigate school, age, time since first entry into the United States, and gender differences in American acculturation. Three dependent variables were used: American Language Acculturation, American Identity Acculturation, and American Behavioral Acculturation. The independent variables were school, age, time since first entry into the United States, and gender. For the analyses, the sample was broken down into two and three approximately equal categories for age and 
time in the United States respectively, to create categorical variables. Preliminary assumption testing was conducted to check for normality, linearity, univariate and multivariate outliers, homogeneity of variance-covariance matrices, and multicollinearity, with no serious violations noted. Box's test of Equality of Covariances was not significant $(p=.16)$. The multivariate tests indicated that there was a statistically significant difference between the different schools on the combined dependent variables: $F(12,156)=2.44, p=.006$; Wilk's $\Lambda=.64 ; \eta^{2} p$ $=.14$. When the results from the univariate tests for the dependent variables were considered separately, the only difference to reach statistical significance, using a Bonferroni adjusted alpha level of .017 , was American Language Acculturation: $F(4,61)=4.60, p=.003, \eta^{2} p=.23$. An inspection of the mean scores indicated that School D students had significantly lower ALA scores $(M=1.27, S D=.19)$, i.e., high American language competency compared to School E students $(M=1.61, S D=.06)$. This implied that School $D$ students had higher American language acculturation compared to School E students.

Across the 3 components of Somali Acculturation: A four-way betweengroups multivariate analysis of variance was performed to investigate school, age, time since first entry into the United States, and gender differences in Somali acculturation. Three dependent variables were used: Somali Language Acculturation, Somali Identity Acculturation, and Somali Behavioral Acculturation. The independent variables were school, age categories, time since first entry into the United States categories, and gender. Preliminary assumption testing was conducted to check for normality, linearity, univariate and multivariate outliers, 
homogeneity of variance-covariance matrices, and multicollinearity, with no serious violations noted. Box's test of Equality of Covariances was not significant $(p=.11)$.Multivariate tests revealed that there was a statistically significant difference between the different schools on the combined dependent variables: $F(12,162)=2.61, p=.003$; Wilk's $\Lambda=.62 ; \eta^{2} p=.15$. When the results from the univariate tests for the dependent variables were considered separately, the only difference to reach statistical significance, using a Bonferroni adjusted alpha level of .017 , was Somali Behavior Acculturation: $F(4,63)=8.19 . p=.000, \eta^{2} p=.34$. An inspection of the mean scores indicated that School E students had significantly higher SBA scores $(M=1.67, S D=.05)$, i.e., low retention of Somali culture behaviors compared to School $C(M=1.24, S D=.08)$ and School $D$ $(M=1.30, S D=.05)$ students. In other words, School E students had lower Somali Behavioral Acculturation levels compared to School C and D students.

Across the various School Outcomes: A four-way between-groups multivariate analysis of variance was performed to investigate school, age, time since first entry into the United States, and gender differences in school outcomes. Four dependent variables were used: Total Difficulties, Social Behavior Grade, Work Study Habits Grade, and Proficiency Level Composite for 2006-07. The independent variables were school, age categories, time since first entry into the United States categories, and gender. Preliminary assumption testing was conducted to check for normality, linearity, univariate and multivariate outliers, homogeneity of variance-covariance matrices, and multicollinearity, with no serious violations noted. Box's test of Equality of Covariances was not 
significant $(p=.26)$. Examination of the multivariate tests revealed that there were no statistically significant differences between the different schools $(F(16,126)=$ $1.51, p=.11)$, age groups $(F(8,82)=1.22, p=.30)$, time since first entry into the United States categories $(F(4,41)=.72, p=.59)$, and gender $F(4,41)=1.63, p=$ .18 ) on the combined dependent variables.

\section{Contributors to Acculturation}

Research Q1 - To what extent do Somali Bantu children experience American acculturation at the three components of acculturation, i.e., language, identity, and behavior, when considering demographic variables like school, age, time since first entry into the United States and gender?

To assess which variables predicted American acculturation, guided by theory and review of literature, a hierarchical regression was run to predict the three components of American acculturation - language, identity and behavior as well as overall American Acculturation Index. Demographic variables of age, time since first entry into the United States and gender were entered in Block 1, and schools (dummy coded, i.e., SchoolDummy1, SchoolDummy2, SchoolDummy3 and SchoolDummy4) were entered in Block 2. Results are found in Table 4.

American Acculturation Index: In the first step age, time since first entry into the United States, and gender did not account for a significant amount of variance in American Acculturation Index, i.e., overall American acculturation $\left(R^{2}\right.$ $=.00, F(3,91)=.07, p=.98)$. In the second step, school was found to explain moderately significant (Cohen, 1988, 1992) unique variance in the American Acculturation Index scores after controlling for age, time in the US, and gender 
$\left(R^{2}=.18, R^{2} \Delta=.18, F(4,87)=4.79, p=.002\right)$. Further examination of the coefficients revealed that although school variable as a whole was found to explain significant variance, individually, none of the schools were found to significantly contribute to the students' overall AAI scores.

American Language Acculturation: In the first step age, time since first entry into the United States, and gender accounted for a small (Cohen, 1988, 1992) significant amount of unique variance in American Language Acculturation $\left(R^{2}=.08, F(3,97)=2.90, p=.04\right)$. In the second step, school was found to explain moderately significant (Cohen, 1988, 1992) additional variance in the American Language Acculturation scores after controlling for age, time in the US, and gender $\left(R^{2}=.25, R^{2} \Delta=.17, F(4,93)=5.10, p=.001\right)$. Age in months was found to predict ALA, i.e., children older in age are predicted to have high American language acculturation. Further, students from School E had significantly higher scores on ALA than the students from the other schools implying School E students have lower American language competency compared to students from the other schools.

American Identity Acculturation: In the first step age, time since first entry into the United States, and gender did not account for a significant amount of unique variance in American Identity Acculturation $\left(R^{2}=.02, F(3,97)=.56, p=\right.$ .64). In the second step, school was found to explain moderately (Cohen, 1988, 1992) significant unique variance in the American Identity Acculturation scores after controlling for age, time in the US, and gender $\left(R^{2}=.17, R^{2} \Delta=.15, F(4,93)\right.$ $=4.18, p=.004)$. In regard to the school variable, students from School B had 
significantly higher scores on AIA than the students from the other schools implying these students have lower levels of American identity acculturation.

American Behavior Acculturation: In the first step age, time since first entry into the United States, and gender did not account for any significant amount of variance in American Behavior Acculturation $\left(R^{2}=.01, F(3,92)=.39\right.$, $p=.76)$. In the second step, school was found to explain moderately significant (Cohen, 1988, 1992) unique variance in the American Behavior Acculturation scores after controlling for age, time in the US, and gender $\left(R^{2}=.13, R^{2} \Delta=.11\right.$, $F(4,88)=2.86, p=.03)$. Further examination of the coefficients revealed that although school variable as a whole was found to explain significant variance, individually, none of the schools were found to significantly contribute to the students' overall ABA scores, that there may have been just about enough overlap to cause an overall significance in variance.

Table 4

Hierarchical Regression Analyses of Demographic Variables on Composite American Acculturation (AAI)

\begin{tabular}{|c|c|c|c|c|c|c|c|c|}
\hline \multirow[b]{2}{*}{ Independent Variables } & \multicolumn{2}{|c|}{ AAI } & \multicolumn{2}{|c|}{ ALA } & \multicolumn{2}{|c|}{ AIA } & \multicolumn{2}{|c|}{ ABA } \\
\hline & $B$ & $\beta$ & $B$ & 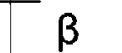 & $B$ & $\beta$ & $\bar{B}$ & $\overline{-} \beta$ \\
\hline \multicolumn{9}{|l|}{ BLOCK 1} \\
\hline Age in Months & .00 & .03 & -.00 & $-.28^{\star \star}$ & .00 & .16 & -.00 & -.10 \\
\hline Time Since First Entry into US & .00 & .11 & -.00 & .00 & .01 & .11 & .00 & .00 \\
\hline Gender & .07 & .09 & -.03 & -.04 & .22 & .13 & \multirow{2}{*}{\multicolumn{2}{|c|}{01}} \\
\hline & \multicolumn{2}{|c|}{.00} & \multicolumn{2}{|c|}{$.08^{*}$} & \multicolumn{2}{|c|}{.02} & & \\
\hline \multicolumn{9}{|l|}{ BLOCK 2} \\
\hline SchoolDummy1 & .15 & .14 & .03 & .03 & .76 & $.31^{\star}$ & -.19 & -.21 \\
\hline SchoolDummy2 & .00 & .00 & .03 & .04 & .12 & .06 & -.13 & -.18 \\
\hline SchoolDummy3 & -.24 & -.25 & -.12 & -.13 & -.36 & -.17 & -.16 & -.21 \\
\hline SchoolDummy 4 & .23 & .26 & .30 & $.36^{\star}$ & .31 & .16 & .08 & .11 \\
\hline
\end{tabular}




\begin{tabular}{|c|c|c|c|c|}
\hline $\begin{array}{l}\Delta R^{2} \\
R^{2}\end{array}$ & $\begin{array}{l}.18^{* *} \\
18^{* *}\end{array}$ & $\begin{array}{l}.17^{\star \star} \\
25^{\star \star}\end{array}$ & $\begin{array}{l}.15^{\star \star \star} \\
17^{\star \star}\end{array}$ & $.11^{*}$ \\
\hline
\end{tabular}

Research Q2 - To what extent do Somali Bantu children retain their Somali culture for the three components of acculturation, i.e., language, identity, and behavior, when considering demographic variables like school, age, time since first entry into the United States and gender?

To assess what variables predicted retention of Somali culture, a hierarchical regression was run to predict the three levels of Somali acculturation - language, identity and behavior as well as overall Somali Acculturation Index. Demographic variables of age, time since first entry into the United States and gender were entered in Block 1, and schools (dummy coded, i.e., SchoolDummy1, SchoolDummy2, SchoolDummy3 and SchoolDummy4) were entered in Block 2. Results are found in Table 5.

Somali Acculturation Index: In the first step age, time since first entry into the United States, and gender accounted for small but significant (Cohen, 1988, 1992) amount of unique variance in Somali Acculturation Index $\left(R^{2}=.08, F(3,94)\right.$ $=2.89, p=.04)$. In the second step, school was found to explain large significant additional variance in the Somali Acculturation Index scores after controlling for age, time in the US, and gender $\left(R^{2}=.36, R^{2} \Delta=.28, F(4,90)=9.75, p=.000\right)$. Age in months significantly contributed to the overall Somali Acculturation Index, predicting that with increasing age, the students tended to have higher levels of retention for their Somali culture. Length of time in the United States also 
significantly contributed to the overall SAI, predicting that with increasing length of time in the U.S., the students tend to have lower levels of overall retention of Somali culture. When looking at the school variables, being a student at School E predicted significantly higher scores on the overall SAI. This suggests that School E students had lower overall Somali culture retention in comparison to the students from the other schools. Also, School D students had significantly lower SAl scores, suggesting that these students had high overall levels of retention of Somali culture compared to students from the other schools.

Somali Language Acculturation: In the first step age, time since first entry into the United States, and gender accounted for marginally significant unique variance in Somali Language Acculturation $\left(R^{2}=.07, F(3,97)=2.54, p=.06\right)$. Time since first entry into the United States was found to be a significant predictor, with increased time in the United States predicting high scores on the SLA suggesting lower Somali language competence. In the second step, school was found to explain moderately significant (Cohen, 1988, 1992) additional variance in the Somali Language Acculturation scores after controlling for age, time in the US, and gender $\left(R^{2}=.20, R^{2} \Delta=.13, F(4,93)=3.67, p=.008\right)$. Further examination of the coefficients revealed that although school variable as a whole was found to explain a significant variance, individually, none of the schools were found to significantly contribute to the students' overall SLA scores.

Somali Identity Acculturation: In the first step age, time since first entry into the United States, and gender did not account for significant amount of variance in Somali Identity Acculturation $\left(R^{2}=.04, F(3,96)=1.34, p=.27\right)$. In 
the second step, school was found to explain moderately significant (Cohen, $1988,1992)$ unique variance in the Somali Identity Acculturation scores after controlling the variance accounted by age, time in the US, and gender $\left(R^{2}=.15\right.$, $\left.R^{2} \Delta=.11, F(4,92)=2.98, p=.02\right)$. Further examination of the coefficients revealed that although school variable as a whole was found to explain a significant variance, individually, none of the schools were found to significantly contribute to the students' overall SIA scores.

Somali Behavior Acculturation: In the first step age, time since first entry into the United States, and gender accounted for marginally significant unique variance in Somali Behavior Acculturation $\left(R^{2}=.07, F(3,95)=2.54, p=.06\right)$. Time since first entry into the United States was found to be a significant predictor, with increased time in the United States predicting high scores on SBA, i.e., lower Somali behavioral acculturation. In the second step, school was found to explain considerably significant additional variance (Cohen, 1988, 1992) in the Somali Behavior Acculturation scores after controlling for age, time in the US, and gender $\left(R^{2}=.41, R^{2} \Lambda=.34, F(4,91)=12.91, p=.000\right)$.

When looking at the school variables, being a student at School E predicted significantly higher scores on the SBA in comparison to the students from the other schools. This suggests that School E students had lower Somali behavior retention in comparison to the students from the other schools. Further, students from School C and D had significantly lower scores on SBA in comparison to School $\mathrm{A}$ and $\mathrm{E}$ and $(\beta=-.28$ and -.33 respectively). This suggests 
that School C and D students had higher Somali behavior retention in comparison to students from School A and B and especially those from School E.

Table 5

Regression Analyses of Demographic Variables on Composite Somali Acculturation (SAl) as well as the Language, Identity and Behavioral Dimensions of Somali Acculturation

\begin{tabular}{|c|c|c|c|c|c|}
\hline \multirow[b]{2}{*}{ Independent Variables } & SAI & \multicolumn{2}{|c|}{ SLA } & $\underline{\text { SIA }}$ & SBA \\
\hline & $\bar{B} \beta$ & $B$ & $\beta$ & $\overline{B \quad} \beta$ & $\bar{B} \beta$ \\
\hline \multicolumn{6}{|l|}{ BLOCK 1} \\
\hline Age in Months & $-.00-.20^{*}$ & -.00 & -.12 & $-.00-.17$ & $-.00-.14$ \\
\hline Time Since First Entry into US & $.01 .23^{*}$ & .01 & $.26^{\star \star}$ & $.01 \quad .13$ & $.01 .24^{\star \star}$ \\
\hline Gender & $-.04-.08$ & -.02 & -.04 & $-.03-.03$ & $-.06-.09$ \\
\hline $\begin{array}{l}R^{2} \\
\text { BLOCK } 2\end{array}$ & $.08^{*}$ & & 07 & .04 & .07 \\
\hline SchoolDummy 1 & $-.09-.12$ & -.05 & -.08 & $-.07-.07$ & $-.11-.11$ \\
\hline SchoolDummy2 & $-.15-.24$ & -.10 & -.18 & $-.12-.13$ & $-.22-.28^{*}$ \\
\hline SchoolDummy3 & $-.17-.27^{*}$ & -.07 & -.13 & $-.13-.14$ & $-.28-.33^{\star *}$ \\
\hline SchoolDummy4 & $.18 \quad .29^{\star}$ & .12 & .21 & $.18 \quad .21$ & $.24 .30^{\star}$ \\
\hline$\Delta R^{2}$ & $.28^{\star \star \star}$ & $.13^{\star *}$ & & $.11^{\star}$ & $.34^{\star \star \star}$ \\
\hline$R^{2}$ & $.36^{\star \star \star}$ & $.20^{\star *}$ & & $.15^{\star}$ & $.41^{* \star *}$ \\
\hline
\end{tabular}

Contributors to School Outcomes

Research Q3 - To what extent does contribution of American acculturation to school adaptation differ from contribution of retaining Somali culture to school adaptation (School grades on Social Behavior and Work Study Habits, SDQ Total Difficulties score, and Proficiency Level Composite scores) based on various demographic variables like school, age, time since first entry into the United States and gender? 
To assess which variables predicted school outcomes, based on a review of the literature and guided by theory, a series of hierarchical regressions were run to predict School Grades (for Social Behavior and Work Study Habits), SDQ Total Difficulties and Proficiency Level Composites. Demographic variables of age in months, time since first entry into the United States and gender were entered in Block 1, schools (dummy coded, i.e., SchoolDummy1, SchoolDummy2, SchoolDummy3 and SchoolDummy4) were entered in Block 2, and acculturation were entered in Block 3. To probe acculturation at a more differentiated level, Block 3 included the scores on the three distinct aspects of the LIB (Language, Identity, and Behavior) for American and Somali Acculturation. Results are found in Table 6 and 7.

Total Difficulties using American Acculturation: In the first step age, time since first entry into the United States, and gender accounted for small significant (Cohen, 1988, 1992) unique variance in Total Difficulties $\left(R^{2}=.08, F(3,92)=\right.$ $2.70, p=.05)$. An examination of the coefficients indicated that gender was found to significantly contribute to the Total Difficulties score $(\beta=-.31, p<.01)$, with female students having lesser behavior problems/difficulties than their male counterparts. In the second step, school was found to explain moderately significant (Cohen, 1988, 1992) additional variance in the Total Difficulties scores after controlling for age, time in the US, and gender $\left(R^{2}=.25, \Delta R^{2}=.17, F(4,88)\right.$ $=4.94, p=.001)$. Table 6 indicates that students from School D scored significantly higher than those from the other schools on the SDQ Difficulties ( $\beta=$ $.41, p<.01)$, i.e., were identified as having greater behavior problems/ difficulties 
by their ESL teacher in comparison to students from the other schools. In the final step, American Acculturation (language, identity and behavior) did not account for significant additional variance in the Total Difficulties scores after controlling the variance explained by age, time in the United States gender, and $\operatorname{school}\left(R^{2}=.26, \Delta R^{2}=.01, F(3,85)=.42, p=.74\right)$.

Social Behavior Grades using American Acculturation: In the first step age, time since first entry into the United States, and gender did not account for any significant unique variance in Social Behavior Grades $\left(R^{2}=.05, F(3,58)=\right.$ $1.13, p=.35)$. In the second step, although school was also not found to explain any significant variance in the Social Behavior Grades after controlling for age, time in the US, and gender $\left(R^{2}=.18, \Delta R^{2}=.12, F(4,54)=1.97, p=.11\right)$, the School D students were found to have significantly lower grades on Social Behavior compared to students from the other schools $(\beta=.42, p<.05)$. In the final step, American Acculturation (language, identity and behavior) did not account for significant additional variance in the Social Behavior Grades after controlling for by age, time in the United States gender, and school $\left(R^{2}=.18, \Delta R^{2}\right.$ $=.01, F(3,51)=.19, p=.90)$.

Work Study Habits Grades using American Acculturation: In the first step age, time since first entry into the United States, and gender did not account for any significant unique variance in Social Behavior Grades $\left(R^{2}=.04, F(3,55)=\right.$ $.80, p=.50)$. In the second step, school was also not found to explain any significant variance in the Social Behavior Grades after controlling for age, time in the US, and gender $\left(R^{2}=.14, \Delta R^{2}=.10, F(4,51)=145, p=.23\right)$. In the final 
step, American Acculturation (language, identity and behavior) also did not account for significant additional variance in the Social Behavior Grades after controlling for age, time in the United States gender, and school $\left(R^{2}=.16, \Delta R^{2}=\right.$ .02, $F(3,48)=.39, p=.72)$.

Proficiency Level Composites for 06-07 using American Acculturation: In the first step age, time since first entry into the United States, and gender accounted for moderately significant (Cohen, 1988, 1992) unique variance in Proficiency Level Composite $\left(R^{2}=.29, F(3,80)=10.74, p=.000\right)$. Age and time since first entry into the United States were found to be significant contributors for the Proficiency Level Composite scores, suggesting that older students and those who have been in the United States longer were inclined to have greater overall proficiency levels as assessed by the ESL ACCESS test $(\beta=.36, p<.01$; $\beta=.28, p<.01$ respectively). In the second step, school was found to explain marginally significant additional variance in the Proficiency Level Composite scores after controlling for age, time in the US, and gender $\left(R^{2}=.36, \Delta R^{2}=.08\right.$, $F(4,76)=2.23, p=.07)$. Further examination of the coefficients indicated that School B students' Proficiency level Composites were significantly lower than students in the other schools. In the final step, American Acculturation (language, identity and behavior) did not account for significant additional variance in the Proficiency Level Composite scores after controlling the variance explained by age, time in the United States gender, and school $\left(R^{2}=.28, \Delta R^{2}=.02, F(3,73)=\right.$ $.59, p=.62)$. 
Finally, when contributions of distinct dimensions of American

acculturation were examined (see Table 6), American language, identity and behavior were not found to be significant contributors for any of the school outcomes.

Table 6

Hierarchical Regression Analyses to predict School Outcomes using Language, Identity, and Behavioral Dimensions of American Acculturation.

\begin{tabular}{|c|c|c|c|c|c|c|}
\hline \multirow[b]{2}{*}{ Independent Variables } & \multicolumn{2}{|c|}{ SDQ-TD } & \multicolumn{2}{|c|}{ G-SB } & G-WSH & PLC \\
\hline & $B$ & $\beta$ & $B$ & $\beta$ & $\overline{B \quad \beta}$ & $B \quad \beta$ \\
\hline \multicolumn{7}{|l|}{ BLOCK 1} \\
\hline Age in Months & -.02 & -.06 & -.01 & -.13 & $-.00-.09$ & $.01 .36^{\star \star \star}$ \\
\hline Time since First Entry into US & -.02 & -.03 & -.01 & -.06 & $-.03-.22$ & $.02 .28^{\star *}$ \\
\hline Gender & -4.31 & $-.31^{\star * \star}$ & ${ }^{*}-.60$ & -.26 & $-.36-.15$ & .18 .12 \\
\hline$R^{2}$ & \multicolumn{2}{|c|}{$.08^{*}$} & \multicolumn{2}{|c|}{.05} & .04 & $.29^{\star \star \star}$ \\
\hline \multicolumn{7}{|l|}{ BLOCK 2} \\
\hline SchoolDummy1 & -.51 & -.03 & .38 & 11 & $-.97-.28$ & $-.69-.32^{*}$ \\
\hline SchoolDummy2 & 4.53 & .28 & .90 & .33 & $.37 \quad .13$ & $-.31-.18$ \\
\hline SchoolDummy3 & 6.99 & $.41^{* *}$ & 1.21 & $.42^{*}$ & $.26 \quad .10$ & $.10 \quad .05$ \\
\hline SchoolDummy4 & 1.25 & -.08 & .18 & .07 & $-.23-.09$ & $-.14-.09$ \\
\hline$\Delta R^{2}$ & \multicolumn{2}{|c|}{$.17^{* * *}$} & \multicolumn{2}{|c|}{.12} & .10 & .07 \\
\hline$R^{2}$ & \multicolumn{2}{|c|}{$.25^{\star \star \star}$} & \multicolumn{2}{|c|}{.18} & .14 & .36 \\
\hline \multicolumn{7}{|l|}{ BLOCK 2} \\
\hline ALA & -1.55 & -.08 & .02 & .01 & 14 & .12 .06 \\
\hline AIA & .01 & .00 & -.02 & -.01 & .13 .09 & .07 .08 \\
\hline $\mathrm{ABA}$ & -.97 & .04 & .38 & .10 & $-.10-.13$ & $-.38-.16$ \\
\hline$\Delta R^{2}$ & \multicolumn{2}{|c|}{.01} & \multicolumn{2}{|c|}{.01} & .02 & .02 \\
\hline$R^{2}$ & \multicolumn{2}{|c|}{.26} & \multicolumn{2}{|c|}{18} & .16 & .38 \\
\hline
\end{tabular}

Total Difficulties using Somali Acculturation: In the first step age, time since first entry into the United States, and gender accounted for significant unique variance in the Total Difficulties scores $\left(R^{2}=.08, F(3,95)=2.79, p=\right.$ 
.045). Similar to the results found in the previous analyses, gender was found to significantly contribute to the Total Difficulties score $(\beta=-.32, p<.01)$, with female students having lesser behavior problems/difficulties than their male counterparts. In the second step, school was found to explain significant additional variance in the Total Difficulties scores after controlling for age, time in the US, and gender $\left(R^{2}=.25, \Delta R^{2}=.17, F(4,91)=5.11, p=.001\right)$. School D students were found to have significantly higher scores on the SDQ Total Difficulties scale suggesting students from this school appeared to have greater behavior problems/ difficulties as rated by their ESL teacher in comparison to students from the other schools $(\beta=.40, p<.05)$. In the final step, Somali Acculturation (language, identity and behavior) did not account for significant additional variance in the Total Difficulties scores after controlling for age, time in the United States gender, and school $\left(R^{2}=.26, \Delta R^{2}=.01, F(3,88)=.45, p=.72\right)$.

Social Behavior Grade using Somali Acculturation: In the first step age, time since first entry into the United States, and gender accounted for marginally significant unique variance in Social Behavior Grades $\left(R^{2}=.06, F(3,59)=1.15\right.$, $p=.34)$. Gender was found to significantly contribute to the grades on social behavior $(\beta=-.27, p<.05)$, suggesting that female students had better grades on social behavior than their male counterparts. In the second step, school was not found to explain any additional significant variance in the Social Behavior Grades after controlling for age, time in the US, and gender $\left(R^{2}=.12, \Delta R^{2}=.08, F(4,55)\right.$ $=2.00, p=.11)$. In the final step, Somali Acculturation (language, identity and behavior) also did not account for significant additional variance in the Social 
Behavior Grades after controlling for age, time in the United States gender, and school $\left(R^{2}=.22, \Delta R^{2}=.05, F(3,52)=1.05, p=.38\right)$.

Work Study Habits Grade using Somali Acculturation: In the first step age, time since first entry into the United States, and gender did not account for any significant unique variance in Work Study Habits Grades $\left(R^{2}=.04, F(3,56)=\right.$ $1.15, p=.81)$. In the second step, school was also not found to explain any significant variance in the Work Study Habits Grades after controlling for age, time in the US, and gender $\left(R^{2}=.14, \Delta R^{2}=.10, F(4,52)=1.48, p=.22\right)$. In the final step, Somali Acculturation (language, identity and behavior) also did not account for significant additional variance in the Work Study Habits Grades after controlling for age, time in the United States gender, and school $\left(R^{2}=.16, \Delta R^{2}=\right.$ $.02, F(3,49)=1.05, p=.71)$.

Proficiency Level Composites for 06-07 using Somali Acculturation: In the first step age, time since first entry into the United States, and gender accounted for significant variance in Proficiency Level Composites $\left(R^{2}=.29, F(3,83)=\right.$ $11.14, p=.00)$. Age and time since first entry into the United States were found to be significant contributors for the Proficiency Level Composite scores, suggesting that older students and those who have been in the United States longer were inclined to have greater overall proficiency levels as assessed by the ACCESS for ELLS test $(\beta=.34, p<.001 ; \beta=.30, p<.001$ respectively). In the second step, school was found to explain marginally significant variance in the Proficiency Level Composite scores after controlling for age, time in the US, and gender $\left(R^{2}=.36, \Delta R^{2}=.08, F(4,79)=2.32, p=.06\right)$. Table 7 indicates that 
students from School B had significantly lower Proficiency Level Composite scores in comparison to the students from the other schools $(\beta=-.28, p<.05)$. In the final step, Somali Acculturation (language, identity and behavior) did not account for significant additional variance in the Proficiency Level Composites after controlling the variance explained by age, time in the United States gender, and school $\left(R^{2}=.41, \Delta R^{2}=.04, F(3,76)=1.83, p=.15\right)$.

Finally, when contributions of distinct dimensions of Somali acculturation were examined (see Table 7), Somali language, identity and behavior were not found to be significant contributors for any of the school outcomes.

Table 7

Hierarchical Regression Analyses to predict School Outcomes using Language, Identity, and Behavioral Dimensions of Somali Acculturation.

\begin{tabular}{|c|c|c|c|c|c|c|c|}
\hline \multirow[b]{2}{*}{ Independent Variables } & \multicolumn{2}{|c|}{ SDQ-TD } & \multicolumn{2}{|c|}{ G-SB } & \multicolumn{2}{|c|}{ G-WSH } & PLC \\
\hline & $\bar{B}$ & $\beta$ & $\bar{B}$ & $\beta$ & $\bar{B}$ & $\bar{\beta}$ & $B \quad \beta$ \\
\hline \multicolumn{8}{|l|}{ BLOCK 1} \\
\hline Age in Months & -.01 & -.04 & -.01 & -.13 & -.01 & -.11 & $.01 .34^{\star \star \star}$ \\
\hline Time since First Entry into US & -.01 & -.01 & -.01 & -.06 & -.03 & -.18 & $.03 .30^{\star \star}$ \\
\hline Gender & -4.38 & $-.32^{\star \star}$ & ${ }^{\star}-.63$ & $-27^{\star}$ & -.37 & -.15 & .18 .12 \\
\hline$R^{2}$ & \multirow{2}{*}{\multicolumn{2}{|c|}{$.08^{*}$}} & \multicolumn{2}{|c|}{.06} & \multirow{2}{*}{\multicolumn{2}{|c|}{.04}} & \multirow[t]{2}{*}{$.29^{\star \star \star}$} \\
\hline \multicolumn{3}{|l|}{ BLOCK 2} & & & & & \\
\hline SchoolDummy1 & -.56 & -.03 & .29 & .09 & -.87 & -.25 & $-.60-.28^{\star}$ \\
\hline SchoolDummy2 & 4.21 & .26 & .82 & .30 & .34 & .12 & $-.32-.18$ \\
\hline SchoolDummy3 & 6.75 & $.40^{\star \star \star}$ & 1.08 & .38 & .08 & .03 & $.01 \quad .01$ \\
\hline SchoolDummy4 & 1.07 & .07 & .20 & .08 & -.01 & -.01 & $-.04-.02$ \\
\hline$\Delta R^{2}$ & \multicolumn{2}{|c|}{$.17^{\star \star \star}$} & \multicolumn{2}{|c|}{.12} & \multicolumn{2}{|c|}{.10} & .08 \\
\hline$R^{2}$ & \multirow{2}{*}{\multicolumn{2}{|c|}{$.25^{\star \star \star}$}} & \multirow{2}{*}{\multicolumn{2}{|c|}{.18}} & \multirow{2}{*}{\multicolumn{2}{|c|}{.14}} & .36 \\
\hline \multicolumn{2}{|l|}{ BLOCK 2} & & & & & & \\
\hline SLA & 1.16 & .04 & .48 & .10 & -.27 & -.05 & .60 .19 \\
\hline SIA & .88 & .05 & .53 & .18 & .53 & .17 & $-.33-.17$ \\
\hline SBA & -2.76 & -.14 & -.61 & -.18 & -.50 & -.15 & $-.39-.18$ \\
\hline$\Delta R^{2}$ & \multicolumn{2}{|c|}{.01} & \multicolumn{2}{|c|}{.05} & \multicolumn{2}{|c|}{.02} & .04 \\
\hline$R^{2}$ & \multicolumn{2}{|c|}{.26} & \multicolumn{2}{|c|}{.22} & \multicolumn{2}{|c|}{.16} & .41 \\
\hline
\end{tabular}


${ }^{\star} p<0.05 ;{ }^{\star \star} p<0.01 ;{ }^{* \star \star} p<.001$

Qualitative Analysis

As part of a mixed methods study, qualitative data were gathered through semi-structured interviews with various professionals including, class teachers as well as ESL teachers, school principals, ESL academic advisor, school counselor as well as community leaders. First, a brief summary of teacher comments will be presented along with any particular consistencies and inconsistencies with the quantitative findings, especially relating to the results on the Strengths and Difficulties Questionnaire (SDQ) which was completed by the ESL teachers. Next, additional information obtained through the interviews to supplement other quantitative findings in general will be presented and discussed.

Teacher comments on school reports cards as well as the comments that the ESL teachers included when completing the SDQ on each of their Somali Bantu students created several consistent themes. Although the SDQ score used for the mail analysis was a continuous variable, for the purpose of interpreting symptoms scores and defining caseness, these scores can also be classified into 3 categories - "normal" to "borderline" to "abnormal" bands for Peer Relationships, Conduct Problems, Emotional Problems, Hyperactivity and Impulsivity and Pro-social Behaviors. The author notes that about $10 \%$ of a community sample score in the "abnormal" range and an additional $10 \%$ score in the borderline range, with some variations in these proportions based on country, age and gender differences (Goodman, 2001). When frequencies for each of the 
5 categories were calculated for the sample, it presented with some interesting findings that were consistent with some of the themes in the qualitative data. A summary of these findings is presented below.

Based on the SDQ scores, 87 percent of the students fell in the normal range, 4 percent in the borderline range and 3 percent fell in the abnormal range for peer relationships. One of the themes based on teacher comments was that these students have a strong desire to fit in. This seems consistent with the finding that a large proportion of these students fell within the normal range for peer relationships, which may be a positive indicator of them trying to fit in with the rests of their peers.

Based on the SDQ scores, 62 percent of the students fell in the normal range, 9 percent in the borderline range and 26 percent fell in the abnormal range for conduct problems. The number falling in the abnormal range appears quite large. The teacher's reported that many of these children have academic and behavior problem; that quite a few struggle with discipline and compliance issues tending to disrespect authority and disregard rules. Some tend to be bullies and others don't care about consequences for their negative behaviors; also the teachers noted that another problem with some of the children was stealing. Thus conduct related problems seem quite prevalent in this population. Although in a related sense, the teachers also discussed that one of the biggest challenges with many of these children has been them getting having difficulty adapting to a formal/ structured classroom environment. This could explain the 
relatively high number of students falling in the abnormal range for conduct problems.

Based on the SDQ scores, 89 percent of the students fell in the normal range, 4 percent in the borderline range and 3 percent fell in the abnormal range for emotional problems. The research literature on refugee children's suggests that often times these children have had very traumatic pasts which then link to them being at risk for emotional problems. The fact that 89 percent of this sample fell in the normal range for emotional problems, suggests that this is a fairly resilient group of refugee children. Some of the themes based on teacher comments were: despite all the challenges these children have undergone in the past, a large number of them have a very strong desire to learn and many have made great academic and behavioral progress over the year; some others words used to describe some of these students were "sweet", "mature", "takes things to stride" and "thrives on positive attention and praise." Again, these findings suggest healthy emotional adjustment. Although most students fell in the healthy range, the teachers also commented on some of the emotional problems they noticed in these children, including - "aggressive when reprimanded," "stressed quite a bit and the resulting physical impact is obvious," "immature in many ways," "suffered lots of PTSD," "undergoes emotional upheavals," and "insecure about current life." Despite these reports, the number of children falling in the concerning categories are minimal, again indicative of this being a rather resilient group. 
Based on the SDQ scores, 67 percent of the students fell in the normal range, 7 percent in the borderline range and 22 percent fell in the abnormal range for hyperactivity/ impulsivity problems. Interestingly, a common theme the teachers reported included: lack of care about consequences, not thinking before speaking or acting, making rash/ impulsive decisions, lacking focus and having difficulty staying still. This behaviors fit the hyperactivity/ impulsivity category; although as previously mentioned, it's possible that some of these hyperactivity/ impulsivity problems are a result of being in a completely new, structured learning environment that may take a little getting used; but in the meanwhile, may feel frustrating and thus the hyperactivity and impulsivity.

Based on the SDQ scores, 55 percent of the students fell in the normal range, 17 percent in the borderline range and 22 percent fell in the abnormal range for prosocial behaviors. The fact that 22 percent of these students fell in the abnormal range, suggesting a lack of prosocial behavior is an interesting finding. Some of the related teacher comments/ reports were: "hurts others feelings," "is manipulative," and "steals." One repeated theme the teachers reported for the ones falling in the normal prosocial behavior range was that many of the children are very helpful and especially love helping their teachers.

Thus, the teacher reports and comments were helpful in further understanding the school adaptation of these children, especially with regard to emotional and behavioral adaptation. Next, relevant information is presented to further understand these children's acculturation related school outcomes. 
One thing that came up very early on in meetings with various professionals working with this population was that in general, the Somali Bantus are a very tight knit community. In fact, to help their children learn/ maintain Somali culture, language and religion, the community leaders have organized weekend school for the children at a community center. Also, several interviewees noted that many children had positive role models like their fathers and older siblings, who try and help and support them in doing well at school. This information is helpful in explaining the finding that in general the children in this sample reported higher levels of overall Somali Acculturation Index as well as Somali Language and Identity Acculturation in comparison to their reported overall American Acculturation Index, American Language and Identity Acculturation scores.

Next, in discussing the school experiences of these children, some of the interviewees noted that amongst the five schools that were included in the study, two schools (School A and School B) are far more integrated in comparison to the others. Further, School $\mathrm{C}$ has been the most challenging in terms of helping integrate the ESL children in general and the Bantu children in particular. Further, Schools C, D, and E were the initial three schools where the Bantus were first enrolled, with School D being particularly inviting of these students and School C having remained somewhat neutral in their engagement all along. An small observation related to integration in the schools was that Schools $A$ and $E$ had ESI classroom walls that were decorated with cultural information on their students from different countries, making it appear very welcoming of diversity, 
appreciating the students' diverse cultural background and allowing non ESL students to learn about their diverse peers. In the context of integration, one of the interviewees reported that the challenge with School C's limited integration lies more in the fact that the school administration has been resistant to integration initiatives, not believing the ESL students have needs that demand special attention. This information even though not quite related to any particular quantitative findings, is valuable in understanding some of the contextual differences in the five schools.

On a slightly different note, another qualitative finding was that students in some of the schools, School $E$ in particular, had greater opportunities to engage in and learn about American culture. The ESL teacher for School E has taken particular interest in these students and frequently rewards their good behavior/ academic performance with an out of school trip like going to the museum, eating at a restaurant, visiting her family for Thanksgiving etc. having such opportunities for culture learning certainly help increase American behavioral acculturation. This seems to relate to the quantitative finding that School E students had significantly lower Somali Behavioral acculturation especially in comparison to Students from Schools C and D.

Finally, School B students were found to have significantly lower English language proficiency scores compared to students at all the other schools. Given that School B's ESL program is the most recently developed program and also given the fact that the length of time in the US for students in School B was significantly lower than the others, helps understand this finding. As was 
previously noted, Proficiency levels depend upon the length of time the students have been in the United States. Given the average student from School B has been in the country for relatively lesser time explains the finding.

Thus the qualitative data helped better understand the quantitative findings of this study, especially in regard to the contextual differences in the schools possibly leading to differing patterns of acculturation and school adaptation for these Somali Bantu refugee children.

A discussion of the results is presented in Chapter V. 


\section{CHAPTER V \\ DISCUSSION AND CONCLUSIONS}

In this chapter I discuss the results and implications of the findings of this study that examined acculturation and school adaptation of Somali Bantu refugee children. In designing this study, I asked the following questions:

(1) To what extent do Somali Bantu children experience American acculturation at the three levels of acculturation, i.e., language, identity, and behavior, when considering demographic variables like age, gender, school, and years in the United States?

(2) To what extent do the Somali children retain their Somali culture at the three levels of acculturation (language, identity and behavior) when considering demographic variables like age, gender, school, and number of years in the United States?

(3) To what extent does the contribution of American acculturation to school adaptation differ from contribution of retaining Somali culture to school adaptation (school grades, ACCESS scores, and SDQ scores) based on various demographic variables like age, gender, school, and years in the US?

First, l'll discuss the findings of the main and supplemental analyses in reference to possible explanations of the findings and their convergence 
divergence with previous research. Next, theoretical and research implications of the study will be discussed. Finally, limitations of the study will be reviewed and suggestions for future directions within psychological expertise research will be made.

\section{Discussion of the Main Results}

The present study tested the relationship between acculturative styles and school adaptation among a group of Somali Bantu refugee children, using a multidimensional model of acculturation (language, identity and behavior) and assessing it independently with respect to both Somali and American cultures.

Paired $t$-tests revealed differences in overall level of American and Somali acculturation ( $A A \mathrm{I}$ and $\mathrm{SAI}$ ), suggesting that as a group, the students were not balanced with respect to their level of American and Somali acculturation. Further paired $t$-tests revealed that overall retention of Somali culture was significantly higher than overall level of American acculturation in this group of students. Somali language competence and identity were also significantly higher than their American counterparts. Lastly, no differences were observed in the group's level of American and Somali behavioral acculturation. Results from prior studies have been mixed, with differing levels of acculturation to host culture and retention of native culture (Trickett \& Birman, 2005).

Next, to explore the relationship between the various demographic variables, acculturation and school outcomes, Pearson product-moment correlation coefficients were calculated. Age was found to be associated with greater overall American acculturation and higher Proficiency Level Composites. 
Time in the country was associated with lower overall level of Somali acculturation. Perhaps not surprisingly, over time, these children appear to be become more "American" and less "Somali" in their general orientations. Female participants were less likely to have behavioral problems/difficulties and also more likely to have better grades on Social Behavior; these were the only gender differences found among any of the variables. In reviewing the qualitative data on teacher's comments regarding different students, triangulating information was obtained. Teachers reported more concerns with behavior problems being indicated for male students, with very few references made for female students regarding behavior problems. This finding was in line with a previous study on the acculturation and school adaptation of Russian refugee adolescents by Trickett and Birman (2005) who that noted female participants were less likely to incur disciplinary infractions.

A moderate correlation was found between overall American acculturation Index and Somali acculturation Index (.45) scores. The magnitude of this correlation suggests the relative ease for these Somali Bantu refugee children in being bicultural within the school context, as an emphasis on one culture does not imply a relative diminishing of the other culture. As such, this may imply that American acculturation at these schools is more of an option than a requirement for these children. In the context of prior literature, this finding suggested an important difference. Contrary to the finding from the current study, Trickett and Birman's (2005) study found a large negative correlation between overall American and overall Russian acculturation among a group of Russian refugee 
adolescents. Those authors concluded that the difference was indicative of the students having difficulty being bicultural at the school. They hypothesized that American acculturation at the school of study was a requirement more than an option. The authors concluded that the differences may be resulting from a certain kind of acculturative press - assimilationist press (pressures exerted on immigrant and refugee students by schools and the surrounding communities to assimilate.) In other words, the school was encouraging American acculturation while discouraging attachment to Russian culture.

Applying the above theory to the current findings would suggest that the schools serving as sites for the current study may be imposing an integrationist press, with equal importance given to the student's retaining their native Somali culture while also accepting values and customs of the American culture. Thus, in light of the literature, this finding may be an overall positive for this group of children given that in several studies integration has been shown to promote the most constructive path toward adjustment to a new culture (Berry, Kim, \& Boski, 1988; Kovacev \& Shute, 2004; Liebkind, 1996; Sam \& Berry, 1995). Another possibility could be that the United States viewed Russia as "the evil empire" for decades, but does not have any such negative perceptions of Somalia, and the teachers may be conveying these differences.

Overall level of Somali cultural retention was associated with better grades on Social Behavior and overall level of American acculturation was correlated with greater behavioral difficulties. Several other studies have reported similar findings, where retention of culture of origin serves as a positive factor in school 
performance. A significantly large correlation ( .70$)$ between grades on Social Behavior and Work Study Habits suggests that a poor grade on one would more than likely result in a poor grade in the other as well.

Next, age and tirne in the country were associated with higher Proficiency Level Composite scores. Age and length of time in the country have been well documented as proxies for measures of acculturation, making this finding in line with the literature. Further, one of the theories on second language acquisition differentiates between two types of languages: basic interpersonal communications skills (BICS) and cognitive academic language proficiency (CALP). Research has shown that the average student can develop conversational fluency within two to five years, but that developing fluency in more technical, academic language can take from four to seven years depending on many variables such as language proficiency level, age and time of arrival at school, level of academic proficiency in native language, and the degree of support for achieving academic proficiency (Cummins, 1981, 1986; Hatuka, Butler, \& Witt, 2000; Thomas \& Collier, 1997). Based on this theory and the variables it outlines as significant contributors to second language acquisition, the findings that age and length of time in the country were associated with higher proficiency level composites for this sample seem appropriate. Especially since the ACCESS for ELLs measures the academic English language proficiency in English language learners.

In comparing the sample demographics across the three components of American acculturation - language, identity and behavior, a statistically 
significant result for the variable school was found across the combined components of American acculturation. When results for the three components of American acculturation were considered separately, the only difference to reach statistical significance was American Language Acculturation. Further inspection of the mean scores indicated that School D students had significantly higher American language competency than School E students. Similarly, when sample demographics were compared across the three components of Somali acculturation, school was again found to be the significantly different variable across the combined components of Somali acculturation. When the results for the three components of Somali acculturation were considered separately, Somali Behavioral Acculturation was the only one to reach statistical significance. Inspection of the mean scores indicated that School E students had significantly lower retention of Somali cultural behaviors compared to School C and D students.

Schools C, D and E have larger Somali student populations than Schools $A$ and $B$. Further, among the five ESL programs, School $E$ is the first one to admit Somali Bantu children and has the largest numbers, with School B being the most recently developed ESL program with very recent admissions of Somali Bantu children. This may mean that School E children may have had more exposure and opportunities within the school setting to interact with American students and familiarize themselves with American culture and thus leading to lower retention of Somali culture. 
Another factor that may be vital in understanding the above finding is whether these students have opportunities outside of the school setting to be exposed to American culture and to engage in American cultural behaviors. Based on the investigator's discussion with the ESL teacher at School E, it appears that she often rewards her students' good behavior and academic progress with outside-of-school activities like trips to the museum and restaurants, along with invitations to her home to have meals with her family on special national holidays like Thanksgiving and Christmas. These opportunities naturally may increase these children's familiarity with American culture and thus simultaneously to a decrease in their retention of Somali culture.

School $E$ is the only school among the five to have an onsite school-based therapist for mental health services. Mental health counseling, being a part of western culture is another service that is more readily available to School E students, making it an additional exposure to western culture, possibly increasing their higher overall American acculturation index scores.

Lastly, no statistically significant differences were found for sample demographics (different schools, age and length of time in the US categories and gender) across the four school outcome variables, i.e., total difficulties, grades on social behavior and work study habits, and proficiency level composites for 200607. This is an interesting finding, given that the students did differ on their levels of acculturation based on the demographic variables.

In summary, a consistently significant finding thus far has been the role of school in the student's American and Somali acculturation. This finding may be 
explained by the literature on the ecological perspective concerning patterns of acculturation. The theory states that because acculturation as a process unfolds within the context of specific communities and represents a transaction between acculturating individuals, groups, and communities, culture and context should be inseparable in the study of the acculturation process (Birman, 1994; Birman et al., 2005; Padilla, 1980; Portes \& Rumbaut, 1990).

Unfortunately, an in-depth understanding of the school context in ways that may have explained further the acculturation patterns found in the data was beyond the scope of this study. Future research should focus more specifically on a deeper understanding of the role of local context, i.e., schools, for purposes of improving the ecological validity (Bronfenbrenner, 1977) of the reported data and its influences on the acculturation process of Somali Bantu refugee children. Contributors to American Acculturation

The first research question investigated the unique variance explained by the four demographic variables - age, time since first entry into the United States, gender and school in predicting overall American acculturation as well as American acculturation at the more differentiated levels - language, identity, and behavior.

The results of the analyses suggest that, among the variables studied, school was the only significant predictor of overall level of American acculturation. This alludes to the previously presented finding that the 5 different schools that were part of the study probably had significant contextual differences in ways such that led to significantly different acculturation outcomes 
for the students. Therefore, while age and length of time in the U.S. have been accepted as frequently used standard proxies for measuring acculturation (Birman \& Trickett, 2005), significance of the school context brings light to another variable that may be contributing to the acculturation of these children. This also suggests that acculturation as a process is reflective of more than just the effects of the passage of time as was noted too in the study on Russian refugee adolescents' school adaptation (Birman \& Trickett, 2005). Lastly, for the purpose of this study, the Language Identity Behavior Acculturation Scale was found to be an extremely useful tool and overall the Chronbach Alpha reliabilities for the overall scale and the subscales held very well.

A number of possible differences may have led to these results. Given that the empirical literature indicates that school is a primary context for refugee children's acculturation and adjustment to the host country, the different cultural learning opportunities a child has available at the school may play a significant role in whether and how much cultural integration may take place. Further exploration of the school context would be essential in better understanding its role in the acculturation process of these children.

With respect to the different aspects of acculturation, age in months was a significant predictor of American language acculturation, i.e., level of English competence, which is similar to prior findings in studies of acculturation. Lastly, school appeared to be a significant predictor for all three aspects of American acculturation - language, identity and behavior. This finding corresponds with the previous research that adopts a general ecological perspective in understanding 
acculturation, emphasizing the importance of the interplay of culture and context (Birman et al., 2005). These results suggest that the overall American acculturation as well as American acculturation at the differentiated levels for these Somali Bantu children depended primarily upon the different schools in which they were enrolled. Further, it is important to note that the children's families did not necessarily have a say in regard to which school their child got enrolled into. The enrollment decision for the year the data was collected was made by the school system based on the racial profile such that there is a balance.

Contributors to Somali Acculturation

The second research question investigated the unique variance explained by the four demographic variables - age, time since first entry into the United States, gender and school in predicting overall Somali acculturation as well as Somali acculturation at the more differentiated levels - language, identity, and behavior.

The results of the analyses suggest that age and school were found to be significant predictors of overall Somali Acculturation Index. These results suggested that older children retained higher levels of overall Somali culture. Also, in looking at the school variable, School E students were found to retain lower overall levels of Somali culture compared to students from the other schools. Age as a significant predictor of overall Somali Acculturation Index makes considerable sense, given that the older children in this sample were entering early adolescence. 
Erikson's (1968) theory of ego idertity formation notes that an achieved identity is the result of a period of exploration and experimentation that typically takes place during adolescence and that leads to a decision or a commitment in various areas such as occupation or religion (Phinney, 1996). Ethric identity formation has been thought of as a process similar to ego identity formation. In an article examining various models of ethnir, identity rormation, Phinney (1989) notes that early adolescents fall in the first stage of etric : Sentity formation where they may have a preterence for this corvi larit s: native culture based on their exposure to ethnic identity i'sues. He stercs hat thece preferences are rot

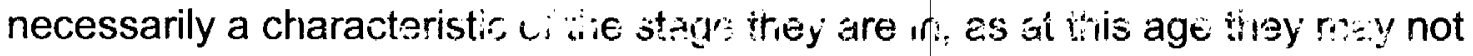

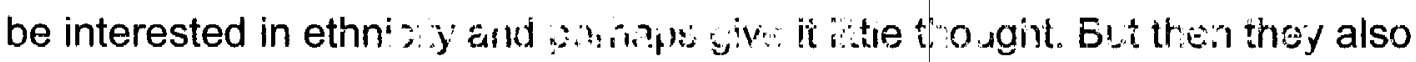
absorb positive ethnic aititudes rom parsents or other ant its ar,d thareicre nay show a preference for their culture, eifir inough they have not thought through the issues themselyes (Phinriəy, 1969). This may explain the current resulis where in the older Somali children tenaed to retain higher leveis of Somali culture in general, given that the Somali community is very tight knit and its community leaders have beeri actively involved in educating their Somali youth about Sornali culture including the Islamic religion, ihrough active participation in weekend school.

It is more likely that the younger chilidren were born in the United States or arrived in the United States at a very young age allowing them fewer personal experiences with life in Somalia or the refugee camps in Africa compared to the older children. Lastly, unilike the Soviet Jews in the acculturation and school 
adjustment study by Birman and Trickett (2005), who upon immigrating to the United States becoming part of the White majority, the experience of these Somali Bantu refugee children involves coming from a previously oppressed minority group in their country of origin to becoming part of the ethnic/ racial minority group in the United States.

With respect to the different aspects of acculturation, length of time in the United States was a significant predictor of Somali Language Acculturation and Somali Behavioral Acculturation. These results suggested that those students who have lived in the United States for a longer time had lower levels of Somali language competence. Further, an increasing length of time in the United States related to lower levels of Somali behavioral acculturation, i.e., predicting a low retention of Somali culture for students who have lived in the United States the longest. One possible explanation for these findings may be that the older children in the sample were entering adolescence, a stage of life where peer relations and fitting in with the majority culture are prevalent issues that may well lead to a loss of home culture and language.

School was also found to be a significant predictor of Somali Behavioral Acculturation. The results suggested that students from School C and School D had higher retention levels of Somali cultural behaviors than did students from School A and School B and especially School E. Contributors to School Outcomes

The third research question, after controlling for the demographic variables, investigated the unique contribution of American and Somali 
acculturation on the four school outcomes - SDQ Total Difficulties scores, Grades on Social Behavior and Work Study Habits and ACCESS for ELLs scores for Proficiency Level Composites for 2006-07.

Contrary to findings from previous studies, the results of the analyses for this sample suggest that neither the overall level of American acculturation or retention of Somali culture or acculturation at the differentiated levels of language, identity and behavior for both American and Somali culture were found to be significant contributors for any of the school outcomes for these refugee children, despite the significant correlations found between acculturation and some of the school outcomes. The inconsistency in these results with the rest of the research literature may be explained by the fact that most empirical literature on acculturation has been on the adolescent population. Given the current study was on elementary age children with a few early adolescents, the tendency for socially desirable responses may be higher, given the acculturation scale was a self report measure. This makes sense, given the other measures including school grades, proficiency level composite scores and total difficulty scores were more independent, objective measures. Young children may be easily susceptible to exaggerating their responses positively or negatively, thereby masking the relationships between acculturation and school outcomes.

Gender was found to significantly predict Total Difficulties scores, females tending to have fewer difficulties than males. Age and length of time in the United States were found to be significant predictors of Proficiency Level Composites scores for 2006-07, indicating that increasing age and length of time in the United 
States related to higher Proficiency Level Composites on the ACCESS for ELLs. School was found to be a significant predictor for Total Difficulties scores as well as grades on Social Behavior. Regarding Total Difficulties, School D students appeared to have greater difficulties in comparison to those from the other schools. School D students also appeared to have significantly lower grades on Social Behavior.

Last, none of the research variables appeared to contribute significantly to the Work Study Habits grades. Given that Grades on Work Study Habits were significantly correlated with grades on Social Behavior and also scores on SDQ's Total Difficulties and since school was a significant predictor for both those variables, one would have expected similar results for grades on Work Study Habits as well. However, that was not the case making it a particularly interesting finding.

Implications for Practice and Future Research

The present study sought to explore the acculturation process and school outcomes of a group of Somali Bantu refugee children. The focus on refugee children who represent an understudied population presents valuable and unique information that adds to the acculturation literature. The findings of this study in context with past research have significant implications. The results of this study clearly demonstrated that the school context was the most significant variable to account for the acculturation and school adaptation of these Somali Bantu children, and that the acculturation and adaptation process is complex and 
different for different groups, varying as a result of differing contextual patterns within the ecology of these individuals. Thus, the study provides evidence that it is important when studying acculturation to account for school community differences that affect the process.

Although I have suggested that the key finding in this study is that the school context is contributing to the reported findings on the acculturation and adaptation process of these children, the study did not include a more in-depth exploration of the school contexts. A far more refined appreciation for the differences and similarities of the five school contexts is needed to further the school context and acculturation and school adaptation linkage. Ethnic density, discrimination, social support, sense of school belonging, the availability of opportunities for cultural maintenance are some of the school context factors that need explicit consideration in furthering an appreciation of the context in which acculturation takes place.

Another important point to note is that there may have been a teacher effect. Further exploration for the schools should include data on teachers and their philosophies as that would allow for a better understanding of what type of school and teacher philosophy better serves these children in regard to their acculturation and school adaptation. Employing volunteers in schools to help with the integration of these students with mainstream American culture while also encouraging them to retain their home culture, may be another way of allowing children equal opportunity for increased exposure to culture there by facilitating their adaptation process. 
A significant element of many refugee children's psychosocial history involves pre- and post-migration stressors, trauma and grief, which also play a significant role in their acculturation and adaptation process in their country of resettlement. Given the limitations of the study, this significant factor was not explored.

Lastly, while school differences accounted for the major findings in this study, the role of the other independent variables including age, length of time in the United States and gender cannot be undermined. These findings relating to the demographic variables were consistent with the empirical literature, thus further reinforcing the importance of considering age, length of time in the US and gender when addressing refugee children's adaptation and acculturation.

Future work should focus on studying these differences in school contexts including whether they are naturally occurring or are a result of an acculturative press on the part of the school system. Qualitative data including parent, teacher, and student interviews as well as classroom observations would further enrich the understanding and appreciation of the complexities of the refugee children's acculturation process.

\section{Limitations}

As with all studies, this study has several limitations. Although the participants were recruited from all the five of the schools that had a Somali Bantu student population, in a Midwestern city, (although there was not complete data on all the variables for all the students), the level of representativeness of the sample of Somali Bantu children in the United States is untested. This is 
particularly relevant, given the findings from the study that indicate that there are many individual, group and contextual variables that may interact together to influence the acculturation and adaptation process for refugees. Notwithstanding, the researcher assumes that the sample used in this study is reasonably representative of the Somali Bantu elementary school-aged refugee children in the particular Midwestern city where the data was collected.

The self-report nature of the acculturation instrument is another limitation. Because of common method variance and the possibility of response set bias, the use of self-report measures tends to inflate the correlations found among variables. Further, when using self-report measures, researchers cannot know how truthfully respondents answer. Social desirability might affect the way participants answer the items.

A final limitation of this study is the limited attention given to other important factors that influence acculturation and adaptation. The role of parentchild relationship, pre-migration stressors, school belonging, metal health problems, residential and school community and locations, parents' socioeconomic status, culture brokering and other important acculturation and adaptation variables were not examined in this study, although they surely play an important part in the ways in which acculturation and adaptation are related.

\section{Conclusion}

The empirical and theoretical literature suggests that acculturation as a process is multidimensional and complex and for that very reason the 
acculturation outcomes for different populations can differ significantly. In fact, this has been reiterated in the findings of this study on Somali Bantu refugee children. The findings suggest that while certain demographic variables like age and length of time in the host country remain quite consistent in their impact on acculturation and adaptation of refugees and immigrants, other contextual factors, school context in this study, play out differently for different groups. This study contributes documentation of the effects school differences on a unique and specific minority: Somali Bantu refugee children. In this context, the current study documents for the first time the acculturation process, from a differentiated multidimensional perspective, of these Somali Bantu refugee children.

Most clearly, the findings document the impact of school differences on these children's acculturation as well as school adaptation process. This finding emphasizes the need for school administrators to attend to the school environment and their initiatives in helping refugee and immigrant children integrate into the larger school system. This would require an effort on the part of the leadership in the school administration from top all the way down to be particularly sensitive to the ongoing needs of refugee children. These findings suggest a greater need for the school administrators to make deliberate efforts re-examine their school policies to better meet the needs of their refugee students. Finally, these findings amplify the need for more ongoing professional development and training of the school administration on refugee children's needs for school success with an effort towards helping integrate these students 
into the larger school systems while respecting their diverse cultural backgrounds. 


\section{REFERENCES}

Alexander, K.L., \& Entwisle, D.R. (1988). Achievement in the first 2 years of school: Patterns and processes. Monographs of the Society for Research in Child Development, 53, (2), Serial No. 218.

Alexander, K.L., Entwisle, D.R., \& Dauber, S.L. (1993). First-grade classroom behavior. Its short and long-term consequences for school performance. Child Development, 64, 801-814.

Allwood, M.A., Bell-Dolan, D., \& Husain, S.A. (2002). Children's trauma and adjustment reactions to violent and nonviolent war experiences. Journal of the American Academy of Child \& Adolescent Psychiatry, 41(4), 450-457.

Almqvist, K., \& Broberg, A.G. (1999). Mental health and social adjustment in young refugee children 31/2 years after their arrival in Sweden. Journal of the American Academy of Child and Adolescent Psychiatry, 38, 723- 730

Asali, Z. (2003). Of Liberty, Security, and the Human Spirit. Paper presented at the conference "Confronting Racial Profiling in the $21^{\text {st }}$ Century: New Challenges and Implications for Racial Justice," Northeastern University, Boston.

Ascher, C. (1985). The social and psychological adjustment of Southeast Asian refugees. Urban Review, 17(2), 147-152. 
Ataka, B., \& Berry, J.W., (2002). Psychological, socio-cultural, and marital adaptation of Turkish immigrant couples in Canada. International Journal of Psychology, 37(1), 13-26.

Bankston III, C. L., \& Zhou, M. (1997). The social adjustment of Vietnamese American adolescents: Evidence for a segmented-assimilation approach. Social Science Quarterly, 78, 508-523.

Bankston III, C. L., \& Zhou, M. (2002). Being well vs. doing well: Self-esteem and school performance among immigrant and nonimmigrant racial and ethnic groups. International Migration Review, 36, 389-415.

Berman, H. (2001). Children and war: Current understandings and future directions. Public Health Nursing, 18(4), 243-252.

Berry, J. W. (1974). Psychological aspects of cultural pluralism. Topics in Culture Learning, 2, 17-22.

Berry, J. W. (1980). Social and cultural change. In H. C. Triandis \& R. W. Brislin (Eds.), Handbook of Cross-Cultural Psychology: Social Psychology (vol. 5, pp. 211-279). Boston: Allyn and Bacon.

Berry, J.W. (1994). An ecological perspective on cultural and ethnic psychology. In E. Trickett (Ed.). Human Diversity: Perspectives on People in Context, (pp. 115 - 141). San Francisco: Jossey-Bass.

Berry, J.W. Kim, U., \& Boski, P. (1988). Psychological acculturation of immigrants. In Y.K. Young, W.B. Gudykunst (Eds.), Cross-Cultural Adaptation: Current Theory and Research (pp. 312-326), Newbury Park, Ca: Sage. 
Berry, J. W., Poortinga, Y. H., Segall, M. H., \& Dasen, P. R. (2002). CrossCultural Psychology: Research and Applications ( $2^{\text {nd }}$ Ed.). Cambridge, UK: Cambridge University Press

Berry, J.W., Trimble, J.E., \& Olmedo, E.L. (1986). Assessment of acculturation. In W.J. Lonner \& J.W. Berry (Eds.), Field Methods in Cross-Cultural Research (Vol. 8, pp. 291-324). Beverly Hills, CA: Sage.

Bhattacharya, G. (2000). The School Adjustment of South Asian Immigrant Children in the United States. Adolescence, 35, 77-85.

Bhui, K., Abdi, A., Abdi, M., Pereira, S., Dualeh, M., et al. (2003). Traumatic events, migration characteristics and psychiatric symptoms among Somali refugees' preliminary communication. Social Psychiatry \& Psychiatric Epidemiology, 38(1), 35-43.

Birman, D. (1994). Acculturation and human diversity in a multicultural society. In E.J. Trickett, R.J. Watts, \& D. Birman (Eds.), Human Diversity: Perspectives on People in Context (pp. 261-284). San Francisco: JosseyBass.

Birman, D. (1998). Biculturalism and perceived competence of Latino immigrant adolescents. American Journal of Community Psychology, 26(3), 335-354.

Birman, D. (2006). Acculturation gap and family adjustment: Findings with Soviet Jewish refugees in the United States and implications for measurement. Journal of Cross-Cultural Psychology, 37(5), 568-589. 
Birman, D., \& Trickett, E.J. (1999). Cultural transitions in first-generation immigrants: Acculturation of Soviet Jewish refugee adolescents and parents. Journal of Cross-Cultural Psychology, 32(4), 456-477.

Birman, D., \& Trickett, E.J. (2001). Cultural transitions in first-generation immigrants: acculturation of Soviet Jewish refugee adolescents and parents. Journal of Cross-Cultural Psychology, 32, 456-477.

Birman, D., Trickett, E.J., \& Bacchus, N. (2001). Somali Refugee Youth in Maryland: A Needs Assessment (Maryland Office for New Americans), Maryland Department of Human resources). Retrieved April 22, 2007, from http://63.26.98.116/mona/pdf/somali.pdf

Birman, D., Trickett, E.J., \& Buchanan, R.M. (2005). A tale of two cities: Replication of a study on the acculturation and adaptation of immigrant adolescents from the Former Soviet Union in a different community context. American Journal of Community Psychology, 35(1/2), 83-101.

Birman, D., Trickett, E.J., \& Vinokurov, A. (2002). Acculturation and adaptation of Soviet Jewish Refugee Adolescents: Predictors of Adjustment across Life Domains. American Journal of Community Psychology, 30(5), 585607.

Birman, D., \& Tyler, E.B. (1994). Acculturation and alienation of Soviet Jewish refugees in the United States. Genetic, Social \& General psychology Monographs, 120, 101-115.

Boothby, N. (1994). Trauma and violence among refugee children. In A.J. Marsella, T. Bornmann, S. Ekblad \& J. Orley (Eds.), Amidst Peril and Pain: 
The Mental Health and Well-Being of the World's Refugees (pp. 239-29).

Washington, D.C.: American psychological Association.

Boyd, W.L., \& Shouse, R.C. (1997). The problems and promise of urban

schools. In, H.J. Walberg, O. Reyes, \& R. Weissberg (Eds.), Children and Youth: Interdisciplinary Perspectives Issues in Children's and Families' Lives (Vol. 7, pp. 141-165). Thousand Oaks, CA: Sage Publications. Boyden, J., de Berry, J., Feeny, T., \& Hart, J. (2002). Children Affected by Conflict in South Asia: A Review of Trends and Issues Identified Through Secondary Research. Refugee Studies Center, RSC Working Paper No. 7, University of Oxford. Retrieved April 22, 2007, from http://rsc.ox.ac.uk/PDFs/workingpaper7.pdf

Bozorgmehr, M., \& Sabagh, G. (1991). Iranian exiles and immigrants in Los Angeles. In A. Fathi (Ed.), Iranian Refugees and Exiles since Khomeini (pp. 121-160). Costa Mesa, CA: Mazda Publishers.

Bradbury, M. (1997). Somaliland: Country Report. Islington, London: Catholic Institute for International Relations.

Brislin, R.W. (1970). Back-translation for cross cultural research. Journal of Cross Cultural Psychology, 1, 185-216.

Brislin, R.W. (2000). Back-translation. In A.E. Kazdin (Ed.), Encyclopedia of Psychology, Vol. 1. (pp. 359-360). Washington, DC, US; New York, NY, US: American Psychological Association; Oxford University Press.

Bronfenbrenner, U. (1977). Toward an experimental ecology of human development. American Psychologist, 32, 513-530. 
Caplan, N., Choy, M.H., \& Whitmore, J.K. (1994). Children of the Boat People: A Study of Educational Success. Ann Arbor: University of Michigan Press.

Caplan, N., Whitmore, J.K., \& Choy, M.H. (1989). The Boat People and Achievement in America: A Study of Family Life, Hardwork, and Cultural Values. Ann Arbor: University of Michigan Press.

Carter, R.B. (1999). Counseling Muslim children in school settings. Professional School Counseling, 2, 183-188.

Cha, E. S., Kim, K. H., \& Erlen, J. A. (2007). Translation of scales in crosscultural research: issues and techniques. Journal of Advanced Nursing, 58(4), 386-395.

Cheng, L.L. (1998). Enhancing the communication skills of newly-arrived Asians American students. ERIC Clearinghouse on Urban Education, 36, ISSN 08898049.

Cohen, J. (1988). Statistical Power Analysis for the Behavioral Sciences (2nd edition). Hillsdale, NJ: Erlbaum.

Cohen, J. (1992). A power primer. Psychological Bulletin, 112, 155-159.

Collier, V. (1987). Age and rate of acquisition of second language for academic purposes. TESOL Quarterly, 21(4), 617-641.

Cortes, D.E., Rogler, L.H., \& Malgady, R.G. (1994). Biculturality among Puerto Rican adults in the United States. American Journal of Community Psychology, 22(5), 707-721.

Cowart, M.T., \& Cowart, R.E., (1993). Southeast Asian refugee youth and the cycle of violence. NASSP Bulletin, 77 (557), 41-45. 
Cowart, M.T., \& Cowart, R.E. (2002). Invisible No More: Including the Refugee and Immigrant Experience in the Curriculum. Paper presented at the annual meeting of the National Association of Multicultural Education, Washington, DC.

Crane, J. (1996). Effects of home environment, SES, and maternal test scores on mathematics achievement. Journal of Educational Research, 89, 305314.

Cummins, J. (1981). Empirical and theoretical underpinnings of bilingual education. Journal of Education, 163, 16-29.

Cummins, J. (1986). Empowering minority students: A framework for intervention. Harvard Educational Review, 56, 18-36.

Cummins, J. (1992). Bilingual education and English immersion: The Ramirez report in theoretical perspective. Bilingual Research Journal, 16, 91-104.

Cummins, J. (2000). Language, Power, and Pedagogy: Bilingual Children in the Crossfire. Clevedon, England: Multilingual Matters.

Cutts, M., (Ed.), (2000). The State of the World's Refugees: 50 Years of Humanitarian Action. Oxford, UK: Oxford University Press.

Delgado-Gaitan, C. (1994). Russian refugee families: Accommodating aspirations through education. Anthropology \& Educational Quarterly, 25(2), 137-155.

Derwing, T. M., DeCorby, E., Ichikawa, J., \& Jamieson, K. (1999). Some factors that affect the success of ESL high school students. The Canadian Modern Language Review, 55, 532-547. 
Duncan J. (2000). Overview of Mental Health Findings for UAM and Separated Children Interviewed as part of UNHCR Best Interest Determinations. Kakuma Refugee Camp, Kenya: Unpublished Report.

Eisenbruch, M. (1988). The mental health of refugee children and their cultural development. International Migration Review, 22, 282-300.

Eisenbruch, M. (1991). From post-traumatic stress disorder to cultural bereavement: Diagnosis of Southeast Asian refugees. Social Science \& Medicine, 33(6), 673680.

Eisikovits, R.A. (1995). Educational success and long-term adaptation of immigrant students in Israel. Qualitative Studies in Education, 8, 171-181.

Ensminger, M.E., \& Slusarick, A.L. (1992). Paths to high school graduation or dropout: A longitudinal study of a first-grade cohort. Sociology of Education, 65, 95-113.

Erikson, E. H. (1968). Identity. New York: Norton.

Feldman, S.S., \& Wentzel, K.R. (1990). Relations among family interaction patterns, classroom self-restraint, and academic achievement in preadolescent boys. Journal of Educational Psychology, 82, 813-819.

Fernández-Kelly, P., \& Curran, S. (2001). Nicaraguans: Voices lost, voices found. In R. Rumbaut \& A. Portes (Eds.), Ethnicities: Children of Immigrants in America (pp. 127-155). Berkeley: University of California Press.

Fisher, C. B., Wallace, S. A., \& Fenton, R. E. (2000). Discrimination distress during adolescence. Journal of Youth and Adolescence, 29, 679-695. 
Fox, P.G., Cowell, J.M., \& Montgomery, A.C. (1994). The effects of violence on health and adjustment of Southeast Asian refugee children: An integrative review. Public Health Nursing, 11(3), 195-201.

French, D. C., \& Conrad, J. (2001). School dropout as predicted by peer rejection and antisocial behavior. Journal of Research on Adolescence, $11,225-244$.

Gall, M. D., Borg, W. R., \& Gall, J. P. (1996). Educational Research: An Introduction. White Plains, NY: Longman.

Garnett, B., \& Ungerleider, C. (2008). An Introductory Look at the Academic Trajectories of ESL Students. Working Paper Series. Retrieved July 8, 2008 from http://riim.metropolis.net/Virtual\%20Library/2008MP08-02.pdf

Gibson, M. (1988). Accommodation without Assimilation: Punjabi Sikh Immigrants in an American High School. Ithaca, NY: Cornell University Press.

Gibson, M. A. (1998). Promoting academic success among immigrant students: Is acculturation the issue? Educational Policy, 12, 615-633.

Goodman, R. (1997). The Strengths and Difficulties Questionnaire: A Research Note. Journal of Child Psychology and Psychiatry, 38, 581-586.

Goodman R (2001) Psychometric properties of the Strengths and Difficulties Questionnaire (SDQ). Journal of the American Academy of Child and Adolescent Psychiatry, 40, 1337-1345.

Gordon, M. (1964). Assimilation in American Life: The Role of Race, Religion, and National Origins. New York: Oxford University Press. 
Gorges, T.C., \& Elliott, S.N. (1995). Homework: Parent and student involvement and their effects on academic achievement. Canadian Journal of School Psychology, 11, 18-31.

Gottlieb, M., Cranley, E.M., \& Oliver, A.R. (2007). Understanding the WIDA English Language Proficiency Standards. A Resource Guide. Retrieved July 8, 2008 from http://www.wida.us/standards/Resource Guide web.pdf.

Grolnick, W.S., \& Ryan, R.M. (1989). Parent styles associated with children's self regulation and competence in school. Journal of Educational Psychology, 81, 143-154.

Hakuta, K., Butler, Y.G., \& Witt, D. (2000). How Long Does it Take English Learners to Attain Proficiency? Santa Barbara, CA: University of California, Linguistic Minority Research Inst.

Halcon, L.L., Roberston, C.L., Savik, K., Johnson, D.R., Spring, M.A., Butcher, J.N., Westermeyer, J.J., \& Jaranson, J.M. (2004). Trauma and coping in Somali and Oromo refugee youth. Journal of Adolescent Health, 35, 1725.

Harrell-Bond, B. (2000). Are Refugee Camps Good for Children? (Working Paper 29). Cairo, Egypt: American University in Cairo, Forced Migration and Refugee Studies.

Holtzman, W., \& Bornemann, T., (Eds). (1990). Mental Health of Immigrants and Refugees. Austin, TX, US: Hogg Foundation for Mental Health. 
Hones, D.F. (2002). American Dreams, Global Visions: Dialogic Teacher Research with Refugee and Immigrant Families. Mahwah, NJ: Erlbaum. Hones, D.S., \& Cha, C.S. (1999). Educating New Americans: Immigrant Lives and Learning. Mahwah, NJ: Erlbaum.

House, J. D. (2001). The relationship between academic self-concept and school withdrawal. Journal of Social Psychology, 133, 125-127.

Huang, J. (2000) Integration of academic content learning and academic literacy skills development of L2 students: A case study of an ESL science class. In T. Shanahan and F.V. Rodriguez-Brown (Eds.) National Reading Conference Yearbook 49 (pp. 392-404). Chicago, IL: National Reading Conference.

Huyck, E.E., \& Fields, R. (1981). Impact of resettlement on refugee children. International Migration Review, 15, 246-54.

Hynes, T. (2003). The Issue of "Trust" or "Mistrust" in Research with Refugees: Choices, Caveats and Consideration for Researchers. New Issues in Refugee Research, (Working Paper No. 98). Geneva: UNHCR. Retrieved April 22, 2007, from http://www.unhcr.org/research/RESEARCH/3fcb5cee1.pdf Igoa, C. (1995). The Inner World of the Immigrant Child. Mahwah, NJ: Erlbaum. Jensen, L., \& Chitose, Y. (1994). Today's second generation: Evidence from the 1990 U.S. census. International Migration Review, 28, 714-735. 
Jones, C.J., \& Trickett, E.J. (2005). Immigrant adolescents behaving as culture brokers: A study of families from the Former Soviet Union. The Journal of Social Psychology, 145(4), 405-427.

Kaplan, C. (1993). Predicting first-grade achievement from pre-kindergarten WPPSI--R scores. Journal of Psychoeducational Assessment, 11(2), 133138.

Kellaghan, T., Sloane, K., Alvarez, B., \& Bloom, B. (1993). Home environment and school learning. The Jossey-Bass Education Series, (pp. 1-16).

Kia-Keating, M. (2006). Refugee Youth in Resettlement: Exposure to War and Protective Factors. Dissertation Abstracts International: Section B: The Sciences and Engineering.

Kinzie, J.D., Boehnlein, J., \& Sack, W.H. (1998). The effects of massive trauma on Cambodian parents and children. In Y. Danieli (Ed.), International Handbook of Multigenerational Legacies of Trauma (pp. 211-221). New York: Plenum Press.

Kinzie, J.D., Sack, W., Angell, R., Manson, S., Rath, B. (1986). The Psychiatric Effects of Massive Trauma on Cambodian Children. Journal of American Academy of Child Psychiatry, 25 (3), 370-376.

Klesmer, H. (1994). ESL Achievement Project: Development of English as a Second Language Achievement as a Function of Age and Length of Residence in Canada. A special leave project for the Board of Education for the City of North York. 
Kovacev, L., \& Shute, R.H. (2004). Acculturation and social support in relation to psychosocial adjustment of adolescent refugees resettled in Australia. International Journal of Behavioral Development. 28 (3), 259-267.

Kunz, E.F. (1973). The refugee in flight: Kinetic models and forms of displacement. International Migration Review, 7, 125-146.

Lavik, N.J., Hauff, E., Skrondal, A., \& Solberg, O. (1996). Mental disorder among refugees and the impact of persecution and exile: Some findings from an out patient population. British Journal of Psychiatry, 169(6), 726732.

Lee, S. (2002). Learning "America": Hmong American high school students. Education \& Urban Society, 34, 233-246.

Liebkind, K. (1996). Acculturation and Stress: Vietnamese refugees in Finland. Journal of Cross-Cultural Psychology, 27(2), 161-180.

Lustig, S.L., Kia-Keating, M., Knight, W.G., Geltman, P., Ellis, H., Kinzie, J.D., Keane, T., \& Saxe, G.N. (2004). Review of child and adolescent refugee mental health. Journal of the American Academy of Child \& Adolescent Psychiatry, 43(1), 24-36.

Macksoud, M.S., \& Aber, J.L. (1996). The war experiences and psychosocial development of children in Lebanon. Child Development, $67,70-88$.

Martini, M. (1995). Features of home environments associated with children's school success. Early Child Development \& Care, 111, 49-68.

Matute-Bianchi, M. E. (1986). Ethnic identities and patterns of school success and failure among Mexican-descent and Japanese-American students in a 
California high school: An ethnographic analysis. American Journal of Education, 95(1), 233-255.

McBrien, J.L. (2003). A second change for refugee students. Educational Leadership, 61(2), 76-79.

McBrien, J.L., (2005). Educational needs and barriers for refugee students in the United States: A review of the literature. Review of Educational Research, 75(3), 329-364.

McCrone, P., Bhui, K., Craig, T., \& Mohamud, S. (2005). Mental health needs, service use and costs among Somali refugees in the UK. Acta Psychiatrica Scandinavica, 111(5), 351.

McMichael, C. (2002). "Everywhere is Allah's place": Islam and the everyday life of Somali women in Melbourne, Australia. Journal of Refugee Studies, 15 (2), 171-188.

McMurtrie, B., Bollag, B., Brender, A., del Castillo; D., Cheng, M., \& Overland, M.A. (2001). Arab students in U.S. head home, citing growing hostility. Chronicle of Higher Education, 48(6), A42-A45.

Melville, M.B., \& Lykes, M.B. (1992), Guatemalan Indian children and the sociocultural effects government-sponsored terrorism. Social Science and Medicine, 34, 533-548

Miller, A.M., Sorokin, O., Wang, E., Choi, M., Feetham, S., \& Wilbur, J. (2006). Acculturation, social alienation, and depres\$ed mood in midlife women from the former Soviet Union. Research in Nursing and Health, 29(2), 134 146. 
Mollica, R.F., Mclnnes, K., Pham, T., Fawzi, M.C.S., Murphy, E., \& Lin, L. (1998). The dose-effect relationships between torture and psychiatric symptoms in Vietnamese ex-political detainees and a comparison group. Journal of Nervous and Mental Disease, 186(9), 543-553.

Mosselson, J. (2002). Roots and routes: Reimagining the identity constructions of Bosnian adolescent female refugees in the United States. Dissertation Abstracts International, 63(04), 1287. (UMI No. 3048196).

Nguyen, H.H., Messe, L.A., \& Stollak, G.E. (1999). Toward a more complex understanding of acculturation and adjustment. Journal of Cross-Cultural Psychology, 30(1), 5-31.

Nicassio, P.M. (1983). Psychosocial correlates of alienation: A study of a sample of Indochinese refugees. Journal of Cross-Cultural Psychology, 14, 337351.

Normandeau, S., \& Guay, F. (1998). Preschool behavior and first-grade school achievement: The mediational role of cognitive self-control. Journal of Educational Psychology, 50(1), 111-121.

Oetting, E.R., \& Beauvais, F. (1991). Orthogonal cultural identification theory: The cultural identification of minority adolescents. International Journal of the Addictions, 25(5A\&6A), 655-685.

Office of Immigration Statistics (2004). 2003 Yearbook of Immigration Statistics. Washington, DC: Department of Homeland Security. Retrieved April 22, 2007 from 
http://149.101.23.2/graphics/shared/statistics/Yearbook/2003/2003Yearbo ok.pdf

Ogbu, J. U. (1982). Cultural discontinuities and schooling. Anthropology \& Education Quarterly, 13, 290-307.

Ogbu, J. U. (1991). Minority Coping Responses and School Experience. The Journal of Psychohistory, 18(4), 433-456.

Olsen, L. (2000). Learning English and learning America: Immigrants in the eye of a storm. Theory into Practice, 39, 196-202.

Padilla, A.M. (1980). The role of cultural awareness and ethnic loyalty in acculturation. In A. M. Padilla (Ed.), Acculturation: Theory, Models, and Some New Findings (pp. 47-84). Boulder, CO: Westview.

Pagano, R. R. (1998). Correlation. In Understanding Statistics in the Behavioral Sciences, (5th ed., pp. 99-126). Pacific Grove, CA: Brookes/Cole Publishing Co.

Papadopoulos, R. K. (2001). Refugee families: Issues of systemic supervision. Journal of Family Therapy, 23(4), 405-422.

Perez, L. (2001). Growing up in Cuban Miami: Immigration, the enclave, and new generations. In R.G. Rumbaut \& A. Portes (Eds.), Ethnicities: Children of Immigrants in America (pp. 91-125). Berkeley: University of California Press.

Persky, I., \& Birman, D. (2005). Ethnic identity in acculturation research: A study of multiple identities of Jewish Refugees from the Former Soviet Union. Journal of Cross-Cultural Psychology, 36(5), 557-572. 
Phinney, J. S. (1989). Stages of ethnic identity development in minority group adolescents. Journal of Early Adolescence, 9(1-2), 34-49.

Phinney, J.S. (1990). Ethnic identity in adolescents and adults: Review of research. Psychological Bulletin, 108, 499-514.

Phinney, J. S. (1996). Understanding ethnic diversity: The role of ethnic identity. American Behavioral Scientist, 40(2), 143-152.

Pianta, R.C., \& Harbers, K.L. (1996). Observing mother and child behavior in a problem-solving situation at school entry: Relations with academic achievement. Journal of School Psychology, 34, 307-322.

Portes, A. (1999). "Social capital: Its origins and application in modern sociology." Annual Review of Sociology, 24, 1-24.

Portes, A., \& Rumbaut, R. G. (1990). A Portrait. University California Press.

Portes, A., \& Rumbaut, R.G. (1996). Immigrant America: A Portrait ( $2^{\text {nd }}$ ed.). Berkeley: University of California Press.

Portes, A., \& Rumbaut, R.G. (2001). Legacies: The Story of the Immigrant Second Generation. Berkeley: University of California Press.

Portes, P.R., \& Zady, M.F. (1996). Socio-psychological Factors in the Academic Achievement of Children of Immigrants: Examining a Cultural History Puzzle. Office of Educational Research and Improvement: U.S. Department of Education.

Portes, A., \& Zhou, M. (1993). The new second generation: Segmented assimilation and its variants. Annals of the American Academy of Political and Social Science, 530, 74-96. 
Pryor, C.B. (2001). New immigrants and refugees in American schools: Multiple voices. Childhood Education, 77(5), 275-283.

Pynoos, R.S., Kinzie, J.D., \& Gordon, M. (2001). Children, adolescents and families exposed to torture and related trauma. In, E. Gerrity, T.M. Keanne, \& F. Tuma (Eds.), The Mental Health Consequences of Torture (pp. 211-225). New York: Kluwer Academic/ Plenum Publishers.

Reio, T. G., Jr., \& Lasky, S. L. (2007). Teacher risk taking changes in the context of school reform: A sociocultural and cognitive motivational perspective. In D. M. McInerney \& S. Van Etton (Eds.), Standards in Education: Vol. 7. Research on Socio-cultural Influences on Motivation and Learning. Charlotte, NC: Information Age Publishing.

Reynolds, A.J., \& Bezrucko, N. (1993). School adjustment of children at risk through fourth grade. Merrill-Palmer Quarterly, 39, 457-480.

Rice, M., \& Broome, M.E. (2004). Incentives for children in research. Journal of Nursing Scholarship, 36(2), 167-72.

Rong, X.L., \& Preissle, J. (1998). Educating Immigrant Students: What We Need to Know to Meet the Challenges. Thousand Oaks, CA: Corwin Press.

Rousseau, C., Said, T., Gagne, M.J., \& Bibeau, G. (1998). Resilience in unaccompanied minors from the north of Somalia. Psychoanalytic Review, 85(4), 615-637.

Rudmin, F. W. (2003). Critical history of acculturation psychology of assimilation, separation, integration, and marginalization, Review of General Psychology, 7, 3-37. 
Rumbaut, R. G. (1994). The crucible within: Ethnic identity, self-esteem, and segmented assimilation among children of immigrants. International Migration Review, 28, 748-794.

Rumbaut, R.G., \& Portes, A. (2001). The forging of a new America: Lessons for theory and policy. In R.G. Rumbaut \& A. Portes (Eds.), Ethnicities: Children of Immigrants in America (pp. 30-317). Berkeley: University of California Press.

Rumberger, R.W., \& Larson, K.A. (1998). Toward explaining differences in educational achievement among Mexican American language-minority students. Sociology of Education, 71, 69-93.

Sack, W.H., Him, C., \& Dickason, D., (1999). Twelve-year follow up study of Khmer youths who suffered massive war trauma as children. Journal of the American Academy of Child and Adolescent Psychiatry, 38(9), 11731179.

Sam, D. L., \& Berry, J.W. (1995). Acculturative stress among young immigrants in Norway. Scandinavian Journal of Psychology, 36(1), 10-24.

Sarason, S.B., (1972). The Creation of Settings and the Future Societies: (1972 reprinted 1988). San Francisco: Jossey-Bass.

Shanahan, M. J., Mortimer, J. T., \& Kruger, H. (2002). Adolescence and adult work in the twenty-first century. Journal of Research on Adolescence, 12, 99-121. 
Sinclair, M. (2001). Education in emergencies. In J. Crisp, C. Talbot, \& D.B. Cipollone (Eds.), Learning for a Future: Refugee Education in Developing Countries ( $\mathrm{pp}$ 1-84). Lausanne, Switzerland: United nations Publications. Sokoloff, B., Carlin, J., \& Pham, H. (1984). Five-year follow-up of Vietnamese refugee children in the United States. Clinical Pediatrics, 23, 565-570.

Sourander, A. (1998). Behavior problems and traumatic events of unaccompanied refugee minors. Child Abuse \& Neglect, 22(7), 719-727.

Stein, B. N. (1979). Occupational adjustment of refugees: The Vietnamese in the United States. International Migration Review, 13, 25-45.

Stein, B. N. (1980). Refugee Resettlement Programs and Techniques: A Research Report Submitted to the Select Committee on Immigration and Refugee Policy. East Lansing, MI: Michigan State University Resource Center for Refugee Resettlement.

Stein, B.N. (1981). The refugee experience: Defining the parameters of a field of study. International Migration Review, 15, 320-330.

Stipek, D.J., \& Ryan, R.H. (1997). Economically disadvantaged preschoolers: Ready to learn but further to go. Developmental Psychology, 33(4), 711 723.

Stutters, A., \& Ligon, J. (2001). Differences in refugee anxiety and depression: comparing Vietnamese, Somalian, and Former Yugoslavian clients. Journal of Ethnic and Cultural Diversity in Social Work, 10(1), 85-95.

Suarez-Orozco, C., \& Suarez-Orozco, M.M. (2001). Children of Immigration. Cambridge, MA: Harvard University Press. 
Summerfield, D. (1999). A critique of seven assumptions behind psychological trauma programs in war-affected areas. Social Sciences and Medicine, $48,1449-1462$.

Sung, B. L. (1987). The Adjustment Experience of Chinese Immigrant Children in New York City. New York: Center for Migration Studies.

Swindle, R.W., \& Moos, R. H. (1992). Life domains in stressors, coping, and adjustment. In W.B. Walsh, K.H. Craik, \& R.H. Prices (Eds.), Person Environment Psychology. Hillsdale, NJ: Earlbaum.

Thomas, W.P., \& Collier, V. (1997). School Effectiveness for Language Minority Students. (NCBE Resource Collection Series No. 9). Washington, DC: National Clearinghouse for Bilingual Education. Retrieved July 8, 2008 from www.ncela.gwu.edu/ncbepubs/resource/effectiveness/

Tollefson, J. (1989). Alien Winds: The Reeducation of America's Indo-chinese Refugees. New York: Praeger.

Tousignant, M., Habimana, E., Biron, C., Malo, C., Sidoli-LeBlanc, E., \& Bendris, N. (1999), The Quebec adolescent refugee project: Psychopathology and family variables in a sample from 35 nations. Journal of the American Academy of Child and Adolescent Psychiatry, 38, 1426 - 1432

Tremblay, R.E., Masse, B., Perron, D., LeBlanc, M., Schwartzman, A.E., \& Ledingham, J.E. (1992). Early disruptive behavior, poor school achievement, delinquent behavior, and delinquent personality: Longitudinal analyses. Journal of Consulting \& Clinical Psychology, 60, 64-72. 
Trickett, E.J., \& Birman, D. (2005). Acculturation, school context, and school outcomes: Adaptation of refugee adolescents from the Former Soviet Union. Psychology in the Schools, 42(1), 27-38.

Trueba, H.T., Jacobs, L., \& Kirton, E. (1990). Cultural Conflict and Adaptation: The Case of Hmong Children in American Society. New York: Falmer Press.

U.S. Committee for Refugees and Immigrants (2004). World Refugee Survey 2004. Retrieved April 22, 2007 from http://www.refugees.org/article. $\operatorname{aspx?id=1163}$

U.S.Bureau of the Census (1990). Internet release date June 15, 1998.

United Nations High Commissioner for Refugees (1994). Refugee Children:

Guidelines and Protection. Retrieved July 5, 2008 from the website of the United Nations High Commissioner for Refugees, http://www.unhcr.ch/cgi bin/texis/vtx/protect/opendoc. pdf?tbl=PROTECTION\&id=3b84c6c67

United Nations High Commissioner for Refugees (1996). Convention and Protocol: Relating to the Status of Refugees. Retrieved April 22, 2007 from http://www.unhcr.org/protect/PROTECTION/3b66c2aa10.pdf United Nations High Commissioner for Refugees (2000). The UNHCR Global Report 1999. New York: Oxford University Press.

United Nations High Commissioner for Refugees (2002a). Refugees by Numbers 2002. Retrieved April 24, 2007 from http://www.unhcr.org.ch 
United Nations High Commissioner for Refugees (2002b). Special Feature on the $50^{\text {th }}$ Anniversary of the Convention. Retrieved April 24, 2007 from http://www.unhcr.org/1951 convention/index.html.

United Nations High Commissioner for Refugees (2005). 2004 Global Refugee Trends: Overview of Refugee Populations, New Arrivals, Durable Solutions, Asylum Seekers, Stateless \& Other Persons of Concern to UNHCR. Geneva: UNHCR Population and Geographical Data Section Division of Operational Support.

Vinokurov, A.Y., Trickett, E.J., \& Birman, D. (2002). Acculturative hassles and immigrant adolescents: A life-domain assessment for Soviet Jewish refugees. The Journal of Social Psychology, 142(4), 425-445.

Watt, D.L.E. \& Roessingh, H. (1994). Some you win, most you lose: Tracking ESL students' drop out in high school (1988-1993). English Quarterly, 26(3), 5-7.

Weine, S.M., Vojvoda, D., Becker, D.F., McGlashan, T.H., Hodzic,E., Laub, D., Hyman, L., Sawyer, M., \& Lazrove, S. (1998). PTSD symptoms in Bosnian refugees 1 year after resettlement in the United States. American Journal of Psychiatry, 155(4), 562-564.

West, J., Denton, K., \& Germino-Hausken, E. (2000). America's Kindergartners: Early Childhood Longitudinal Study - Kindergarten Class of 1998-99, Fall 1998 [Statistical analysis report]. Washington, DC: U.S. Department of Education, National Center for Education Statistics. Retrieved July 6, 2008 from http://nces.ed.gov/pubsearch/pubsinfo. asp?pubid=2000070 
Westermeyer, J., \& Wahmanholm, K. (1996). Refugee children. In R.J. Apfel \& B. Simon (Eds.), Minefields in their Hearts: The Mental Health of Children in War and Communal Violence (pp. 75-103). New haven: Yale University Press.

White, M. and Kaufman, G. 1997. Language usage, social capital, and school completion among immigrants and native-born ethnic groups. Social Science Quarterly, 78 (2), 385-398.

Whittaker, S., Hardy, G., Lewis, K., \& Buchan, L. (2005). An exploration of psychological well-being with young Somali refugee and asylum-seeker women. Clinical and Child Psychology and Psychiatry, 10, 177-196.

Wilkinson, L. (2002). Factors influencing the academic success of refugee youth in Canada. Journal of Youth Studies, 5(2), 173-193.

Willms, J.D., \& Jaconsen, S. (1990). Growth in mathematics skills during the intermediate years: Sex differences and school effects. International Journal of Educational Research, 14, 157-174.

Wingfield, M., \& Karaman, B. (2001). Arab Stereotypes and American

Educators. Retrieved July 5, 2008 from the website of the American-Arab Antidiscrimination Committee, http://www.adc.org/index.php?id=246

Wingfield, M., \& Karaman, B. (2002). Arab stereotypes and American educators. In E. Lee, D. Menkart, \& M. Okazawa-Rey (Eds.), Beyond Heroes and Holidays: A Practical Guide to K-12 Anti-Racist, Multicultural Education and Staff Development (pp.132-136). Washington, DC: Teaching for Change. 
Wood, P. B., \& Clay, W. C. (1996). Perceived structural barriers and academic performance among American Indian high school students. Youth and Society, 28 (1), 40-61.

Zarowsky, C. (2000). Trauma stories: Violence, emotion and politics in Somali Ethiopia. Transcultural Psychiatry, 37, 383-402.

Zarowsky, C. (2004). Writing trauma: emotion, ethnography, and the politics of suffering among Somali returnees in Ethiopia. Culture, Medicine and Psychiatry, 28(2), 189-209.

Zea, M.C., Asner-Sel f, K.K., Birman, D., \& Buki, L.P. (2003). The Abbreviated Multidimensional Acculturation Scale: Empirical validation with two Latino/Latina samples. Cultural Diversity \& Ethnic Minority Psychology, 9(2), 107-126.

Zhou, M. (2001). Straddling different worlds: The acculturation of Vietnamese refugee children. In R.G. Rumbaut \& A. Portes (Eds.), Ethnicities: Children of Immigrants in America (pp. 187-227). Berkeley: University of California Press.

Zhou, M., \& Bankston III, C.L. (2000). The biculturation of the Vietnamese student. ERIC Clearinghouse on Urban Education, 152, 1-7. 


\section{LIST OF APPENDICES}

\section{APPENDIX \\ PAGE}

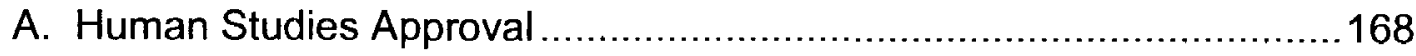

B. Language Identity Behavioral Acculturation Scale (LIB) ...................172

C. Strengths and Difficulties Questionnaire (SDQ).............................176 
APPENDIX A - HUMAN STUDIES APPROVAL 

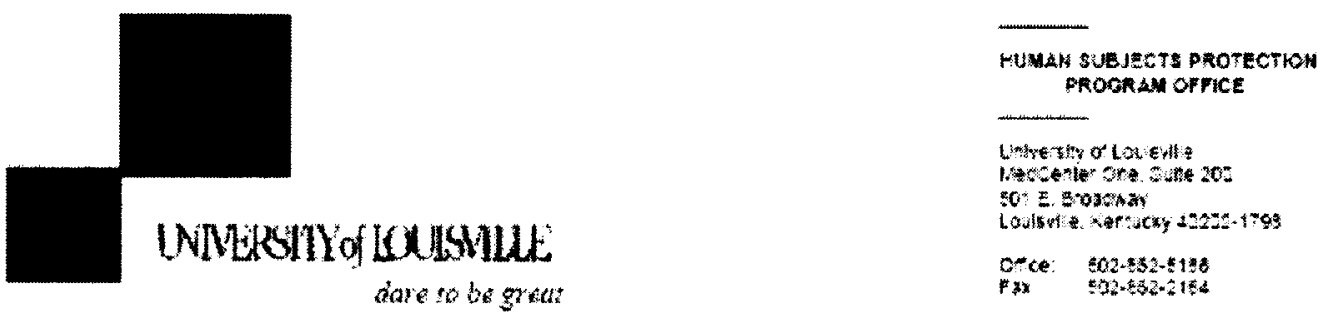

B.y 77,2007

Thomas Reio. Ph D.

(Mandesena Sewhon)

Leadershio Foundatione 8

Human Respurce Ecucation

CEHD-Umigersty of Lowisule

Lousvile $K Y 40282$

RE: 249.07 Accuturaton \& School Adjusment of Somali Bantu Refuge Chidren

Ciear Dr Peio

The revsed informed consents 21 , apolication and questionnate claritiotions for the above referenced study nave heen received and contain the changes requested in cur letter of $5: 1607$

This study has been reviewed by the chair of the Institutional Revew Board IRB anc approved throug the Expedteo Review Procedure. accotong to $45 \mathrm{CF} 40.1106 \mathrm{~b}$ ) siche the research is on indivdual or grow charactertics or benaviors

The folowng tems have heen aporoved

- Protocol and Ouestionares, not dated

- Revised inomed Consen (subect) daed 5:1607

- Reviser intomed Consent (Teacher, dated 51707

- Suhect Assent dated 50107

This study how has final IRB approval through 5:16:2008 The committee will be advised of this action at ther next full Board meeting

Please note that the IRB follows the principes of the Belmon: Report is in conplance with Good Cincal Practice Gudelines as defined by the 1.3 . Food and Drug Administration and the Department of Health and ruman Services under the Code of Federal Regulations (2) CFR Pars 50 and 56,45 $C F R 46$ and Intematonal Conterence on Hamonization ilctil Guidelnes (Section Es:

This study was approved for children under category 1. 4E CFR 45.404 - Research not involving greater than minimal rish. No greater than minimat risk to chileren is presented. ony if the IRB finds that adequate provisons are thate for solititing the assent of the children and the dermission of their parents or guardians. as set forth in 3 et. 46.408 . This category requires the assent of chid if over age 7 and at last one parent signature. 
Site Approval

If this study will take pace ousde of the Jiversity of Louswile camouses. site aporoval from the organcation must he given before the research may begin

You should complete and return the Progress Reporticontinuation Request Form EIGHT weeks pior to 5.162008 , in order to ensure that no lapse in approval occurs. Best wishes tor a successfus siody.

Please send al mquires and electonic revisedirecuested tems to our ofice email aodress at nsopotericuizulle edu.

$\operatorname{sinceres}$

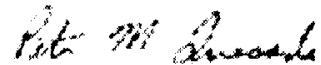

Peter M cusada Fn D ve Char

SocaliEenaworaliedutional Institutional Review Board

PAldor: 


\section{vetferson county 19 Shaping the Future}

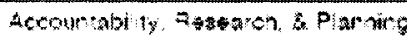
339. Newhur Fon

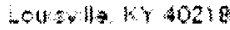

\begin{tabular}{|c|c|}
\hline phors & 15021485.3030 \\
\hline & 14921485.6255 \\
\hline E.thot & 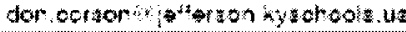 \\
\hline
\end{tabular}

May 1\%. 2007

Wanbers sisknon

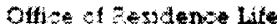

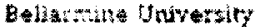

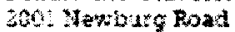

Lo wis

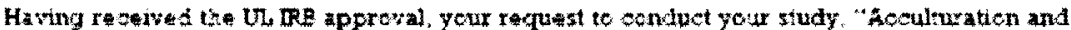

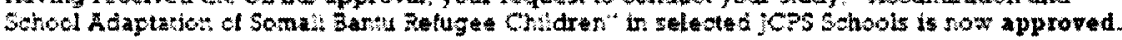

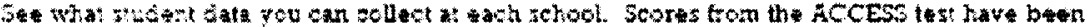

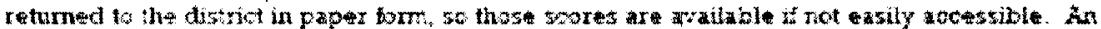

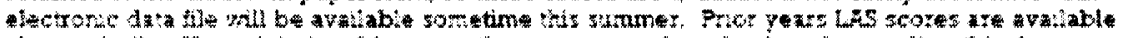

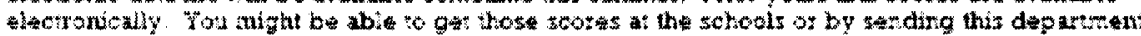

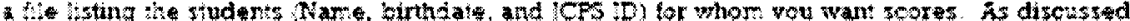
or the phose. flementary students do no have grade point averages. Tete some disaplinary inforration arablable tom this ottioe

Sitcettity

\section{Danald L. Corsun}

Donald Corson

internal Revew Board (IFB)

VanHoose Elucationa Cente

3332 Newburg Road

Lovisulle, KY 40218 
APPENDIX B - Language Identity Behavioral Acculturation Scale (LIB) 
The "Language, Identity, and Behavior" (LIB) Acculturation Measure (Adapted from Birman \& Trickett, 2001)

\section{A. Language}

For the following statements, please mark one of the three possible answers.

How would you rate your ability to speak English:

1. overall?

2. at school

3. on the phone

4. with American friends

5. with strangers

How well do you understand English:

6. overall

7. on TV or in movies

8 . books in school or in magazines

9 . in songs

How would you rate your ability to speak Mai Mai:

10. overall

11. with family

12. with Somali friends.

13. on the phone

14. with strangers

How well do you understand Mai Mai:

15. overall

16. on TV or in movies

17. in songs
A lot Sort of No

23

23

23

23

23

23

23

23

23

23

23

23

$2 \quad 3$


The "Language, Identity, and Behavior" (LIB) Acculturation Measure (Adapted from Birman \& Trickett, 2001)

B. Cultural Identity:

How would you describe your cultural ethnic identity:

To what extent are the following statements true for you?

A lot Sort of No

1. I think of myself as being American .

$2 \quad 3$

2. I feel good about being American ............................................. 1122

3. I strongly feel I am American................................................... I 203

4. I am proud of being American..................................................... 112

5. I think of myself as being Somali ...............................................1 2

6. I feel good about being Somali .................................................... 112

7. I strongly feel I am Somali ........................................................1 1

8. I am proud that I am Somali .................................................... 
The "Language, Identity, and Behavior" (LIB) Acculturation Measure (Adapted from Birman \& Trickett, 2001)

C. Cultural Participation ("Behavioral Acculturation")

To what extent are the following statements true about the things that you do?

How much do you speak English:

A lot Sort of No

1. at home?

2. at school?

23

3. with friends (Americans)?.

23

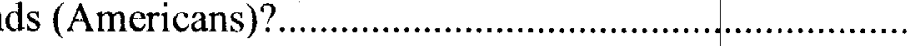

23

How much do you:

4. read American books or magazines? .......................................... 1

5. listen to American songs? ..........................................................1 112

6. watch American movies (on TV, VCR, etc?) ................................ 122

7. eat American food? .............................................................. 112

8. have American friends? ......................................................... 1122

9. attend parties at American kids' homes? ..................................... 122

How much do you speak Mai Mai:

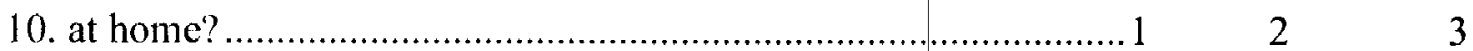

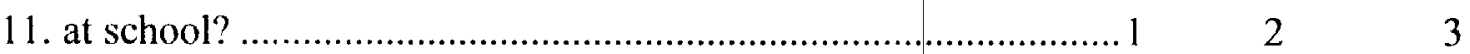

12. with Somali friends? ............................................................. 1

How much do you:

13. read Somali books or magazines?............................................. 1

14. listen to Somali songs? ........................................................ 1122

15. watch Somali movies (on TV, VCR, etc?) ............................. 1122

16. eat Somali food? .................................................................... 1

17. have Somali friends?............................................................ 1122

18. attend parties at Somali kids' homes? .....................................1 2 
APPENDIX C - STRENGTHS AND DIFFICULTIES QUESTIONAIRE (SDQ) 


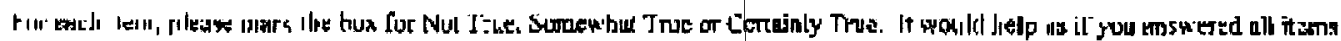

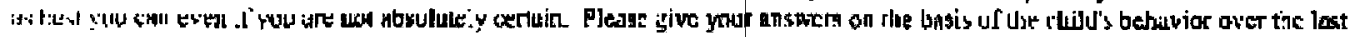

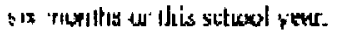

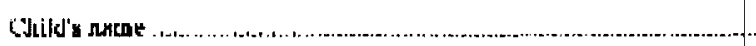

Hale'Famalc

Dith ol birth

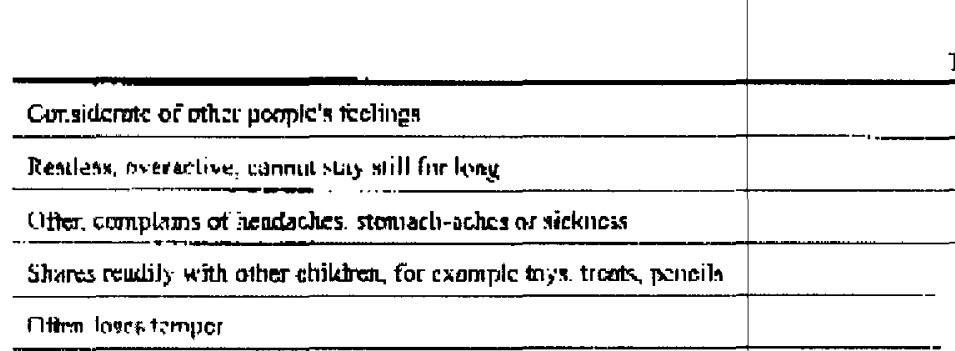

Nol sumenbst Cistuinjy Trule True True

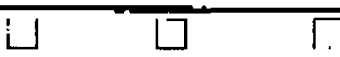

$\square \square$

$\square-\square \quad \square$

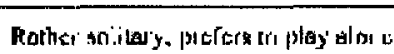

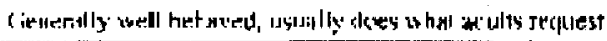

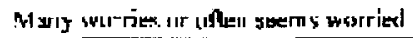

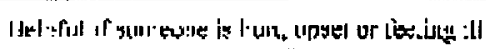

Couscautly fidgeriug ne st)ull'ring

\begin{tabular}{lll}
1 & $\square$ & $\square$ \\
\hline$\square$ & $\square$ & $\square$
\end{tabular}

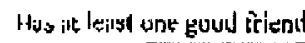

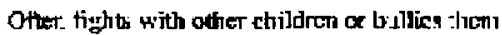

Otter, anhappy, depicased pl' teazil

Gcielelly liked by other dildetin

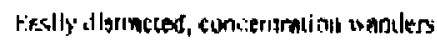

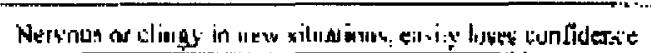

Kind wa joungers childres

Ones! lies or cuentls

Piaked on or tullied by othet childres

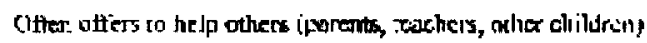

\begin{tabular}{|l|l}
\hline Thinky shimisy out betouc acing & \\
\hline
\end{tabular}

\begin{tabular}{l|l}
\hline Stably trota hame, gchool of elsezihere & \\
\hline
\end{tabular}

Gets along bater with adulk then will olter thild teat

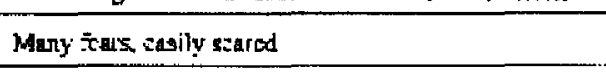

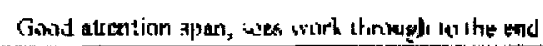

$\square \longdiv { \square } \square$

Signature

$\square \quad \square \quad \square$

\begin{tabular}{lll}
$\square$ & $\square$ & $\square$ \\
\hline & $\square$ & $\square$
\end{tabular}

Panent : Tealliher / Oth a (Plense specifyci 


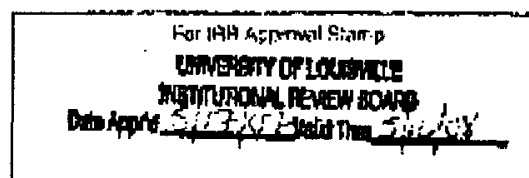

\section{Subjogt Informed Consant Document}

Acculturation and School Adaptation of Sornali Bantu Pafugae Childran

Investígatorig) name \& adoress:

Thomas Reio, Ph.E.

Leadership, Foundations, afd Hurnen Resource Education

College of Education ard Human Devolopment

Un:verşity of Louisville

Louiswillo, KY 40292

Maubeena Sekhon, M.A.

Education \& Counseling Psychology, RM 320

Collega of Education and Human Davelopment;

University of Lotriswilla

Lovisuilla, KY 40292

Siteis) where study is to be conducted:

Layno Elomantary School

Wilkerson Eiementary School

Gamp Taylor Elementary School

Brandeis Elemrentary School

Storiestrea: Elamentary School

Phone number or subjects to call for ouestions:

(502) 852-0639

Introduction and Batakground Information

Yout chlld is invited to partclpate In a research study. The study is being conducted by Thomas Reio. Ph.D.. and Mankeena Sekhory. M.A. The study is sponsored the University of Louiswille, Depa tment of Education and Courseling Psychology. The study will teke place at Layne Elementary Schnod. Wilkerson Elamentary Schonl, Gamp Tuylor Elomantury Sciscol, Brarndeis Elomonrary School and Stonostracr Elementary Sehooi. Approxirnately 120 subjec!s vill be invited to participate.

\section{Purpose}

Tre puppose of this sturiv is ro better undersiatd the needs of Somali Bantu rofugee child fon by examinimg their adjustment to the Unied States as well as te their schools in th.o Uinted States. 


\section{Mocurturotion, Adjustment \& Bchacl Oulcomes of Samali Eantu Fefugea Children \\ Proceduree}

In this study, wour child will be asked to complete the "Language, Identity, and Behavior" Scals to help us inderstand how well you thirk you speak and understand English and Mai-Mai, hisiher culturab identity and participalion in American and Somalj Bantu cultural activities. A Somali Bantu translator wili orally administer the questions and your child will respond by circling the approprate responso (1- 2 lot, 2 - sart of, 3 - no). This should take you no longer than 30 minutes to complete. Your ehild has the right to dec:ine your rasponge to fny quastions that Trily maks hirn/her uncomforiable

\section{Potentlal Rloks}

Thire are no loresesable risks, although there may be unforeseen risks

\section{Beneflts}

The possible benelits of this study include teschers, counselors, and administrators, gaining an increased undarstanding and knowledge of the Somall Bantu refugee children's needs. adjustment to the United Stetes, and their school adaptation in the United States. The information collected may not benefif your child directly. The iniomaton leamed in this study may be helptul to othors.

\section{Confdentiality}

Tolal privacy canfal be guaranteed. Your child's privacy will be protecled to the extert permitted by law. If the results from this study are publishod: your childs name will not be made public. While unlikely, the following may look at the study rocords:

The University of Louisville Institutional Review Board. Human Subjects Protection Program Office

Ottice for Human Research Protectio-76 (OHAPy,

In order to ensure that the data collated is secuied, it will be placed in a locked filing cabinet in a secure area within the College of Educahion and Hur an Devalepment at the Univarsity of Loussvilie.

\section{Voluntary Participation}

Taking part in this stady is volunkary. Yout child may chooso not to take part at oll. If your child decides to be in this study heishe may stop taking part at ary tilme.

\section{Hesearch Subject's Aights, Questons, Concerns, and Complainte}

If you have any ocncerns nr complaints ahout the study of the study staff, ycu heve hroe optinns.

You may conlacl the pincipet investigator at $\{502), 952-0639$

If you have any questions about vour pights as a study subject, questions, cencerr!s or complints. yois may calt the Human Sibjects Proiection Program OHice (HSPPG) !502; 85e-51a8. You may disciss any guestions aboul youtur rigints as a subject. in secrei. whitr a

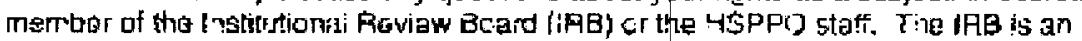

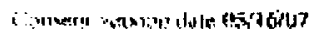

Thipla : 


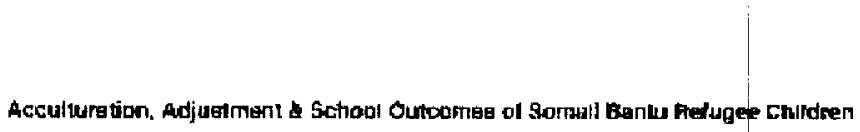

isdopendant committes composed of mombers of the University comimunity, staff of the institutions, as well as lay members of the community not connected with these institutions. The HAB his revicwed this study

If you want to speak to a person outside the thiventity, you nay call $1-877-862-1167$. Yow wili be given the chance lo lalk about any questions concems or complaints in socrot. This is a 24 fucur hor line answered by people who do wot wotk at the Universily of Locisville.

Thas paper teils your child what will happen during the study if he'she chooses to take pan. Your signat:Je rmar:s that this study has ban discissen with you, that your questions have been answered, and that your child will take part in the study. This intprmed consent docurnent is not a contract. You aue nat giving up any legel nghts by eigning this informed consent document. You will be given a signed copy of this paper to heep lar your fecords.

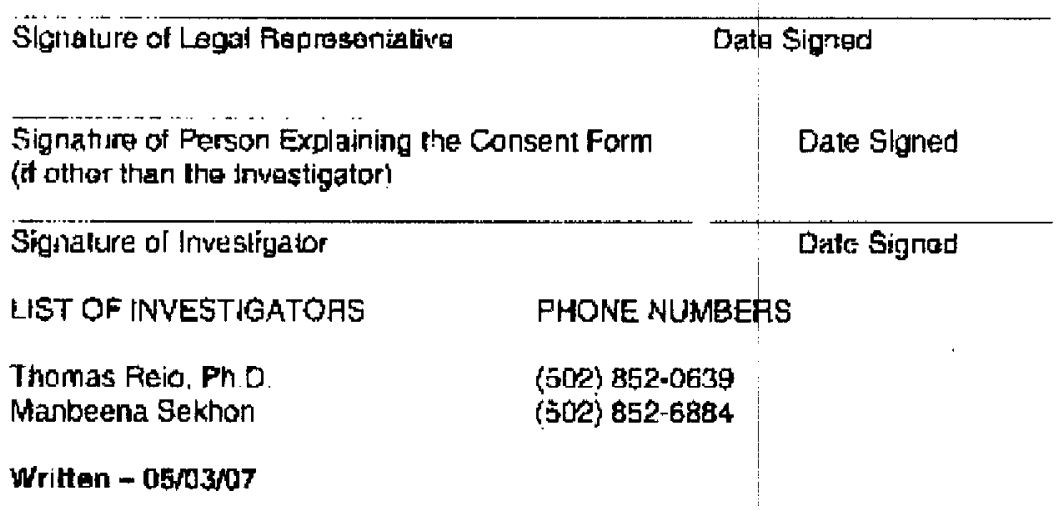

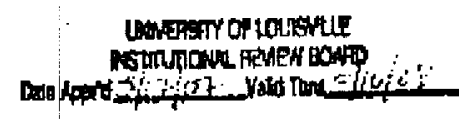

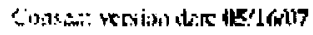

Puge 3 , ग? 


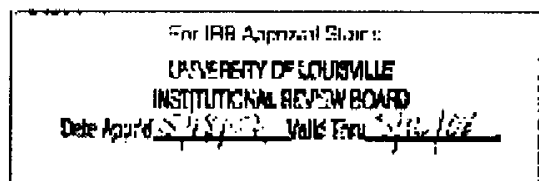

\section{Subjact (Toachar) Informed Cohsent Document}

\section{Acculturation and School Adaptation of Somali Bantu Refugee Chlldren}

Investigator! s) name $\mathbf{8}$ address:

Thomas Reia. Ph.D.

Leadarship, Foundations, and Human Rosourco Education

College of Education and Human Development

University of Loulsuille

Lourisville, KY 40292

Markbena Sekhon, M.A

Ed:scation \& Counseling Psychology: RM 320

College of Educution and Humas Developmentt

University of Louiswille

Louisville, KY 40292

Siters) where study is to be concucted

Layne Elementery School

Wilkerson Elemenlary School

Camp Taylor Elementary Schnol

Buandeis Elementary School

Stonestreel Elementary School

Phone number for subjects to call for quesions:

$(502) 852-0639$

\section{Introduction and Background information}

You are invited to pasticipate in es iesearch study. The study is baing conducted by Thomas Reid, Ph.D., and Manbegna Sekhon. M.A. The study is sponsoled the Univgrsity af Louisvilie: Depsrtmignt of Edication and Counseling Paychology. The study will take pluce at Layne

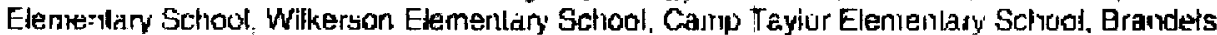
Elemeatary School and Stonestreet Elementaly School.

Approximalely 120 children and the 5 Esh teachers form each of the 5 identhed ceiemeniary schools nifl be invited to participato.

Purpose

Tho frilpose of this study is to better Jiderstand the reeds of Somali Bentis refugee children by examining their adjustitient to she untted Stales as well as :o their sohools in the Unled Sates.

lityed 1 in 


\section{Procedures}

In this study. you will be asked to complete the "Strengths and Difficulves Questionnaire." This doctment is a brief behaviogral screonirg queslionnalre on each of your Somali Rantu students. It should take you about 3.5 mirutes lo complete thls insirument for each studend.

\section{Potential Aisks}

There afe no foreseeable risks, athough thera may bo unforbseen risks.

\section{Benefits}

Tha possibie benefits of this sludv inctude teachers, counselors, and. administrators. gaining an incregsed understanding and inowledge of the Somali Bantu rofugce children's necds, adjustment lo the Uniled Slales, and their school adaptation in the United States. The information collected may not benefit you difectly. The information asarsed in this study may be relpful to others.

\section{Comfidentiality}

Total privacy cennol be guaranteed. Youl privacy will be protected to the extent permitled by law. If the results from this study are published: your name will not be mede public. While unlikely, the following Tay look at the study records:

The University of Louisville Institution Iel Review Board. Human Subjects Protection Program Oflice

Olfice lor Human Research PIotections (OHPP),

in order to ensure shat the data colleted .s secured, it will be pleced in a locked fling cabinet in a secure area within tho Colleggc of Education and Human Development at the University of Lousgrillo.

\section{Voluntary Participation}

Taking part in this study is voluniary. You may choose nol lo lake parl at all. If you decide to bo in this study you may stop iaking part at ary lime.

\section{Retearch Subject's Rlght, Questlons, Concerns, and Complaints}

If you have azy concerins of complaints about the stidy or the study staff. you fave three cptions.

You may contuct the principed investinator at (502) 0.52-06.99

If you have any questions about your rights as a study subject, quaștions. Doncerna or complaints, you may call the Human Subjents Protaction Proguam Oflice (HSPPO) (502) 852-5:88. You may discuss any quastions about your rights as a subject. in secrer, wh tha member of the Institutional Heview Board (IFB) or the HSPPO stalf. The !AB is an

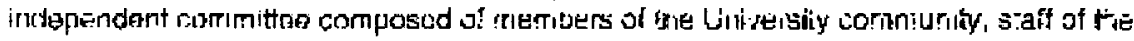


institulions. as well as lay members of the community not connected with these institutions. The IPB has reviewed this study.

If you want to speak io a persor outgide the Univensity, you mry call 1-B77-852-1167. You

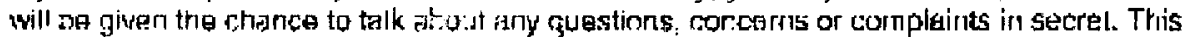
is a 24 hour hat ling answered by people who de not work the Unwersity of LaLisville.

This paper tells you what wlll happen during the study if you choose to take part. Your signazuye meens that this study has bean discussed with ycu, that your questions have boen answored, and that you will take past in the study. This istomed consent cocument is not a cortracl. You nfe not giving up ary legal rights by signing this informed consent document. You wili be given signed copy of thrs piper to koep for your reseords.

\begin{tabular}{|c|c|c|}
\hline \multicolumn{2}{|c|}{ Signalure of SubjectlLegal Representative } & Date Signac \\
\hline \multicolumn{2}{|c|}{$\begin{array}{l}\text { Signature of Person Explaining the Consent Form } \\
\text { (if oither thath the Investigator! }\end{array}$} & Dete Signed \\
\hline Signature of Investigator & & Date Signod \\
\hline LIST OF INVESTIGATOAS & \multicolumn{2}{|c|}{ PHONE NUMBEFS } \\
\hline $\begin{array}{l}\text { Thomas Reio. Ph. D. } \\
\text { Manbeena Sekinon }\end{array}$ & \multicolumn{2}{|c|}{$\begin{array}{l}(502) 852-0639 \\
(502) 852 \cdot 6884\end{array}$} \\
\hline \multicolumn{3}{|l|}{ Written - 05/03/07 } \\
\hline
\end{tabular}

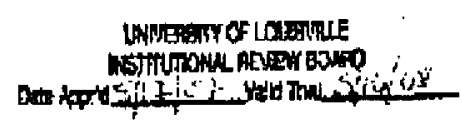

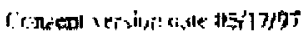

Pugre 3 , : : 


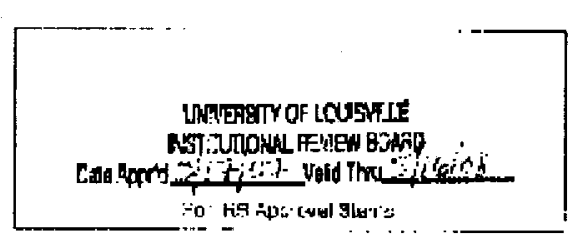

SUBJFCT ASSENT

\title{
ACCUH.TURATION AND SCHOOL ADAPTATION OF SOMALI BANTU REFUGEE CHILDREN
}

\begin{abstract}
I sm invited to be it a reseasch study being done by Professor Thon'as Reiv. Whe'l a person is : 7 researsh study, they are called a "subject". I am invited because I am a Somak Bantu retugee stwiying at an elementury schicol in Louisville.

This treecris that I will answer snme questions about my life i!k Aunerica regarding how mac 7 E aglish and Mai-Mai I speak and understand. about who $f$ thirix $f$ arm: as a persor. (Sonali Bantu and / or American) and Somali Bantu and / or American cullural activitjes I participatt? il There may be some rizks with this atludy For example havilsg to alsswer questions inay IJake mise feel unconifortable.
\end{abstract}

Tris study will last about 15-30 nrilutes. The kenefit to rove for patticipating in this study is that it whll help my teachers understand how I am adjusting to life in America and to the school d a.n study:ng in.

My family, the professor, sct:ool principal and my ESL toacher will know that I' $m$ in the study. If anyonio a ise is given information about me, they wil. not know my name. A : ymber or initials will be used instead of my name

have Deen told about th s study and know why it is being done anc what I have bo do. My parenti(i) have agreed to let me be in the siudy. If I have ally questions I can usk Professor Tromas Reio. He wil: aliswer rny questions. If I do not want to be in this study or I warit to quit after I am alseady in this sludy, I can fell tha resoarcher and he will discuss this with my parente.

Printed Name of Gulejec:

Signature of Sitejed Dale Signed

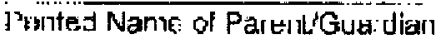

Signatura of Parant/Gitardian Date Signed

Printad ivame of Investigator

Sigrature of Investicator

Date Signed

Astent varion date losigeior 


\section{CURRICULUM VITA}

\section{MANBEENASEKHON}

\section{EDUCATION}

Aug 2003- University of Louisville, Louisville, Kentucky

Present Ph.D. program in Counseling Psychology (APA-Accredited Program)

Dissertation: Acculturation \& School Adjustment of Somali Bantu Refugee

Children

Degree Expected: August 2008

Aug 2000- University of Dayton, Dayton, Ohio

May 2003 M.A. in Clinical Psychology

Thesis: Differences amongst Field Dependent $e^{-\infty}$ Independent Individuals in their Reations to Paintings.

Aug 1996- University of Pune, Pune, India

May $2000 \quad$ B.A. in Psychology

\section{CLINICAL EXPERIENCE}

Aug 2007- Pre-Doctoral Psychology Internship (APA-Accredited)

Present Student Counseling Services

Miami University, Oxford, Ohio

- Provide initial evaluation and referral services

- Provide time-attendant individual and family therapy

- Co-facilitate general process and structured psycho-educational CBT groups (body image and eating disorders, substance abuse, international student support group - cross cultural issues)

- Specialization rotations: women's issues (eating disorders, sexual assault/ abusc), substance abuse, multicultural counseling, international student issues, refugee and immigrant needs, supervision, and teaching.

- Conduct substance abuse assessments, attention problem evaluations (APEs), career, personality and cognitive assessments including clinical intervicws, administering, scoting, interpreting results, and delivering feedback.

- Develop and conduct outreach projects and workshops

- Provide clinical supervision to a doctoral level clinical psychology placement student (paid position, 20 hours per week) 
- Provide on - call crisis intervention services during and after hours

- Scrve on intern selection committec

Supervisors: Pam Wilkins, Ph.D., Licensed Psychologist

Kip Alishio, Ph.1 D., Licensed Psychologist

June 2007July 2007

Jan 2006-

Dec 2006

June 2006July 2006

May 2004-

June 2006

\section{Pre-Doctoral Advanced Practicum/ Human Services Specialist} Seven Counties Services (CMHA), Louisville, KY

- Worked with multi-disciplinary staff in various formats to assist with the summer program including conducting initial assessments as well as group therapy for children and parents with a wide range of psychiatric, mental health and behavioral problems

Supervisor: Elizabeth Jackson, Ph.D., Licensed Psychologist

\section{Pre-Doctoral Practicum/ Psychological Assessment}

Lifespring Mental Health Services/ Dual Diagnosis (CMHA), Jeffersonville, IN

- Administer, score, and report intelligence and personality assessments

- Write integrated psychological reports

Site Supervisor: Heather Henderson-Gallighan, Ph.D., Licensed Psychologist University Supervisor: Patrick Hardesty, Ph.D., Licensed Psychologist

\section{Senior Therapeutic Aide, Kentucky Impact}

Seven Counties Services (CMHA), Louisville, KY

- Provided in-home services to children with severe emotional and behavioral problems

Supervisor: Sharon Sanders, ICCSW

\section{Pre-Doctoral Advanced Practicum/ Human services}

Seven Counties Services (CMHA), Louisville, KY

- Provided individual, family and group psychotherapy and psychotherapy and psycho-education to a diverse population of children, adolescents, and adults with a wide range of psychiatric, mental health and behavioral problems

- Conduct initial evaluations, needs assessments, develop treatment plans and provide appropriate referrals to various institutional programs and services

- Consultation to staff conducting the Families in Transition Program for parents undergoing divorce and mandated by Jefferson County Court System

- Coordinate with caregivers, schools, courts, therapists and other service providers to create and monitor treatment plans aimed at reducing negative symptoms

- Participate in weekly collaborative treatment planning

Site Supervisor: Elizabeth Jackson, Ph.D., Licensed Psychologist

University Supcrvisors: Kathleen Kirby, Ed.D., Licensed Psychologist, Patrick Hardesty, Ph.D. Licensed Psychologist 
May 2005

\section{Psychology Traince}

Families in Transition Program, Jefferson County Courts, Louisville, KY

- Completed extensive training and co-led court mandated psycheducational program for families with parents undergoing divorce.

Aug 2002

\section{Residential Treatment Specialist}

Aug 2003

St Joseph Children's 'Treatment Center, Dayton, Ohio

- Provided direct cate and group management of severely emotionally/ mentally disturbed youth in both open and intensive locked residential facilities

- Implemented treatment directed activities and program routines

- Conducted extensive documentation of behaviors

- Implemented individual behavior modification plans

- Organized and led recreational and therapeutic activities for the youth

- Provided crisis intervention, including physical restraints and incident debricfing

Sep 2001- Psychology Intern

Aug 2002 Catholic Social Services, Dayton, Ohio

- Co-led individual, couple and family therapy sessions

- Conducted mental status examinations

- Participated in weekly treatment planning

- Observed intensive treatment program for children with Reactive Attachment Disorder

Jan 2001- Psychology Intern

Aug 2001 Grandview Hospital (Inpatient \& Outpatient Psychiatric Units), Dayton, Ohio

- Co-led client initial evaluations, family therapy, \& chemical dependency groups

- Facilitated therapeutic groups utilizing art and recreational therapy techniques

- Provided crisis intervention and stabilization

- Participated in weekly treatment planning

\section{RELATED WORK EXPERIENCE}

Aug 2006-

July 2007

\section{Graduate Residence Hall Director}

Bellarmine University, Louisville, Kentucky

- Duties involved administrative tasks, advising/ programming, counseling, mediation/ policy enforcement with first y car residents

- Supervision of six resident assistants

Group Leader, 2005 Youth Forum for Peace Building Peacemaking Skills in Youth, Louisville, Kentucky

- Middle school group leader 
Feb 2004-

Student Mentor

May 2004 Noe Middle School, Louisville, Kentucky

- Provided in-class mentoring and tutoring to at risk middle school students

Aug 2000- Student Assistant, Office of Enrollment Management

April 2003 University of Dayton, Dayton, Ohio

Aug 2000- $\quad$ Student Assistant, Current Periodicals, Roesch Library

April 2003 University of Dayton, Dayton, Ohio

\section{RESEARCH EXPERIENCE}

July 2003-

\section{Research Assistant}

Aug 2006 Department of Education \& Counseling Psychology

University of Louisville, Louisville, KY

- Participated in ongoing research related but not limited to the achicvement gap, children's adjustment to parental divorce, character education, identity development, cultural adaptation and cultural psichology, and shyness and academic achievement in elementary school children

- Duties included conducting empirically based literature reviews; data collection; data analyses; evaluating psychometric properties of developed measures of adjustment; assisted in preparing, presenting, and publishing research articles/ manuscripts

- Supervised work study student aide in conducting literature reviews and doing data entry on SPSS

Aug 2000- Graduate Research Team Member

Aug 2001 Department of Psychology, University of Dayton, Dayton, OH

- Assisted with ongoing rescarch conducted by Dr. Lane Geddie studying children's memory and eye witness testimony

- Involved in pre-implementation planning of research project

\section{TEACHING EXPERIENCE}

Jan 2008- Instructor, Psychology Across Cultures

May 2008 Department of psychology, Miami University, Oxford, Ohio

- Responsibilities include designing course, lecturing, integrating multimedia, grading, and Blackboard

\section{OUTREACH \& PROGRAMMING EXPERIENCE}

Aug 2007 Student Counseling Service

Present Miami University, Oxford, Ohio

- New International Student Orientation 
- Diversity Advisory Counsel

- Introduction of Student Counseling Services to Incoming Freshman

- Pre-doctoral Internship Application Training

- Behind Closed Doors, Training for Residential Staff

- Acculturation \& School Development of Somali Bantu Children

- Eating Disorders \& Body Image Issues on College Campus

- Culture Shock and Criss Cultural Communication

\section{PRESENTATIONS \& PUBLICATIONS}

Rudasill, K. M., Mitchell, N. (;., White, J. B., \& Sckhon, M. (in progress). Contributions of children's temperament to social and academic outcomes in elementary school.

Rivera, S., Henry, C., l.i, Y., \& Sekhon, M., (September, 2007). Sociocultural and Clinical Challenges in the Pratice of Malticultural Therapy at University Connseling Centers. Presented at the Dennis H. May Conference on Diversity Issues \& the Role of Counseling Centers, Liniversity of Illinois, Urbana, IL.

Portes, P.R., Brown, I.H., Saylor, K., \& Sekhon, M., (20)5). Assessing children's adjustment to divorce stress: A validation of the Divorce Adjustment Inventory - Revised scales through family functioning and child adjustment standard measures. Journal of Divore e Remarriage, 44(1/2).

Cunningham, N. J., Hicks, J. Jook, J.., Parrish, M., \& Sekhon, M., (October, 2004). Building Peacmaking Skills in Youth: Youth Frorums for Peace. Presented at the 47th Annual Kentucky Counscling Association Conference. J ouisville, Kentucky.

Pemberton, C., \& Sckhon, M., (October, 2004). Pharmacological Intermentions for $1 D H D$. Presented at the 47th Annual Kentucky Counseling Association Conference. louisville, Kentucky.

Portes, P.R., \& Sckhon, M., (October, 2004). Dismanling the Acbievement Gap in Education. A Cuttural-I Hstorical Model for Eradicating Educational Inequality in the U.S. Poster presented at the 47th Annual Kentucky Counseling Association Conference. Louisville, Kentucky.

Cunningham, N. J., Look, L., \& Sekhon, M., (September, 2004). Yrouth liomms for Peace: Building Peacmaking Skills in Youth. Presented at the Post-Secondary Advisory Group Luncheon at the Kentucky Safe Schools Conference, Louisville, Kentucky.

Sekhon, M., (2003). Differences amongst Field Dependent \& Independent Individuals in their Reactions to Paintings. Master's Thesis, University of Dayton, Dayton, $\mathrm{OH}$. 


\section{PROFESSIONAL AFFILIATIONS}

2005-2007 Safè Harbor Refugee Treatment Program Committee, Louisville, KY

2005 - 2006 American Psychological Association of Graduate Students, Committee on Ethnic Minority Affairs, Regional Diversity Coordinator

$2005-2006 \quad$ Rotaract Club, University of Louisville

2004 - 2007 Kentucky Psychological Association Student Affiliate

2004 - Present American Psychological Association Student Affiliate

2005-2006 American Psychological Association of Graduate Students, Advocacy Coordination Team, Campus Representative

2005 - 2006 Louisville Suicide Prevention Coalition,

Formative Evaluation Team, University of Louisville

$2005-2006 \quad$ Muhammad Ali Institute for Peace and Justice, University of Louisville, Louisville, KY

2003-2004 Educational and Counseling Psychology Doctoral Student Organization, Program Chair

HONORS

$2006-2007$ University Fellowship, University of Louisville. Louisville, KY

2005 Kentucky Psychological Association Multicultural Professional Development Award

2002 Summer Graduate Fellowship, University of Dayton, Dayton, Ohio

2002 Koehler International Student Award, University of Dayton

$1992-1994 \quad$ National Merit Scholarship, Indian Air Force

\section{LANGUAGES}

Fluent in English, Hindi and Punjabi; 